\title{
Symplectic Leaves of Complex Reductive Poisson-Lie Groups
}

\author{
Milen Yakimov \\ Department of Mathematics, \\ University of California at Berkeley, \\ Berkeley, CA 94720, USA *
}

\begin{abstract}
All factorizable Lie bialgebra structures on complex reductive Lie algebras were described by Belavin and Drinfeld. We classify the symplectic leaves of the full class of corresponding connected Poisson-Lie groups. A formula for their dimensions is also proved.
\end{abstract}

\section{Introduction}

The structure of symplectic leaves of a Poisson manifold $M$ naturally carries information about the geometry of the Poisson bivector field. Its importance to dynamical systems is related to the fact that the phase space of any Hamiltonian system on $M$ is a symplectic leaf of $M$. When the Poisson structure can be quantized algebraically, it has also applications to the representation theory of the corresponding quantized algebra of functions. Roughly speaking the Poisson ideals of functions vanishing on closures of symplectic leaves are deformed to primitive ideals of the quantized algebra, but the actual relation is subtle. In the 70's and 80's a detailed study of the primitive ideals of universal enveloping algebras of Lie algebras and their relation to coadjoint orbits was done. Nice accounts to it can be found in the review article [16] and the book [4]. For standard (simple) Poisson-Lie groups and their quantized algebras of functions, this relation was examined more recently by Soibelman [26], Hodges-Levasseur [10, 11], and Joseph [17, 18] (see also [20, 12]).

In the early 80's Belavin and Drinfeld classified all nonskewsymmetric $r$-matrices on complex simple Lie algebras. The same procedure describes all factorizable Lie bialgebra structures on complex reductive Lie algebras, see [9]. The set of symplectic leaves on the corresponding Poisson-Lie groups is well understood for the standard structure [8, 10, 13], on the corresponding compact real forms [26, 21, 19], and in the cases of the simplest twistings of the standard $r$-matrix by elements of the second tensor power of the (fixed) Cartan subalgebra [20, 12]. In the same time very little is known for any other Poisson structure in this list.

The goal of this article is to present a unified description of the sets of symplectic leaves of the complex reductive Poisson-Lie groups associated to all $r$-matrices from BelavinDrinfeld's list. We also prove a formula for the dimension of each leaf.

\footnotetext{
*E-mail address: yakimov@math.berkeley.edu
} 
In the rest of this introduction we will give a rough description of our results. Any Poisson-Lie group $G$ of the discussed class has two canonical Poisson-Lie subgroups $G_{ \pm}$. Their Lie algebras are the spans of the second and the first components of the associated $r$-matrix. $G_{ \pm}$lie inside two parabolic subgroups $P_{ \pm}$of $G$ and differ from them only on a part of a maximal torus $H \subset P_{ \pm}$. Let $W, W_{1}$, and $W_{2}$ denote the Weyl groups of $G$ and the Levi factors $L_{1}, L_{2}$ of $P_{ \pm}$.

The main ingredient in the classification theorem for the leaves of $G_{-}$is a choice of minimal length representative $v$ in $W$ for a coset from $W / W_{1}$. (By $\dot{v}$ we will denote a representative of it in the normalizer $N(H)$ of $H$ in $G$.) For each such element $v$ the maximal subalgebra of $\operatorname{Lie}\left(L_{1}\right)$ which is stable under $\operatorname{Ad}_{\dot{v}}$ is a reductive subalgebra of $\mathfrak{g}$ generated by the Cartan subalgebra $\operatorname{Lie}(H)$ and root spaces of simple roots. The corresponding reductive subgroup of $G$ will be denoted by $G^{v}$. The second main ingredient in the classification is a choice (for each fixed $v$ ) of an orbit of the twisted conjugation action of the derived subgroup $\left(G^{v}\right)^{\prime}$ of $G^{v}$ on itself defined by

$$
T C_{g}^{\dot{v}}(f)=g^{-1} f \operatorname{Ad}_{\dot{v}}(g), f, g \in\left(G^{v}\right)^{\prime} .
$$

In Sect. 4.1 we prove that this is the main part of the data which classifies double cosets of the dual Poisson-Lie group $G_{+}$of $G_{-}$in their classical double. Sections 4.2 and 4.3 realize the passage to symplectic leaves (dressing orbits) on $G_{-}$. The set of symplectic leaves of $G_{+}$are described in a similar way, but the corresponding results will not be stated.

Sect. 5 deals with the general case of symplectic leaves on the full group $G$. The classification data is partly doubling of the one for $G_{-}$. One starts with a choice of a pair $\left(v_{1}, v_{2}\right)$ of minimal length representatives in $W$ for cosets from $W / W_{1}$ and $W / W_{2}$. The second part of the data is a twisted conjugation orbit on the maximal semisimple subgroup of $L_{1}$ whose Lie algebra is stable under $\operatorname{Ad}_{\dot{v}_{1}} \theta^{-1} \operatorname{Ad}_{\dot{v}_{2}} \theta$. The map $\theta: \operatorname{Lie}\left(L_{1}^{\prime}\right) \rightarrow \operatorname{Lie}\left(L_{2}^{\prime}\right)$ is an isomorphism known as Cayley transform of the $r$-matrix $r$.

The first three Sections contain both a review of known facts that we need and some new results, see in particular Sections 1.3, 2.2, 2.3, and 3.3.

For simplification purposes we have chosen to state and prove fully the results for the Poisson-Lie subgroup $G_{-}$of $G$. The ones for $G$ are stated, but their proofs are mostly sketched. Although they imply the ones for $G_{-}$, their proofs compared to the $G_{-}$case contain additional complications only.

Our proofs are based on a certain inductive procedure which resembles (and in some sense should be considered as Poisson-Lie analog of) Duflo's construction for computing the primitive spectrum of universal enveloping algebras of Lie algebras which are not necessarily solvable or semisimple [5]. The reason for this relation comes from the fact that linearization of the Poisson structure on $G$ at the origin and quantization produces the universal enveloping algebra $U\left(\mathfrak{g}^{*}\right)$ of the dual Lie bialgebra $\mathfrak{g}^{*}$ of $\mathfrak{g}=\operatorname{Lie}(G)$. The Lie algebra $\mathfrak{g}^{*}$ is roughly speaking isomorphic to a sum of two parabolic subalgebras of $\mathfrak{g}$ with identified Levi factors. It is solvable only in the cases of the simplest twistings of the standard structure.

An explicit quantization of the Belavin-Drinfeld $r$-matrices was obtained recently by Etingof, Schedler, and Schiffmann [6]. An appropriate extension of Duflo's construction will be essential for the classification the primitive ideals of the corresponding quantized algebras of functions. 
In a subsequent publication [27] we will extend our methods to classify the symplectic leaves of the Poisson homogeneous spaces for the considered groups.

A word on terminology and notation: All double cosets will be considered as orbits of actions of Lie groups and as such will be equipped with the induced quotient topology. All results will refer to this topology. Often there will be formulas that mix direct sums of linear spaces and direct sums of Lie algebras. To distinguish them, the former will be denoted by $\dot{+}$ and the latter by $\oplus$.

Acknowledgements I am grateful to Nicolai Reshetikhin for teaching me Poisson-Lie groups and for many stimulating discussions. I also benefited a lot from fruitful conversations with G. Ames, E. Frenkel, A. Givental, T. Hodges, T. Levasseur, A. Okounkov, A. Weinstein, and J. Wolf. This work was supported from the NSF grants DMS96-03239 and DMS94-00097.

\section{Lie bialgebras and Poisson-Lie groups}

All Lie algebras and groups will be assumed finite dimensional.

\subsection{Lie bialgebras}

Definition 1.1 A Lie bialgebra is a Lie algebra $(\mathfrak{g},[.,]$.$) equipped with a map \delta: \mathfrak{g} \rightarrow \wedge^{2} \mathfrak{g}$, called Lie cobracket, such that

1. The dual map to $\delta$ defines a Lie algebra structure on $\mathfrak{g}^{*}$ and

2. $\delta([a, b])=[\delta(a), 1 \otimes b+b \otimes 1]+[1 \otimes a+a \otimes 1, \delta(b)], \quad \forall a, b \in \mathfrak{g}$.

The notion of Lie bialgebra is self dual. More precisely, the dual space $\mathfrak{g}^{*}$ is naturally equipped with a structure of Lie bialgebra with Lie bracket induced by $\delta$ and Lie cobracket induced by $[.,$.$] . The same Lie algebra equipped with the opposite cobracket will be denoted$ by $\mathfrak{g}^{* o p}$.

Definition 1.2 The space $\mathfrak{g} \dot{+} \mathfrak{g}^{*}$ admits a canonical structure of Lie bialgebra called classical (Drinfeld) double of $\mathfrak{g}$ and denoted by $\mathrm{D}(\mathfrak{g})$. It is uniquely determined from the requirements:

1. The natural embeddings $i: \mathfrak{g} \hookrightarrow \mathrm{D}(\mathfrak{g})=\mathfrak{g} \dot{+} \mathfrak{g}^{*}$ and $i^{*}: \mathfrak{g}^{* \mathrm{op}} \hookrightarrow \mathrm{D}(\mathfrak{g})=\mathfrak{g} \dot{+} \mathfrak{g}^{*}$ are morphisms of Lie bialgebras.

2. The inner product on $\mathfrak{g} \dot{+} \mathfrak{g}^{*}$ which is equal to the pairing between $\mathfrak{g}$ and $\mathfrak{g}^{*}$ and is trivial otherwise, is invariant with respect to the adjoint action of $\mathrm{D}(\mathfrak{g})$.

The following is an important construction for studying the structure of a classical double.

Definition 1.3 A Manin triple is a triple of Lie algebras $\left(\mathfrak{g}, \mathfrak{a}_{+}, \mathfrak{a}_{-}\right)$for which $\mathfrak{g}$ is equipped with an invariant nondegenerate inner product (.,.) and

1. $\mathfrak{a}_{+}$and $\mathfrak{a}_{-}$are isotropic Lie subalgebras of $\mathfrak{g}$,

2. $\mathfrak{g}=\mathfrak{a}_{+} \dot{+} \mathfrak{a}_{-}$. 
The double $\mathrm{D}(\mathfrak{g})$ of any Lie bialgebra $\mathfrak{g}$ gives rise to a Manin triple $\left(\mathrm{D}(\mathfrak{g}), \mathfrak{g}, \mathfrak{g}^{*}\right)$ with inner product on $\mathfrak{g} \dot{+} \mathfrak{g}^{*}$ as defined in Definition 1.2. Conversely, assume that $\left(\mathfrak{g}, \mathfrak{a}_{+}, \mathfrak{a}_{-}\right)$ is a Manin triple. Then $\mathfrak{a}_{+}, \mathfrak{a}_{-}$, and $\mathfrak{g}$ have natural structures of Lie bialgebras with

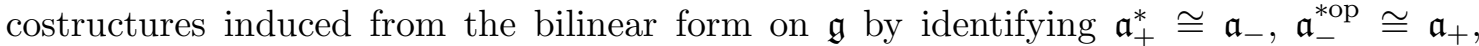
and setting $\mathfrak{g}=\mathfrak{a}_{+} \oplus \mathfrak{a}_{-}$as Lie coalgebras. One finds that as bialgebras $\mathfrak{a}_{-} \cong \mathfrak{a}_{+}^{* o p}$ and $\mathfrak{g} \cong \mathrm{D}\left(\mathfrak{a}_{+}\right)$.

Definition 1.4 A Lie bialgebra $(\mathfrak{g},[.,],. \delta)$ is called coboundary if there exists an element $r \in \mathfrak{g} \otimes \mathfrak{g}$ such that

$$
\delta(a)=[r, a \otimes 1+1 \otimes a], \quad \forall a \in \mathfrak{g} .
$$

The map $\delta$ from (1.1) defines a Lie bialgebra structure on $\mathfrak{g}$ if and only if the following conditions hold for $r$ :

$$
\begin{aligned}
& r+r_{21} \in\left(\mathfrak{g}^{\otimes 2}\right)^{\mathfrak{g}}, \\
& {\left[r_{12}, r_{13}\right]+\left[r_{12}, r_{23}\right]+\left[r_{13}, r_{23}\right] \in\left(\mathfrak{g}^{\otimes 3}\right)^{\mathfrak{g}} .}
\end{aligned}
$$

For the standard notation $r_{12}, r_{13}$, etc., we refer to any book on quantum groups, e.g. [2, 7, 20]. In both formulas $(.)^{\mathfrak{g}}$ means $\mathfrak{g}$-invariant part with respect to the natural adjoint action of $\mathfrak{g}$ on tensor powers of $\mathfrak{g}$.

Definition 1.5 A coboundary Lie bialgebra is called quasitriangular if its $r$-matrix satisfies the Yang-Baxter equation

$$
\left[r_{12}, r_{13}\right]+\left[r_{12}, r_{23}\right]+\left[r_{13}, r_{23}\right]=0
$$

The classical double $\mathrm{D}(\mathfrak{g})$ of any Lie bialgebra $\mathfrak{g}$ is a quasitriangular bialgebra with $r$ matrix $r \in \mathfrak{g} \otimes \mathfrak{g}^{*}$ representing the graph of the trivial map id $: \mathfrak{g} \rightarrow \mathfrak{g}$.

For any quasitriangular Lie bialgebra $\mathfrak{g}$ one defines the maps $r_{+}$and $r_{-}: \mathfrak{g}^{*} \rightarrow \mathfrak{g}$ by

$$
\begin{aligned}
& r_{+}(x)=(x \otimes \mathrm{id}) r, \quad \forall x \in \mathfrak{g}^{*}, \\
& r_{-}(x)=-(\mathrm{id} \otimes x) r, \quad \forall x \in \mathfrak{g}^{*} .
\end{aligned}
$$

Lemma 1.6 The maps $r_{+}, r_{-}: \mathfrak{g}^{*} \rightarrow \mathfrak{g}$ are morphisms of Lie bialgebras.

See [23, 22, [] for a proof of this Lemma. Denote the images of $r_{+}$and $r_{-}$by $\mathfrak{g}_{+}$and $\mathfrak{g}_{-}$ respectively.

Definition 1.7 A quasitriangular Lie bialgebra $\mathfrak{g}$ is called factorizable 23] if

$$
r+r_{21} \in S^{2} \mathfrak{g}
$$

defines a nondegenerate inner product on $\mathfrak{g}^{*}$. (Here $S^{k} V$ denotes the $k$-th symmetric power of the vector space $V$.) 
For any quasitriangular Lie bialgebra $\mathfrak{g}$, the spaces $\mathfrak{g}_{+}$and $\mathfrak{g}_{-}$are Lie sub-bialgebras of $\mathfrak{g}$ and $\mathfrak{g}_{-} \cong \mathfrak{g}_{+}^{* o p}$. Thus $\mathrm{D}\left(\mathfrak{g}_{+}\right)$as a vector space is isomorphic to $\mathfrak{g}_{+} \dot{+} \mathfrak{g}_{-}$and one can define a linear map

$$
\pi: \mathrm{D}\left(\mathfrak{g}_{+}\right) \rightarrow \mathfrak{g}
$$

which restricted to $\mathfrak{g}_{+}$and $\mathfrak{g}_{-}$coincides with the natural embeddings of these algebras in g.

Lemma 1.8 The map $\pi$ defined in (1.7) is a morphism of Lie bialgebras.

Proofs of this Lemma can be found in [23, 22, 7].

\subsection{Symplectic leaves of Poisson-Lie groups}

Recall that a symplectic leaf of a Poisson manifold $(M, \pi)$ is a maximal connected symplectic submanifold. Any symplectic leaf $S$ can also be described as a union of all piecewise smooth paths starting at a given point of $S$ with segments that are integral curves of Hamiltonian vector fields on $M$. In particular this implies that $M$ is a disjoint union of its symplectic leaves.

A Poisson-Lie group $G$ is a Lie group equipped with a Poisson bivector field for which the multiplication map $G \times G \rightarrow G$ is a Poisson map. Its tangent Lie algebra $\mathfrak{g}$ carries a natural Lie bialgebra structure and conversely for any Lie bialgebra $\mathfrak{g}$ there exists a unique connected and simply connected Poisson-Lie group whose tangent Lie bialgebra is $\mathfrak{g}$ (see [2]). Any Poisson-Lie group $G$ with coboundary tangent Lie bialgebra $\mathfrak{g}$ can be equipped with the Sklyanin Poisson-Lie structure

$$
\pi_{x}=L_{x}(r)-R_{x}(r), x \in G
$$

where $r \in \mathfrak{g} \otimes \mathfrak{g} \equiv T_{e} G \otimes T_{e} G$ is an $r$-matrix for the Lie bialgebra structure on $\mathfrak{g}$ and $L_{x}$, $R_{x}$ denote the differentials of the right / left translations by $x \in G$. The bivector field $\pi$ is skewsymmetric because of the Ad-invariance of $r+r_{21}$, see (1.2).

A double $\mathrm{D}(G)$ of a Poisson-Lie group $G$ is a connected Lie group with $\operatorname{Lie}(\mathrm{D}(G))=$ $\mathrm{D}(\mathfrak{g})$ for which the embedding $i: \mathfrak{g} \hookrightarrow \mathrm{D}(\mathfrak{g})$ can be integrated to a (possibly not proper) embedding of Lie groups:

$$
i: G \rightarrow \mathrm{D}(G)
$$

Since $\mathrm{D}(\mathfrak{g})$ is a quasitriangular Lie bialgebra, any such group $\mathrm{D}(G)$ can be equipped with the Sklyanin Poisson structure and then $i$ becomes an embedding of Poisson-Lie groups. By $G^{r}$ we will denote the (generally not closed) subgroup of $\mathrm{D}(G)$ with $\operatorname{Lie}\left(G^{r}\right)=i^{*}\left(\mathfrak{g}^{*}\right) \subset$ $\mathrm{D}(\mathfrak{g}) . G^{r}$ will be equipped with the topology having as a basis of open sets the connected components of intersections of open sets of $\mathrm{D}(G)$ with $G^{r}$. Since $i^{*}\left(\mathfrak{g}^{* \mathrm{op}}\right)$ is a Lie subbialgebra of $\mathrm{D}(\mathfrak{g}), G^{r}$ is a (nonproperly embedded) Poisson-Lie subgroup of $\mathrm{D}(G)$ with tangent Lie bialgebra $i^{*}\left(\mathfrak{g}^{* \mathrm{op}}\right) \cong \mathfrak{g}^{* \mathrm{op}}$. It naturally plays the role of (opposite) dual of $G$.

The intersection

$$
\Sigma:=i(G) \cap G^{r}
$$

is a discrete subgroup of $\mathrm{D}(G)$ because $i(\mathfrak{g}) \cap i^{*}\left(\mathfrak{g}^{*}\right)=0$. The right action of $\Sigma$ on $i(G)$ preserves the Poisson bivector field. By abuse of notation the projections of the symplectic leaves of $G$ on $i(G) / \Sigma$ will be called symplectic leaves of $i(G) / \Sigma$. In the case when $i(G)$ and 
$G^{r}$ are closed subgroups of $\mathrm{D}(G)$, the latter are indeed the symplectic leaves of $i(G) / \Sigma$. In this case $i(G) / \Sigma$ is also diffeomorphic as a Poisson manifold to the dense, open subset $i(G) G^{r} / G^{r}$ of the Poisson homogeneous space $\mathrm{D}(G) / G^{r}$ of $\mathrm{D}(G)$. The projection map $G \rightarrow i(G) / \Sigma$ will be denoted by $p$.

In this setting an algebraic way to describe the symplectic leaves of $G$ was found by Semenov-Tian-Shansky [25] (see also [21]). In this formulation the statement was taken from [10].

Theorem 1.9 1. The symplectic leaves of $i(G) / \Sigma$ are the connected components of the intersections of double cosets $G^{r} x G^{r}$ in $\mathrm{D}(G)$ with $i(G) G^{r}$ projected into $i(G) G^{r} / G^{r} \cong$ $i(G) / \Sigma$.

2. The symplectic leaves of $G$ are connected components of the pull backs of symplectic leaves of $i(G) / \Sigma$ under the projection $p$.

In the case when $i(G)$ and $G^{r}$ are closed subgroups of $\mathrm{D}(G)$, the left (local) action of $G^{r}$ on $i(G) G^{r} / G^{r}$ can be lifted to a (local) Poisson action of $G^{r}$ on $G$ using the the covering map $p$. In the general case such an action is obtained by integrating the so called dressing vector fields, see [21]. This action is called left dressing action of the (opposite) dual Poisson-Lie group $G^{r}$ of $G$, on $G$. The traditional way of stating the result of Theorem 1.9 is that the symplectic leaves of $G$ are the orbits of the dressing action of $G^{r}$.

\subsection{The passage from double cosets to symplectic leaves}

According to Theorem 1.9 the main question in passing from $G^{r}$ double cosets in $\mathrm{D}(G)$ to symplectic leaves of $i(G) / \Sigma$ is to understand whether (and how) each such double coset intersects the dense, open subset $i(G) G^{r}$ of $\mathrm{D}(G)$. In this Subsection we provide an answer to this question for an arbitrary Poisson-Lie group.

Theorem 1.10 For any connected complex Poisson-Lie group $G$ the intersection of each double coset $G^{r} x G^{r}$ in $\mathrm{D}(G)$ with $i(G) G^{r}$ is a dense, open, and connected subset of the coset $G^{r} x G^{r}$.

Taking into account $i(\mathfrak{g}) \dot{+} i^{*}\left(\mathfrak{g}^{*}\right)=\mathrm{D}(\mathfrak{g})$, Theorem 1.10 can be obtained as a consequence from the following Lemma.

Lemma 1.11 Let $C$ be a connected complex Lie group and $A, B$ be two Lie subgroups of C satisfying

$$
\operatorname{Lie}(A) \dot{+} \operatorname{Lie}(B)=\operatorname{Lie}(C) .
$$

Then the intersection $A x A \cap B A$ is a dense, open, and connected subset of $A x A$ for any $x \in C$.

Proof. Eq. (1.9) implies that the products $A B$ and $B A$ are dense, open subsets of $C$. Recall that if $U$ and $V$ are two dense, open subsets of $C$, then $U V=C$ (see e.g. Lemma 7.4 in [14]). Applying this for $U=A B$ and $V=B A$ shows

$$
A B A=C .
$$

Thus the intersection $A x A \cap B A$ is a nonempty open subset of $A x A$, for all $x \in C$. 
$B A$ can be considered as the orbit of $e \in C$ under the obvious action of $A \times B$. Consider the general situation of an action of a Lie group $F$ on a complex manifold $X$ for which there is a dense, open orbit $\mathcal{O}$ of dimension $\operatorname{dim} F$. We will construct a global section of the anticanonical bundle $K_{X}^{*}$ on $X$ whose set of zeros is the complement to $\mathcal{O}$. Fix a linear basis $\left\{f_{k}\right\}_{k=1}^{N}$ of $\operatorname{Lie}(F), N=\operatorname{dim} F$. The infinitesimal action of $\operatorname{Lie}(F)$ gives rise to a set of vector fields $\left\{v_{k}\right\}_{k=1}^{N}$ on $X$. The assumption on the action of $F$ implies that the set of vectors $\left\{v_{1}(x), \ldots, v_{N}(x)\right\} \subset T_{x} X$ is linearly independent for $x \in \mathcal{O}$ and linearly dependent otherwise. Thus the global section $v_{1} \wedge \ldots \wedge v_{N}$ of the anticanonical bundle on $X$ vanishes exactly on the complement to the orbit $\mathcal{O}$ in $X$. Applying this argument to the above mentioned action of $A \times B$ on $C$ gives that $A x A \cap B A$ is a nonempty open subset of $B A$ whose complement in $B A$ is the zero set of a global section of $K_{C}^{*}$. This implies that it is a dense, open subset of $B A$ which in addition is connected since the ground field is $\mathbb{C}$.

Note also that Lemma 1.11 without the connectedness part is still valid over $\mathbb{R}$.

\section{Lie algebra structure of certain doubles and duals}

This Section is devoted to the explicit description of the Lie algebra structure of certain doubles and duals associated to a factorizable Lie bialgebra $\mathfrak{g}$. (In all statements the costructure of the involved bialgebras will not be considered.) First we recall a result of Reshetikhin and Semenov-Tian-Shansky [23] on Lie algebra structure of $\mathrm{D}(\mathfrak{g})$ and the embeddings of $\mathfrak{g}$ and $\mathfrak{g}^{*}$ in it. From it we deduce a similar result about the Lie structure of $\mathrm{D}\left(\mathfrak{g}_{+}\right)$and the embeddings of $\mathfrak{g}_{+}$and $\mathfrak{g}_{-}$. A second derivation is also presented. Compared to the first one, it has the the advantage that it does not rely on an initial guess of what the structure is.

The nondegenerate bilinear form on $\mathfrak{g}$ induced by $r+r_{21} \in S^{2} \mathfrak{g}$ will be used often and will be denoted by $(., .)_{r}$.

\subsection{Description of $D(\mathfrak{g})$ and of the embeddings $\mathfrak{g}, \mathfrak{g}^{*} \subset D(\mathfrak{g})$}

The homomorphisms $r_{ \pm}$(see eqs. (1.5)-(1.6) ) can be combined to define an embedding of Lie algebras

$$
r: \mathfrak{g}^{*} \rightarrow \mathfrak{g} \oplus \mathfrak{g}
$$

by

$$
r(x)=\left(r_{+}(x), r_{-}(x)\right), x \in \mathfrak{g}^{*} .
$$

This map is injective due to the nondegeneracy of $r+r_{21}$. Its image will be denoted by $\mathfrak{g}^{r} \cong \mathfrak{g}^{*}$.

The Lie algebra $\mathfrak{g}$ by itself can be embedded diagonally in $\mathfrak{g} \oplus \mathfrak{g}$ :

$$
d(g)=(g, g) \in \mathfrak{g} \oplus \mathfrak{g}, \text { for } g \in \mathfrak{g},
$$

so $d(\mathfrak{g}):=\operatorname{Im} d \cong \mathfrak{g}$.

Proposition 2.1 (Reshetikhin-Semenov-Tian-Shansky) The double $\mathrm{D}(\mathfrak{g})$ of a factorizable Lie bialgebra $\mathfrak{g}$ is isomorphic as a Lie algebra to $\mathfrak{g} \oplus \mathfrak{g}$. In addition $\mathfrak{g}^{*}$ and $\mathfrak{g}$ are embedded in it by the maps $r$ and d defined in (2.2) and (2.3). 
Proof. Equip $\mathfrak{g} \oplus \mathfrak{g}$ with the bilinear form

$$
<\left(x_{1}, x_{2}\right),\left(y_{1}, y_{2}\right)>=\left(x_{1}, y_{1}\right)_{r}-\left(x_{2}, y_{2}\right)_{r} .
$$

One checks that $d(\mathfrak{g})$ and $\mathfrak{g}^{r}$ are isotropic subspaces of $\mathfrak{g}$. Since $d(\mathfrak{g}) \cap \mathfrak{g}^{r}=0$ and $\operatorname{dim} d(\mathfrak{g})+\operatorname{dim} \mathfrak{g}^{r}=2 \operatorname{dim} \mathfrak{g}$ the triple $\left(\mathfrak{g} \oplus \mathfrak{g}, d(\mathfrak{g}), \mathfrak{g}^{r}\right)$ is a Manin triple, which implies the statement.

The algebra $\mathfrak{g}^{r}$ can be described more explicitly in terms of the so called Cayley transform of $r$. To formulate this result, we need to introduce the algebras

$$
\begin{aligned}
& \mathfrak{m}_{+}=r_{+}\left(\operatorname{Ker} r_{-}\right) \\
& \mathfrak{m}_{-}=r_{-}\left(\operatorname{Ker} r_{+}\right)
\end{aligned}
$$

Clearly $\mathfrak{m}_{ \pm}$are Lie ideals of $\mathfrak{g}_{ \pm}$. The quotients $\mathfrak{g}_{+} / \mathfrak{m}_{+}$and $\mathfrak{g}_{-} / \mathfrak{m}_{-}$are isomorphic as Lie algebras and the isomorphism is given by

$$
\theta\left(r_{+}(x)+\mathfrak{m}_{+}\right)=r_{-}(x)+\mathfrak{m}_{-}, \forall x \in \mathfrak{g}^{*} .
$$

It is correctly defined, since $r_{+}(x) \in \mathfrak{m}_{+}$implies $x \in \operatorname{Ker} r_{+}+\operatorname{Ker} r_{-}$and thus $r_{-}(x) \in \mathfrak{m}_{-}$. It is also straightforward to check that $\theta$ is a Lie algebra homomorphism. The fact that it is an isomorphism is proved directly by constructing the inverse map $\theta^{-1}: \mathfrak{g}_{-} / \mathfrak{m}_{-} \rightarrow \mathfrak{g}_{+} / \mathfrak{m}_{+}$ by

$$
\theta\left(r_{-}(x)+\mathfrak{m}_{-}\right)=r_{+}(x)+\mathfrak{m}_{+}, \forall x \in \mathfrak{g}^{*} .
$$

The map $\theta$ is the classical Cayley transform " $\frac{f}{f-1}$ " of the linear map $f: \mathfrak{g} \rightarrow \mathfrak{g}$, defined by

$$
f=-r_{-} \circ j: \mathfrak{g} \rightarrow \mathfrak{g}
$$

where $j: \mathfrak{g} \rightarrow \mathfrak{g}^{*}$ is the linear isomorphism associated with the nondegenerate form $(., .)_{r}$. In terms of it: $r_{+} \circ j=1-f$. The algebras $\mathfrak{g}_{ \pm}, \mathfrak{m}_{ \pm}$, are $\mathfrak{g}_{+}=\operatorname{Im}(f-1), \mathfrak{g}_{-}=\operatorname{Im} f$, $\mathfrak{m}_{+}=\operatorname{Ker} f, \mathfrak{m}_{-}=\operatorname{Ker}(f-1)$ as needed for the definition of Cayley transform.

Consider also the projections:

$$
\mathrm{p}_{ \pm}: \mathfrak{g}_{ \pm} \rightarrow \mathfrak{g}_{ \pm} / \mathfrak{m}_{ \pm}
$$

The following result of Semenov-Tian-Shansky [24] describes $\mathfrak{g}^{r}$ in terms of $\theta$.

Proposition 2.2 The subalgebra $\mathfrak{g}^{r}$ of $\mathfrak{g}_{+} \oplus \mathfrak{g}_{-} \subset \mathfrak{g} \oplus \mathfrak{g}$ consists of those $\left(x_{+}, x_{-}\right) \in \mathfrak{g}_{+} \oplus \mathfrak{g}_{-}$ for which

$$
\theta \circ \mathrm{p}_{+}\left(x_{+}\right)=\mathrm{p}_{-}\left(x_{-}\right) \text {. }
$$

This proposition is obtained directly from the definition of $\theta$.

Note that $\mathfrak{m}_{ \pm}$are isotropic subalgebras of $\mathfrak{g}_{ \pm}$with respect to the bilinear form $(., .)_{r}$. Indeed, for all $x \in \operatorname{Ker} r_{-} \subset \mathfrak{g}^{*}$ and $y \in \mathfrak{g}^{*}$ we have

$$
\begin{aligned}
& \left(r_{+}(x), r_{+}(y)\right)_{r}=x\left(r_{+}(y)\right) \\
& =(y \oplus x) r=-y\left(r_{-}(x)\right)=0 .
\end{aligned}
$$

Therefore one can equip $\mathfrak{g}_{+} / \mathfrak{m}_{+}$and $\mathfrak{g}_{-} / \mathfrak{m}_{-}$with inner products induced by $(., .)_{r}$. In this setting the map $\theta$ is an isometry. 


\subsection{Description of $\mathrm{D}\left(\mathfrak{g}_{+}\right)$and of the embeddings $\mathfrak{g}_{+}, \mathfrak{g}_{-} \rightarrow \mathrm{D}\left(\mathfrak{g}_{+}\right)$}

Here we will describe the Lie structure of $\mathrm{D}\left(\mathfrak{g}_{+}\right)$and how $\mathfrak{g}_{+}$and $\mathfrak{g}_{-}$are embedded in it.

Proposition 2.3 As a Lie algebra

$$
\mathrm{D}\left(\mathfrak{g}_{+}\right) \cong \mathfrak{g} \oplus\left(\mathfrak{g}_{+} / \mathfrak{m}_{+}\right)
$$

The embeddings

$$
i_{ \pm}: \mathfrak{g}_{ \pm} \rightarrow \mathrm{D}\left(\mathfrak{g}_{+}\right) \cong \mathfrak{g} \oplus\left(\mathfrak{g}_{+} / \mathfrak{m}_{+}\right)
$$

are given by the formulas

$$
\begin{array}{cc}
i_{+}(x)=\left(x, \mathrm{p}_{+}(x)\right), & \text { for } x \in \mathfrak{g}_{+}, \\
i_{-}(y)=\left(y, \theta^{-1} \circ \mathrm{p}_{-}(y)\right), & \text { for } y \in \mathfrak{g}_{-} .
\end{array}
$$

Proof. Equip the Lie algebra $\mathfrak{g} \oplus\left(\mathfrak{g}_{+} / \mathfrak{m}_{+}\right)$with the invariant bilinear form

$$
<\left(x_{1}, x_{2}\right),\left(y_{1}, y_{2}\right)>=\left(x_{1}, y_{1}\right)_{r}-\left(x_{2}, y_{2}\right)_{r}, x_{1}, y_{1} \in \mathfrak{g}, x_{2}, y_{2} \in \mathfrak{g}_{+} / \mathfrak{m}_{+} .
$$

As was noticed, this is possible since $\mathfrak{m}_{+}$is an isotropic subspace of $\mathfrak{g}_{+}$with respect to the bilinear form $(., .)_{r}$. Eqs. (2.9)-2.10) define embeddings of $\mathfrak{g}_{ \pm}$in $\mathfrak{g} \oplus\left(\mathfrak{g}_{+} / \mathfrak{m}_{+}\right)$. Their images $i_{ \pm}\left(\mathfrak{g}_{ \pm}\right)$are isotropic subspaces with trivial intersection. This follows from

$$
\begin{aligned}
& i_{+}\left(\mathfrak{g}_{+}\right)=\left(\mathrm{id} \times \mathrm{p}_{+}\right) d\left(\mathfrak{g}_{+}\right), \\
& i_{-}\left(\mathfrak{g}_{-}\right)=\left(\mathrm{p}_{+} \times \mathrm{id}\right)\left(\mathfrak{g}^{r} \cap\left(\mathfrak{g}_{+} \oplus \mathfrak{g}\right)\right),
\end{aligned}
$$

and the fact that $d(\mathfrak{g})$ and $\mathfrak{g}^{r}$ are isotropic subspaces of $\mathfrak{g} \oplus \mathfrak{g}$ with respect to the inner product (2.4). In the second equation the elements of $\mathfrak{g} \oplus\left(\mathfrak{g}_{+} / \mathfrak{m}_{+}\right)$in the right hand side are written as pairs in the opposite order. Taking into account $\operatorname{dim} \mathfrak{g}_{+}+\operatorname{dim} \mathfrak{m}_{+}=\operatorname{dim} \mathfrak{g}$ one gets that $\left(\mathfrak{g} \oplus\left(\mathfrak{g}_{+} / \mathfrak{m}_{+}\right), i_{+}\left(\mathfrak{g}_{+}\right), i_{-}\left(\mathfrak{g}_{-}\right)\right)$is a Manin triple which implies the statement.

\subsection{Another approach to the structure of $\mathrm{D}\left(\mathfrak{g}_{+}\right)$}

Here we will present a second approach to the results from Sect. 2.2 on the Lie structure of $\mathrm{D}\left(\mathfrak{g}_{+}\right)$. All results will be formulated without proofs.

The starting point is the Lie bialgebra morphism $\pi: \mathrm{D}\left(\mathfrak{g}_{+}\right) \rightarrow \mathfrak{g}$, see (1.7). Its kernel

$$
\mathrm{Cg}:=\left\{(x,-x) \mid x \in \mathfrak{g}_{+} \cap \mathfrak{g}_{-}\right\} \subset \mathfrak{g}_{+} \dot{+} \mathfrak{g}_{-}
$$

is a Lie bialgebra ideal of $\mathrm{D}(\mathfrak{g})$. Define a map

$$
I: \mathfrak{g} \rightarrow \mathrm{D}\left(\mathfrak{g}_{+}\right)
$$

by

$$
I(x)=\left(r_{+} \circ j(x),-r_{-} \circ j(x)\right) \in \mathfrak{g}_{+} \dot{+} \mathfrak{g}_{-} \cong \mathrm{D}\left(\mathfrak{g}_{+}\right)
$$

where as before $j: \mathfrak{g} \rightarrow \mathfrak{g}^{*}$ is the identification map coming from the nondegenerate bilinear form $(., .)_{r}$ on $\mathfrak{g}$. 
Lemma 2.4 $I$ is an injective homomorphism of Lie algebras, such that $r \circ I=\mathrm{id}_{\mathfrak{g}}$.

This proposition allows us to split $\mathrm{D}\left(\mathfrak{g}_{+}\right)$as a direct sum of Lie algebras

$$
\mathrm{D}\left(\mathfrak{g}_{+}\right)=\operatorname{Im} I \oplus \mathrm{Cg} \cong \mathfrak{g} \oplus \mathrm{Cg} .
$$

To investigate the Lie structure of $\mathrm{Cg}$, we define the maps

$$
F_{ \pm}: \mathfrak{g}_{ \pm} / \mathfrak{m}_{ \pm} \rightarrow \mathrm{Cg} \subset \mathrm{D}\left(\mathfrak{g}_{+}\right)
$$

by

$$
\begin{aligned}
& F_{+}(x)=(f x,-f x) \in \mathrm{Cg}, \quad \forall x \in \operatorname{Im}(f-1), \\
& F_{-}(x)=((f-1) x,-(f-1) x) \in \mathrm{Cg}, \quad \forall x \in \operatorname{Im} f .
\end{aligned}
$$

They are clearly well defined on the factors.

Lemma 2.5 The maps $F_{+}$and $F_{-}$are Lie algebra isomorphisms and the composition

$$
\left(F_{-}\right)^{-1} \circ F_{+}: \mathfrak{g}_{+} / \mathfrak{m}_{+} \rightarrow \mathfrak{g}_{-} / \mathfrak{m}_{-}
$$

is equal to the Cayley transform $\theta: \mathfrak{g}_{+} / \mathfrak{m}_{+} \rightarrow \mathfrak{g}_{-} / \mathfrak{m}_{-}$of $f$.

Combining (2.15) with this Proposition implies the isomorphism (2.8). The statement about the inclusions $i_{ \pm}$of $\mathfrak{g}_{ \pm}$in $\mathrm{D}\left(\mathfrak{g}_{+}\right)$from Proposition 2.3 is obtained by computing the composition of the embeddings $\mathfrak{g}_{ \pm} \hookrightarrow \mathrm{D}\left(\mathfrak{g}_{+}\right)$with this isomorphism.

Finally note that when $\mathrm{D}\left(\mathfrak{g}_{+}\right)$is identified with $\mathfrak{g} \oplus\left(\mathfrak{g}_{+} / \mathfrak{m}_{+}\right)$, the projection $\pi: \mathrm{D}\left(\mathfrak{g}_{+}\right) \rightarrow \mathfrak{g}($ see $(1.7))$ is just the projection on the first component.

\section{Factorizable reductive Lie bialgebras and Poisson-Lie groups}

\subsection{Belavin-Drinfeld classification}

Consider a complex reductive Lie algebra $\mathfrak{g}$. Let $\mathfrak{h} \subset \mathfrak{g}$ be a Cartan subalgebra of $\mathfrak{g}$. Choose a set of positive roots $\Delta_{+}$and denote by $\mathfrak{b}_{ \pm}$the corresponding positive and negative Borel subalgebras of $\mathfrak{g}$. Let $\Gamma$ be the set of simple roots. For a fixed nondegenerate invariant inner product $(.,$.$) on \mathfrak{g},\left\{x_{\beta} \mid \beta \in \pm \Delta_{+}\right\}$will denote a set of root vectors $x_{\beta} \in \mathfrak{g}^{\beta}$, such that

$$
\left(x_{\beta}, x_{-\beta}\right)=1, \forall \beta \in \Delta_{+} .
$$

Definition 3.1 A Belavin-Drinfeld triple is a triple $\left(\Gamma_{1}, \Gamma_{2}, \tau\right)$ where $\Gamma_{1}, \Gamma_{2} \subset \Gamma$ and $\tau: \Gamma_{1} \rightarrow \Gamma_{2}$ is such that

1. $(\tau(\alpha), \tau(\beta))=(\alpha, \beta)$ for all simple roots $\alpha$ and $\beta$ from the set $\Gamma_{1}$,

2. For any $\gamma \in \Gamma_{1}$ there exists a positive integer $n$ for which $\tau^{n}(\gamma) \in \Gamma_{2} \backslash \Gamma_{1}$ (nilpotency condition).

Given a Belavin-Drinfeld triple $\left(\Gamma_{1}, \Gamma_{2} \subset \Gamma\right)$, one defines a partial ordering on $\Delta_{+}$by $\alpha<\beta$ if $\alpha \in \Gamma_{1}, \beta \in \Gamma_{2}$, and $\beta=\tau^{n}(\alpha)$ for an integer $n$. 
Theorem 3.2 (Belavin-Drinfeld) Any factorizable Lie bialgebra structure on a complex reductive Lie algebra $\mathfrak{g}$ has an $r$-matrix

$$
r=r_{0}+\sum_{\alpha \in \Delta_{+}} x_{-\alpha} \otimes x_{\alpha}+\sum_{\alpha, \beta \in \Delta_{+}, \alpha<\beta} x_{-\alpha} \wedge x_{\beta}
$$

for an appropriate choice of a Cartan subalgebra $\mathfrak{h}$, an invariant bilinear form (.,.) on $\mathfrak{g}$, root vectors $x_{\beta} \in \mathfrak{g}^{\beta}$ normalized by (3.1), and a Belavin-Drinfeld triple $\left(\Gamma_{1}, \Gamma_{2}, \tau\right)$. The component $r_{0} \in \mathfrak{h}^{\otimes 2}$ of $r$ has to be such that

$$
\begin{aligned}
& (\tau \alpha \otimes 1) r_{0}+(1 \otimes \alpha) r_{0}=0, \forall \alpha \in \Gamma_{1}, \\
& r_{0}+r_{0}^{21}=\Omega_{0} .
\end{aligned}
$$

Here $\Omega_{0}$ is the component in $\mathfrak{h} \otimes \mathfrak{h}$ of the Casimir of $\mathfrak{g}$ associated to the form (.,.).

For such an $r$-matrix the bilinear form $(., .)_{r}$ on $\mathfrak{g}$ induced by $r+r_{21}$ (see Sect. 2 ) is equal to the form $(.,$.$) .$

The original result of Belavin and Drinfeld dealt with the case of nonskewsymmetric $r$-matrices on complex simple Lie algebras. It was later observed (see [9]) that their proof can be easily extended to the above generality.

It is also important to note that all Lie bialgebra structures on a complex semisimple algebra $\mathfrak{g}$ are quasitriangular since $H^{2}(\mathfrak{g}, \mathfrak{g})=0$. But this is not true for a reductive algebra. For example when $\mathfrak{g}$ is commutative any Lie algebra structure on $\mathfrak{g}^{*}$ gives rise to Lie bialgebra structure on $\mathfrak{g}$.

Let us fix an $r$-matrix as in Theorem 3.2 associated to a Belavin-Drinfeld triple $\left(\Gamma_{1}, \Gamma_{2}, \tau\right)$. Denote with $\mathfrak{p}_{+}$the parabolic subalgebra of $\mathfrak{g}$ containing $\mathfrak{b}_{+}$and the root spaces $\mathfrak{g}^{\alpha}$ for all negative roots $\alpha$ that are linear combinations of simple roots from $\Gamma_{1}$. In a similar way define a parabolic subalgebra $\mathfrak{p}_{-}$containing $\mathfrak{b}_{-}$and $\mathfrak{g}^{\beta}$ for all positive roots $\beta$ that are linear combinations of simple roots from $\Gamma_{2}$.

With $\mathfrak{n}_{+}, \mathfrak{n}_{-}$we will denote the unipotent radicals of $\mathfrak{p}_{+}, \mathfrak{p}_{-}$and with $\mathfrak{l}_{1}, \mathfrak{l}_{2}$ their Levi factors.

The Levi decompositions of $\mathfrak{p}_{ \pm}$read:

$$
\mathfrak{p}_{ \pm}=\mathfrak{l}_{1,2} \dot{+} \mathfrak{n}_{ \pm}
$$

The derived subalgebras of $\mathfrak{l}_{1}, \mathfrak{l}_{2}$ will be denoted by $\mathfrak{l}_{1}^{\prime}, \mathfrak{l}_{2}^{\prime}$ and their centers by $\mathfrak{z}_{1}, \mathfrak{z}_{2}$, so

$$
\mathfrak{l}_{i}=\mathfrak{l}_{i}^{\prime} \oplus \mathfrak{z}_{i}, i=1,2 .
$$

The spans of first and second components of $r_{0}$ contain $\mathfrak{l}_{1}^{\prime} \cap \mathfrak{h}$ and $\mathfrak{l}_{2}^{\prime} \cap \mathfrak{h}$ respectively. Denote by $\mathfrak{h}_{1}$ and $\mathfrak{h}_{2}$ their intersections with $\mathfrak{z}_{1}$ and $\mathfrak{z}_{2}$. Finally define

$$
\mathfrak{h}_{i}^{\text {ort }}=\left[\mathfrak{h}_{i} \oplus\left(\mathfrak{l}_{i}^{\prime} \cap \mathfrak{h}\right)\right]^{\perp}, i=1,2
$$

where the ortogonal complement is taken in $\mathfrak{h}$ with respect to the form $(.,$.$) . One proves$ [1, 7] that $\mathfrak{m}_{ \pm}$are the orthogonal complements to $\mathfrak{g}_{ \pm}$which implies

$$
\mathfrak{h}_{i}^{\text {ort }} \subset \mathfrak{h}_{i}, i=1,2 \text {. }
$$


Therefore $\mathfrak{g}_{ \pm} / \mathfrak{m}_{ \pm}$can be identified with $\mathfrak{l}_{1,2}^{\prime} \oplus\left(\mathfrak{h}_{+} / \mathfrak{h}_{+}^{\text {ort }}\right)$ and the Cayley transform $\theta$ can be considered as an orthogonal Lie algebra isomorphism between the last two algebras. One can choose complements $\mathfrak{a}_{i}$ for $\mathfrak{h}_{i}^{\text {ort }}$ in $\mathfrak{h}_{i}(i=1,2)$, for which the map $\theta$ can be lifted to a Lie algebra isomorphism

$$
\theta: \mathfrak{g}_{1}^{\prime} \oplus \mathfrak{a}_{1} \rightarrow \mathfrak{g}_{2}^{\prime} \oplus \mathfrak{a}_{2}
$$

preserving the form $(.,$.$) in such a way that \theta\left(\mathfrak{a}_{1}\right)=\mathfrak{a}_{2}$.

Proposition 3.3 For a Belavin-Drinfeld triple $\left(\Gamma_{1}, \Gamma_{2}, \tau\right)$ the algebras $\mathfrak{g}_{ \pm}, \mathfrak{m}_{ \pm}$, are given by

$$
\begin{aligned}
& \mathfrak{m}_{ \pm}=\mathfrak{h}_{1,2}^{\text {ort }} \dot{+} \mathfrak{n}_{ \pm}, \\
& \mathfrak{g}_{ \pm}=\left(\mathfrak{l}_{1,2}^{\prime} \oplus \mathfrak{a}_{1,2}\right) \dot{+} \mathfrak{m}_{ \pm}
\end{aligned}
$$

and thus the quotients $\mathfrak{g}_{ \pm} / \mathfrak{m}_{ \pm}$are

$$
\mathfrak{g}_{ \pm} / \mathfrak{m}_{ \pm} \cong \mathfrak{l}_{1,2}^{\prime} \oplus \mathfrak{a}_{1,2}
$$

The Cayley transform $\theta: \mathfrak{l}_{1}^{\prime} \oplus \mathfrak{a}_{1} \rightarrow \mathfrak{l}_{2}^{\prime} \oplus \mathfrak{a}_{2}$ maps $\mathfrak{l}_{1}^{\prime}$ to $\mathfrak{l}_{2}^{\prime}$ extending the map $\tau$ to root spaces and $\mathfrak{a}_{1}$ to $\mathfrak{a}_{2}$ preserving the form (.,.) (where it is completely determined by the term $r_{0}$ of the r-matrix $r$ ).

The projections $\mathrm{p}_{ \pm}: \mathfrak{g}_{ \pm} \rightarrow \mathfrak{l}_{1,2}^{\prime} \oplus \mathfrak{a}_{1,2} \cong \mathfrak{g}_{ \pm} / \mathfrak{m}_{ \pm}$(recall eq. (3.7)) are just the projections on the first components in (3.8).

The decompositions (3.8) are similar to the Levi decompositions of $\mathfrak{g}_{ \pm}$. The Lie algebras $\mathfrak{m}_{ \pm}$stay "between" the nilpotent radicals $\mathfrak{n}_{ \pm}$of $\mathfrak{g}_{ \pm}$and their radicals $\mathfrak{z} 1,2+\mathfrak{n}_{ \pm}$.

Example 3.4 Consider the special case when the $r$-matrix $r$ is such that

$$
\mathfrak{g}_{+} \supset \mathfrak{h}
$$

This means that the Cartan subalgebra $\mathfrak{h}$ sits entirely inside the subalgebra of $\mathfrak{g}$ spanned by the first components of $r$ and is equivalent to the condition $\mathfrak{h}_{i}^{\text {ort }}=0$. Then $\mathfrak{g}_{ \pm}$are full parabolic subalgebras

$$
\mathfrak{g}_{ \pm}=\mathfrak{p}_{ \pm}
$$

and their ideals $\mathfrak{m}_{ \pm}$are equal to the unipotent radicals $\mathfrak{n}_{ \pm}$of $\mathfrak{p}_{ \pm}$

$$
\mathfrak{m}_{ \pm}=\mathfrak{n}_{ \pm}
$$

The quotient algebras $\mathfrak{g}_{ \pm} / \mathfrak{m}_{ \pm}$are isomorphic to the Levi factors $\mathfrak{l}_{1,2}$ of $\mathfrak{p}_{ \pm}:$

$$
\mathfrak{g}_{ \pm} / \mathfrak{m}_{ \pm} \cong \mathfrak{l}_{1,2}
$$

So $\mathfrak{a}_{i}$ can be naturally taken $\mathfrak{z}_{i}(i=1,2)$ and the Cayley transform $\theta: \mathfrak{l}_{1} \rightarrow \mathfrak{l}_{2}$ is an orthogonal Lie algebra isomorphism between the full Levi factors $\mathfrak{l}_{1}$ and $\mathfrak{l}_{2}$. 
Example 3.5 The standard Lie bialgebra structure on a complex semisimple Lie algebra $\mathfrak{g}$ comes from the $r$-matrix

$$
r=\sum_{x_{1}} x_{i} \otimes x_{i}+\sum_{\alpha \in \Delta_{+}} x_{-\alpha} \otimes x_{\alpha}
$$

where $\left\{x_{i}\right\}$ is an orthonormal basis of $\mathfrak{h}$ with respect to the Killing form on $\mathfrak{g}$. It is associated with the trivial Belavin-Drinfeld triple for which $\Gamma_{1}=\Gamma_{2}=\emptyset$. The algebras $\mathfrak{g}_{ \pm}$are equal to the Borel subalgebras $\mathfrak{b}_{ \pm}$, and $\mathfrak{m}_{ \pm}$are their unipotent radicals $\mathfrak{n}_{ \pm}$. The quotients $\mathfrak{g}_{ \pm} / \mathfrak{m}_{ \pm}$are naturally isomorphic to the Cartan subalgebra $\mathfrak{h}\left(\mathfrak{l}_{i}^{\prime}\right.$ and $\mathfrak{h}_{i}^{\text {ort }}, i=1,2$ are trivial). Under this identification the map $\theta: \mathfrak{h} \rightarrow \mathfrak{h}$ is $\theta=-\mathrm{id}_{\mathfrak{h}}$.

Example 3.6 Let $\mathfrak{g}=\mathfrak{s l}(n+1), \mathfrak{h}$ be its Cartan subalgebra consisting of diagonal matrices, and $\left\{\alpha_{1}, \ldots, \alpha_{n}\right\}$ be the set of its simple roots.

The Cremmer-Gervais [3, 9] Lie bialgebra structure on it is associated to the BelavinDrinfeld triple $\Gamma_{1}=\left\{\alpha_{1}, \ldots, \alpha_{n-1}\right\}, \Gamma_{2}=\left\{\alpha_{2}, \ldots, \alpha_{n}\right\}, \tau\left(\alpha_{j}\right)=\alpha_{j+1}, j=1, \ldots, n-1$. The algebras $\mathfrak{h}_{i}^{\text {ort }}$ are trivial and

$$
\begin{aligned}
& \mathfrak{l}_{1}=\{\operatorname{diag}(A,-\operatorname{tr} A) \mid A \in \mathfrak{g l}(n)\} \cong \mathfrak{g l}(n), \\
& \mathfrak{l}_{2}=\{\operatorname{diag}(-\operatorname{tr} A, A) \mid A \in \mathfrak{g l}(n)\} \cong \mathfrak{g l}(n)
\end{aligned}
$$

(in block notation). The $r_{0}$ component of the $r$-matrix is chosen in such a way that its Cayley transform $\theta: \mathfrak{l}_{1} \rightarrow \mathfrak{l}_{2}$ is

$$
\theta(\operatorname{diag}(A,-\operatorname{tr} A))=\operatorname{diag}(-\operatorname{tr} A, A) .
$$

\subsection{Related Poisson-Lie groups}

For a complex reductive group $R$ we will denote its derived group by $R^{\prime}$ and the identity component of its center by $Z(R)^{\circ}$. Recall the standard fact

$$
R=R^{\prime} Z(R)^{\circ}
$$

and that $R^{\prime} \cap Z(R)^{\circ}$ is a finite abelian group.

In the rest of this paper $G$ will denote a connected complex reductive Lie group. We will fix a factorizable Lie bialgebra structure on $\mathfrak{g}:=\operatorname{Lie}(G)$ associated to a Belavin-Drinfeld triple $\left(\Gamma_{1}, \Gamma_{2}, \tau\right)$ and equip $G$ with the corresponding Sklyanin Poisson-Lie structure (see (1.8)).

With $G_{+}$and $G_{-}$we will denote the connected subgroups of $G$ with tangent Lie bialgebras $\mathfrak{g}_{+}$and $\mathfrak{g}_{-}$. They are Poisson-Lie subgroups of $G$ since $\mathfrak{g}_{ \pm}$are Lie sub-bialgebras of $\mathfrak{g}$. Let $M_{ \pm}$be the subgroups of $G_{+}$with $\operatorname{Lie}\left(M_{ \pm}\right)=\mathfrak{m}_{ \pm}$.

Assuming the notation from Subsect. 3.1 for $\operatorname{Lie}(G)=\mathfrak{g}$, we will give explicit formulas for $M_{ \pm}$and $G_{ \pm}$, similar to (3.7) and (3.8). The maximal torus of $G$ corresponding to the fixed Cartan subalgebra $\mathfrak{h}$ of $\mathfrak{g}$ will be denoted by $H$. Let $B_{ \pm}$be the Borel subgroups of $G$ relative to $H$ with $\operatorname{Lie}\left(B_{ \pm}\right)=\mathfrak{b}_{ \pm}$. Denote with $P_{ \pm}$the parabolic subgroups of $G$ with Lie algebras $\mathfrak{p}_{ \pm}$. Let $L_{1,2}$ be their Levi factors and $N_{ \pm}$be their unipotent radicals. The Levi decompositions of $P_{ \pm}$are

$$
P_{ \pm}=L_{1,2} \ltimes N_{ \pm} .
$$


The connected subgroups of $Z\left(L_{i}\right)^{\circ}$ with Lie algebras $\mathfrak{a}_{i}$ and $\mathfrak{h}_{i}^{\text {ort }}$ will be denoted by $A_{i}$ and $H_{i}^{\text {ort }}$.

The group analogs of formulas (3.7) and (3.8) are

$$
\begin{aligned}
& M_{ \pm}=H_{1,2}^{\text {ort }} \ltimes N_{ \pm}, \\
& G_{ \pm}=\left(L_{1,2}^{\prime} A_{1,2}\right) M_{ \pm} .
\end{aligned}
$$

The intersections $L_{i}^{\prime} \cap A_{i} \subset Z\left(L_{i}^{\prime}\right)$ are finite groups, but they are nontrivial in general. The groups $L_{1,2}^{\prime} A_{1,2}$ normalize $M_{ \pm}$. The intersections

$$
\left(L_{1,2}^{\prime} A_{1,2}\right) \cap M_{ \pm}=\left(Z\left(L_{1,2}^{\prime}\right) A_{1,2}\right) \cap H_{1,2}^{\text {ort }}
$$

are discrete subgroups of $Z\left(L_{1,2}^{\prime} A_{1,2}\right)$. Generally they are also nontrivial.

The factor groups $G_{ \pm} / M_{ \pm}$are

$$
\left.G_{ \pm} / M_{ \pm} \cong\left(L_{1,2}^{\prime} A_{1,2}\right) /\left(Z\left(L_{1,2}^{\prime}\right) A_{1,2} \cap H_{1,2}^{\text {ort }}\right)\right) .
$$

Unfortunately the Cayley transform $\theta: \mathfrak{l}_{1}^{\prime} \oplus \mathfrak{a}_{1} \rightarrow \mathfrak{l}_{2}^{\prime} \oplus \mathfrak{a}_{2}$ cannot be lifted in general to an isomorphism between the two groups in (3.15). One of the reasons for this is that it migth not map the kernel of the exponential map for $L_{1}^{\prime} A_{1}$ to the one of the exponential map for $L_{2}^{\prime} A_{2}$. Nevertheless there exist two discrete subgroups $\Lambda_{i}$ of $Z\left(L_{i}^{\prime} A_{i}\right)$ containing $\left(L_{i}^{\prime} A_{i}\right) \cap H_{i}^{\text {ort }}$ such that the factor groups $\left(L_{i}^{\prime} A_{i}\right) / \Lambda_{i}$ are isomorphic (as abstract groups only). We will consider the minimal such groups $\Lambda_{i}$. Explicitly they are given by

$$
\Lambda_{i}=\left(\exp _{i} \circ \theta^{-\epsilon_{i}} \circ \exp _{3-i}^{-1}\left(Z\left(L_{3-i}^{\prime}\right) A_{3-i} \cap H_{3-i}^{\text {ort }}\right)\right)\left(Z\left(L_{i}^{\prime}\right) A_{i} \cap H_{i}^{\text {ort }}\right)
$$

where $\epsilon_{i}=(-1)^{i}, i=1,2$. The corresponding isomorphism will be denoted by $\Theta$ :

$$
\Theta:\left(L_{1}^{\prime} A_{1}\right) / \Lambda_{1} \rightarrow\left(L_{2}^{\prime} A_{2}\right) / \Lambda_{2} .
$$

Clearly the groups $\Lambda_{i}$ consist of semisimple elements of $L_{i}^{\prime} A_{i}$. Thus the restrictions of the projections $L_{i}^{\prime} A_{i} \rightarrow\left(L_{i}^{\prime} A_{i}\right) / \Lambda_{i}$ to any unipotent subgroup of $L_{i}^{\prime} A_{i}$ are one to one.

Remark 3.7 Note that the groups $\Lambda_{i}$ might have in general some accumulation points and then $\left(L_{i}^{\prime} A_{i}\right) / \Lambda_{i}$ will not have structure of differential manifolds. In Sect. 3.2.1 and Sect. 4 we will assume that the $r$-matrix, we start with, is such that this does not happen. In Sect. 5 the full generality will be assumed. It brings the inconvenience that $G^{r}$ is not a closed subgroup of $G \times G$, but this will not be essential for the proofs there.

Let

$$
\mathrm{p}_{ \pm}: G_{ \pm} \rightarrow\left(L_{1,2} A_{1,2}\right) / \Lambda_{1,2}
$$

denote the compositions of the projection maps

$$
\left.G_{ \pm} \rightarrow G_{ \pm} / M_{ \pm} \cong\left(L_{1,2}^{\prime} A_{1,2}\right) /\left(\left(L_{1,2}^{\prime} A_{1,2}\right) \cap H_{1,2}^{\text {ort }}\right)\right) \rightarrow\left(L_{1,2}^{\prime} A_{1,2}\right) / \Lambda_{1,2}
$$

Their differentials at the origin are equal to the maps $\mathrm{p}_{ \pm}: \mathfrak{g}_{ \pm} \rightarrow \mathfrak{g}_{ \pm} / \mathfrak{m}_{ \pm}$introduced in Sect. 2. 
Remark 3.8 If the Poisson structure on $G$ comes from an $r$-matrix $r$ satisfying $\mathfrak{g}_{+} \supset \mathfrak{h}$ (see Example 3.4), then the groups $G_{ \pm}$are the full parabolic subgroups $P_{ \pm}$of $G, M_{ \pm}$are their unipotent radicals $N_{ \pm}$, and $L_{1,2}^{\prime} A_{1,2}$ are their Levi factors $L_{1,2}$. ( $H_{1,2}^{\text {ort }}$ are trivial.) The groups $\Lambda_{1,2}$ are generally nontrivial even if $P_{ \pm}=B_{ \pm}$and $L_{i}=H$.

Example 3.9 The standard Poisson structure on a semisimple Lie group $G$ is the integration of the standard Lie bialgebra structure on $\mathfrak{g}=\operatorname{Lie}(G)$ reviewed in Example 3.5. In this case $G_{ \pm}=B_{ \pm}, M_{ \pm}=N_{ \pm}$where $N_{ \pm}$are the unipotent radicals of the Borel subgroups $B_{ \pm}$. The groups $L_{1,2}^{\prime}, H_{1,2}^{\text {ort }}$ are trivial and $A_{1,2}=H$. There is no need to introduce additional groups $\Lambda_{i}$ and the Cayley transform $\Theta: H \rightarrow H$ acts as $\Theta(h)=h^{-1}$.

Example 3.10 The groups $L_{i}$ for the Cremmer-Gervais Poisson structure on $S L(n+1)$ (see Example 3.6) are

$$
\begin{aligned}
& L_{1}=\left\{\operatorname{diag}\left(A,(\operatorname{det} A)^{-1}\right) \mid A \in G L(n)\right\} \cong G L(n), \\
& L_{2}=\left\{\operatorname{diag}\left((\operatorname{det} A)^{-1}, A\right) \mid A \in G L(n)\right\} \cong G L(n) .
\end{aligned}
$$

$G_{ \pm}$are the parabolic subgroups of $S L(n+1)$ containing $B_{ \pm}$with Levi factors $L_{1,2}$ respectively. The map $\theta: \mathfrak{l}_{1} \rightarrow \mathfrak{l}_{2}$ lifts to $\Theta: L_{1} \rightarrow L_{2}:$

$$
\Theta\left(\operatorname{diag}\left(A,(\operatorname{det} A)^{-1}\right)\right)=\operatorname{diag}\left((\operatorname{det} A)^{-1}, A\right),
$$

so $\Lambda_{i}$ are trivial.

\subsubsection{The double $\mathrm{D}\left(G_{+}\right)$}

Combining the results from Subsect. 2.2 and eq. (3.15), we see that we can choose as a double Poisson-Lie group $\mathrm{D}\left(G_{+}\right)$of $G_{+}$the following group

$$
\mathrm{D}\left(G_{+}\right):=G \times\left(\left(L_{1}^{\prime} A_{1}\right) / \Lambda_{1}\right) .
$$

The groups $G_{+}$and $G_{-}$are embedded in it by

$$
\begin{aligned}
& i_{+}(x)=\left(x, \mathrm{p}_{+}(x)\right), x \in G_{+}, \\
& i_{-}(y)=\left(y, \Theta^{-1} \circ \mathrm{p}_{-}(x)\right), y \in G_{-}
\end{aligned}
$$

(cf. Proposition 2.3). The group (3.17) is also a double of $G_{-}$, but the corresponding Poisson bivector field is opposite to the one for $\mathrm{D}\left(G_{+}\right)$since $\mathfrak{g}_{-} \cong \mathfrak{g}_{+}^{* \text { op }}$.

\subsubsection{The double $\mathrm{D}(G)$}

The results from Subsect. 2.1 imply that the double Poisson-Lie group $\mathrm{D}(G)$ of $G$ can be chosen as

$$
\mathrm{D}(G):=G \times G .
$$

$G$ is embedded in it diagonally:

$$
d: G \rightarrow G \times G, \quad d(g)=(g, g), \text { for } g \in G .
$$


Its image will be denoted by $d(G)$. The connected subgroup of $G \times G$ with Lie algebra $\mathfrak{g}^{r}$ will be denoted by $G^{r}$ (see Propositions 2.1 and 2.2). It plays the role of opposite dual Poisson-Lie group of $G$ (see Theorem 1.9) and is explicitly given by

$$
G^{r}:=\left\{\left(g_{+}, g_{-}\right) \mid g_{+} \in G_{+}, g_{-} \in G_{-} \text {, and } \Theta \circ \mathrm{p}_{1}\left(g_{+}\right)=\mathrm{p}_{-}\left(g_{-}\right)\right\} .
$$

Because of Proposition 2.2 we need only to prove that the group in (3.20) is connected. Consider its identity component $\left(G^{r}\right)^{\circ}$ and define $\check{\Lambda}_{i} \subset L_{i}^{\prime} A_{i}$ by

$$
\check{\Lambda}_{i}=\left\{x \in L_{i}^{\prime} A_{i} \mid(x, e) \in\left(G^{r}\right)^{\circ}\right\}, i=1,2 .
$$

The following map is an isomorphism from $\left(L_{1}^{\prime} A_{1}\right) / \check{\Lambda}_{1}$ to $\left(L_{2}^{\prime} A_{2}\right) / \check{\Lambda}_{2}$ :

$$
l_{1} \check{\Lambda}_{1} \mapsto l_{2} \check{\Lambda}_{2} \text {, if } l_{i} \in L_{i}^{\prime} A_{i} \text {, and }\left(l_{1}, l_{2}\right) \in\left(G^{r}\right)^{\circ} .
$$

It is correctly defined since $L_{1}^{\prime} A_{1}$ is connected. Besides this $\check{\Lambda}_{i} \supset\left(L_{i}^{\prime} A_{i}\right) \cap H_{i}^{\text {ort }}$ because $H_{1}^{\text {ort }} \times e$ and $e \times H_{2}^{\text {ort }}$ are subgroups of $\left(G^{r}\right)^{\circ}$. From the minimality property of $\Lambda_{i}$ we get $\Lambda_{i} \equiv \check{\Lambda}_{i}$ which implies $G^{r}=\left(G^{r}\right)^{\circ}$.

\subsection{Induction on factorizable complex reductive Lie bialgebras and Poisson-Lie groups}

As in Subsect. 3.1 we will assume that $\mathfrak{g}$ is a complex reductive Lie bialgebra with an $r$ matrix as in (3.2) associated to the Belavin-Drinfeld triple $\left(\Gamma_{1}, \Gamma_{2}, \tau\right)$. Recall that the nilpotency condition for the map $\tau$ states that for each $\gamma \in \Gamma_{1}$ there exists an integer $n(\gamma)$, such that $\tau^{n(\gamma)} \in \Gamma_{2} \backslash \Gamma_{1}$. Denote

$$
\operatorname{ord}(\tau)=\max _{\gamma \in \Gamma_{1}} n(\gamma)
$$

We will canonically construct a chain of complex reductive factorizable Lie bialgebras $\mathfrak{g}^{(k)}(k=0, \ldots, \operatorname{ord}(\tau))$ associated to Belavin-Drinfeld triples $\left(\Gamma_{1}^{(k)}, \Gamma_{2}^{(k)}, \tau^{(k)}\right)$ such that $\mathfrak{g}^{(0)} \equiv \mathfrak{g}$ as Lie bialgebras and

$$
\operatorname{ord}\left(\tau^{(k)}\right)=\operatorname{ord}(\tau)-k
$$

Recall that $\mathrm{D}\left(\mathfrak{g}_{+}\right) \cong \mathfrak{g} \oplus\left(\mathfrak{l}_{1}^{\prime} \oplus \mathfrak{a}_{1}\right)$ and $\mathfrak{g}_{+}$and $\mathfrak{g}_{-}$are embedded in it via the maps $i_{ \pm}$ (2.9)-2.10). Let $\mathfrak{d}$ be the subalgebra of $\mathfrak{g} \oplus\left(\mathfrak{l}_{1}^{\prime} \oplus \mathfrak{a}_{1}\right)$ defined by

$$
\mathfrak{d}=\mathfrak{l}_{2} \oplus\left(\left(\mathfrak{l}_{1}^{\prime} \oplus \mathfrak{a}_{1}\right) \cap \mathfrak{l}_{2}\right)=\mathfrak{l}_{2} \oplus\left(\left(\mathfrak{l}_{1}^{\prime} \cap \mathfrak{l}_{2}\right) \oplus \mathfrak{a}_{1}\right) .
$$

It is clear that the restriction of the standard bilinear form of $\mathrm{D}\left(\mathfrak{g}_{+}\right)$(see the proof of Proposition 2.3) to $\mathfrak{d}$ is nondegenerate. Define

$$
\begin{aligned}
& \mathfrak{g}_{+}^{(1)}=\left(\left(\mathfrak{l}_{1}^{\prime} \cap \mathfrak{l}_{2}\right) \oplus \mathfrak{a}_{1}\right) \dot{+}\left(\mathfrak{h}_{1}^{\text {ort }} \dot{+} \mathfrak{n}_{+}^{(1)}\right) \subset \mathfrak{g}_{+}, \\
& \mathfrak{g}_{-}^{(1)}=\left(\theta\left(\mathfrak{l}_{1}^{\prime} \cap \mathfrak{l}_{2}\right) \oplus \mathfrak{a}_{2}\right) \dot{+}\left(\mathfrak{h}_{2}^{\text {ort }} \dot{+} \mathfrak{n}_{-}^{(1)}\right) \subset \mathfrak{g}_{-}
\end{aligned}
$$

where $\mathfrak{n}_{+}^{(1)}, \mathfrak{n}_{-}^{(1)}$ are the nilpotent subalgebras of $\mathfrak{l}_{2}^{\prime}$ spanned by the root spaces of positive / negative roots of $\mathfrak{l}_{2}^{\prime}$ that are not roots of $\mathfrak{l}_{1}^{\prime} \cap \mathfrak{l}_{2}^{\prime}, \theta\left(\mathfrak{l}_{1}^{\prime} \cap \mathfrak{l}_{2}^{\prime}\right)$. 
The images $i_{ \pm}\left(\mathfrak{g}_{ \pm}^{(1)}\right)$ of $\mathfrak{g}_{ \pm}^{(1)}$ under the inclusions $i_{ \pm}$(see (2.9)-(2.10)) are isotropic subalgebras of $\mathfrak{d}$ with trivial intersection since the same is true for the full images $i_{ \pm}\left(\mathfrak{g}_{ \pm}\right)$as subalgebras of $\mathrm{D}\left(\mathfrak{g}_{+}\right)$. Taking into account $\operatorname{dim} \mathfrak{d}=2 \operatorname{dim} \mathfrak{g}_{ \pm}^{(1)}$ we get that $\left(\mathfrak{d}, i_{+}\left(\mathfrak{g}_{+}^{(1)}\right), i_{-}\left(\mathfrak{g}_{-}^{(1)}\right)\right)$ is a Manin triple. This induces Lie bialgebra structures on $\mathfrak{g}_{ \pm}^{(1)}$ and $\mathfrak{d}$ such that $\mathfrak{d} \cong \mathrm{D}\left(\mathfrak{g}_{ \pm}^{(1)}\right)$. One easily checks that the second term of $\mathfrak{d}$ in (3.21) is a Lie bialgebra ideal of $\mathfrak{d}$. The factor of $\mathfrak{d}$ by it is isomorphic to $\mathfrak{l}_{2}$ as a Lie algebra which induces a Lie bialgebra structure on $\mathfrak{l}_{2}$. Set

$$
\mathfrak{g}^{(1)}:=\mathfrak{l}_{2} .
$$

Define the subsets $\Gamma_{1}^{(1)}$ and $\Gamma_{2}^{(1)}$ of $\Gamma_{2}$ by

$$
\begin{aligned}
& \Gamma_{1}^{(1)}=\Gamma_{1} \cap \Gamma_{2}, \\
& \Gamma_{2}^{(1)}=\tau\left(\Gamma_{1} \cap \Gamma_{2}\right),
\end{aligned}
$$

and let

$$
\tau^{(1)}:=\left.\tau\right|_{\Gamma_{1}^{(1)}}: \Gamma_{1}^{(1)} \rightarrow \Gamma_{2}^{(1)}
$$

The definition of the Lie bialgebra structure on $\mathfrak{g}^{(1)}$ as a quotient of the double $\mathfrak{d}$ (3.21) implies that it comes from the $r$-matrix

$$
r^{(1)}=r_{0}+\sum_{\alpha \in \Delta_{2+}} x_{-\alpha} \otimes x_{\alpha}+\sum_{\alpha, \beta \in \Delta_{2+}, \alpha<\beta} x_{-\alpha} \wedge x_{\beta}
$$

where $\Delta_{2+}$ denotes the set of positive roots of $\mathfrak{l}_{2}$ and $<$ is the restriction of the initial partial ordering of $\Delta_{+}$to $\Delta_{2+}$ (see Theorem 3.2). The Belavin-Drinfeld triple associated to the $r$-matrix $r^{(1)}$ is $\left(\Gamma_{1}^{(1)}, \Gamma_{1}^{(1)}, \tau^{(1)}\right)$. The images of the corresponding homomorphisms $r_{ \pm}^{(1)}:\left(\mathfrak{g}^{(1)}\right)^{*} \rightarrow \mathfrak{g}^{(1)}$ are exactly the Lie bialgebras $\mathfrak{g}_{+}^{(1)}$ and $\mathfrak{g}_{-}^{(1)}$. From (3.24) it is clear that

$$
\operatorname{ord}\left(\tau^{(1)}\right)=\operatorname{ord}(\tau)-1 \text {. }
$$

Repeatedly applying this construction, one builds a sequence $\mathfrak{g}^{(0)}=\mathfrak{g}, \mathfrak{g}^{(1)}, \ldots, \mathfrak{g}^{(\text {ord } \tau)}$ of reductive factorizable Lie bialgebras with the stated property.

The Lie bialgebra $\mathfrak{g}^{(\text {ord } \tau)}$ obtained at the end is the Cartan subalgebra $\mathfrak{h}$ of $\mathfrak{g}$ with trivial Lie cobracket (since it is an abelian coboundary bialgebra). Besides this $\mathfrak{g}_{ \pm}^{(\operatorname{ord} \tau)}$ and $\mathrm{D}\left(\mathfrak{g}^{(\operatorname{ord} \tau)}\right)$ are given by

$$
\begin{aligned}
& \mathfrak{g}_{ \pm}^{(\operatorname{ord} \tau)}=\mathfrak{g}_{ \pm} \cap \mathfrak{h}, \\
& \mathrm{D}\left(\mathfrak{g}^{(\operatorname{ord} \tau)}\right)=\mathfrak{h} \oplus\left(\left(\mathfrak{l}_{1}^{\prime} \oplus \mathfrak{a}_{1}\right) \cap \mathfrak{h}\right)=\mathfrak{h} \oplus\left(\left(\mathfrak{l}_{1}^{\prime} \cap \mathfrak{h}\right) \oplus \mathfrak{a}_{1}\right) .
\end{aligned}
$$

On the group level, one constructs a chain of connected complex reductive Poisson-Lie groups $G^{(0)}=G, G^{(1)}, \ldots, G^{(\operatorname{ord} \tau)}=H$. As a Lie group $G^{(1)}$ is just $L_{2}$ and $G_{ \pm}^{(1)}$ are given by

$$
G_{ \pm}^{(1)}=\left(L_{1,2}^{(1)} A_{1,2}^{(1)}\right)\left(H_{1,2}^{\text {ort }} \ltimes N_{ \pm}^{(1)}\right),
$$

where $L_{1}^{(1)}, L_{2}^{(1)}, A_{1}^{(1)}$, and $A_{2}^{(1)}$ denote the connected subgroups of $G$ with Lie algebras $\mathfrak{l}_{1}^{\prime} \cap \mathfrak{l}_{2}^{\prime}, \theta\left(\mathfrak{l}_{1}^{\prime} \cap \mathfrak{l}_{2}^{\prime}\right),\left(\mathfrak{l}_{1}^{\prime} \cap \mathfrak{z}_{2}\right) \oplus \mathfrak{a}_{1}$, and $\theta\left(\mathfrak{l}_{1}^{\prime} \cap \mathfrak{z}_{2}\right) \oplus \mathfrak{a}_{2} . N_{ \pm}^{(1)}$ are the unipotent subgroups of $G$ with Lie algebras $\mathfrak{n}_{ \pm}^{(1)}$. 
The corresponding groups $M_{ \pm}^{(1)} \subset G_{ \pm}^{(1)}$ (see Subsect. 3.2) are the second factors in these formulas.

On Lie algebra level the Cayley transform $\theta^{(1)}: \operatorname{Lie}\left(L_{1}^{(1)} A_{1}^{(1)}\right) \rightarrow \operatorname{Lie}\left(L_{2}^{(1)} A_{2}^{(1)}\right)$ is the restriction of $\theta$ to the subspace $\operatorname{Lie}\left(L_{1}^{(1)} A_{1}^{(1)}\right)=\left(\mathfrak{l}_{1}^{\prime} \oplus \mathfrak{a}_{1}\right) \cap \mathfrak{l}_{2}$ of $\mathfrak{l}_{1}^{\prime} \oplus \mathfrak{a}_{1}$. Since $\Lambda_{i}$ are subgroups of $Z\left(L_{i}^{\prime} A_{i}\right) \subset H$ they sit inside $L_{i}^{(1)} A_{i}^{(1)}$ and $\theta^{(1)}$ can be lifted to an isomorphism

$$
\Theta^{(1)}:\left(L_{1}^{(1)} A_{1}^{(1)}\right) / \Lambda_{1} \rightarrow\left(L_{2}^{(1)} A_{2}^{(1)}\right) / \Lambda_{2} .
$$

The double $\mathrm{D}\left(G_{ \pm}^{1}\right)$ can be taken as

$$
\mathrm{D}\left(G_{ \pm}^{(1)}\right):=G^{(1)} \times\left(L_{1}^{(1)} A_{1}^{(1)} / \Lambda_{1}\right) .
$$

It is naturally a subgroup of $G \times\left(L_{1} A_{1} / \Lambda_{1}\right)$ and the embeddings

$$
i_{ \pm}^{(1)}: G_{ \pm}^{(1)} \rightarrow \mathrm{D}\left(G^{(1)}\right)
$$

are simply the restrictions of the embeddings $i_{ \pm}$(see $\left.(3.18)-(3.19)\right)$ to $G_{ \pm}^{(1)}$.

The last object $G^{(\operatorname{ord} \tau)}$ is

$$
G^{(\operatorname{ord} \tau)}=H
$$

and

$$
G_{ \pm}^{(\operatorname{ord} \tau)}=\left(L_{1,2}^{\prime} \cap H\right) A_{1,2} H_{1,2}^{\text {ort }} .
$$

At each step of the described procedure there is a second canonical way of constructing a Lie bialgebra $\mathfrak{g}^{k+1}$ from $\mathfrak{g}^{k}$, having the stated property. Restricting to the first step $(k=0)$, define $\mathfrak{g}^{(1)}=\mathfrak{l}_{1}$,

$$
\begin{aligned}
& \mathfrak{g}_{+}^{(1)}=\left(\theta^{-1}\left(\mathfrak{l}_{2}^{\prime} \cap \mathfrak{l}_{1}\right) \oplus \mathfrak{a}_{1}\right) \dot{+}\left(\mathfrak{h}_{1}^{\text {ort }} \dot{+} \theta^{-1}\left(\mathfrak{n}_{1}^{(1)}\right)\right), \\
& \mathfrak{g}_{-}^{(1)}=\left(\mathfrak{l}_{2}^{\prime} \cap \mathfrak{l}_{1} \oplus \mathfrak{a}_{2}\right) \dot{+}\left(\mathfrak{h}_{2}^{\text {ort }} \dot{+} \theta^{-1}\left(\mathfrak{n}_{1}^{(1)}\right)\right),
\end{aligned}
$$

and induce Lie bialgebra structure on $\mathfrak{g}^{(1)}$ using the subalgebra $\mathfrak{l}_{1} \oplus\left(\left(\mathfrak{l}_{2}^{\prime} \oplus \mathfrak{a}_{2}\right) \cap \mathfrak{l}_{1}\right)$ of $\mathrm{D}\left(\mathfrak{g}_{+}\right)$. This structure comes from an $r$-matrix as in (3.25) with sums over the roots of $\mathfrak{l}_{1}^{\prime}$ insted of $\mathfrak{l}_{2}^{\prime}$. The two choices for Lie bialgebras $\mathfrak{g}^{(1)}$ are isomorphic if $\mathfrak{h}_{ \pm}^{\text {ort }}$ are trivial, but seem to be slightly different in general.

\section{Symplectic leaves in $G_{-}$}

Subsect. 4.1 is devoted to the description of the set of $i_{+}\left(G_{+}\right)$double cosets of $\mathrm{D}\left(G_{+}\right)$, while Subsect. 4.2 studies the discrete subgroup $i_{-}\left(G_{-}\right) \cap i_{+}\left(G_{+}\right)$of $\mathrm{D}\left(G_{+}\right)$. In the last Subsection these results are combined with Theorem 1.10 to describe the set of symplectic leaves of $G_{-}$.

\subsection{Double cosets of $i_{+}\left(G_{+}\right)$in $\mathrm{D}\left(G_{+}\right)$}

Denote the double coset of the element

$$
(g, \tilde{l}) \in G \times\left(\left(L_{1}^{\prime} A_{1}\right) / \Lambda_{1}\right) \cong \mathrm{D}\left(G_{+}\right)
$$


by $[(g, \tilde{l})]$ :

$$
[(g, \tilde{l})]=i_{+}\left(G_{+}\right)(g, \tilde{l}) i_{+}\left(G_{+}\right) .
$$

Since $L_{1}^{\prime} A_{1} \subset G_{+}$, for all $g \in G, \tilde{l} \in L_{1}^{\prime} A_{1}$

$$
\left[\left(g, \mathrm{p}_{+}(l) \tilde{l}\right)\right]=\left[\left(l^{-1} g, \tilde{l}\right)\right] \text { and }\left[\left(g, \tilde{l} \mathrm{p}_{+}(l)\right)\right]=\left[\left(g l^{-1}, \tilde{l}\right)\right], \forall l \in L_{1}^{\prime} A_{1} .
$$

The following Proposition illustrates the problem of classifying double cosets.

Proposition 4.1 The $i_{+}\left(G_{+}\right)$double cosets of $\mathrm{D}\left(G_{+}\right)$are in one to one correspondence with the conjugation orbits of $G_{+}$in $G /\left(\Lambda_{1} M_{+}\right)$.

Recall that $M_{+}$is a normal subgroup of $G_{+}$and $\Lambda_{1} \in Z\left(L_{1}\right)$, so the conjugation action of $G_{+}$on $G$ indeed can be pushed down to the the homogeneous space $G / \Lambda_{1} M_{+}$. Note also that this action can equivalently be described as the action of $\left(L_{1}^{\prime} A_{1}\right) M_{+}$on $G /\left(\Lambda_{1} M_{+}\right)$ where the first factor acts by conjugation and the second one by left multiplication.

Proof of Proposition 4.1. By a straightforward computation for any $g \in G$

$$
\begin{aligned}
{[(g, e)] } & =\left\{\left(m^{\prime} l^{\prime} g l^{\prime \prime} m^{\prime \prime}, \mathrm{p}_{+}\left(l^{\prime}\right) \mathrm{p}_{+}\left(l^{\prime \prime}\right)\right) \mid l^{\prime}, l^{\prime \prime} \in L_{1}^{\prime} A_{1}, m^{\prime}, m^{\prime \prime} \in M_{+}\right\} \\
& =\left\{\left(m^{\prime} l^{\prime} g\left(l^{\prime}\right)^{-1} m^{\prime \prime} k, \mathrm{p}_{+}(k)\right) \mid l^{\prime}, k \in L_{1}^{\prime} A_{1}, m^{\prime}, m^{\prime \prime} \in M_{+}\right\} .
\end{aligned}
$$

The second expression is obtained from the first by letting $l^{\prime} l^{\prime \prime}=k \in L_{1}^{\prime} A_{1}$ and expressing $l^{\prime \prime}$ in terms of $l^{\prime}$ and $k$. We also used the fact that $M_{+}$is a normal subgroup of $G_{+}$.

To the $G_{+}$conjugation orbit of $g\left(\Lambda_{1} M_{+}\right)$we associate the double coset $[(g, e)]$. Eq. (4.2) shows that this map is defined correctly. It is onto since each double coset of $\mathrm{D}\left(G_{+}\right)$ is equal to a coset $[(g, e)]$ for some $g \in G$, because of (4.1). To show that it is injective assume that $\left[\left(g_{1}, e\right)\right]=\left[\left(g_{2}, e\right)\right]$. If

$$
\left(m_{1}^{\prime} l_{1}^{\prime} g_{1}\left(l_{1}^{\prime}\right)^{-1} m_{1}^{\prime \prime} k_{1}, \mathrm{p}_{+}\left(k_{1}\right)\right)=\left(m_{2}^{\prime} l_{2}^{\prime} g_{2}\left(l_{2}^{\prime}\right)^{-1} m_{2}^{\prime \prime} k_{2}, \mathrm{p}_{+}\left(k_{2}\right)\right)
$$

is an elment of this double coset (the notation is as in (4.2) ) then $k_{1}\left(k_{2}\right)^{-1} \in \Lambda_{1}$. The first components give immediately that the $G_{+}$orbits of $g_{1} \Lambda_{1} M_{+}$and $g_{2} \Lambda_{1} M_{+}$coincide.

In the special case of an $r$-matrix $r$ for which $\mathfrak{g}_{+} \supset \mathfrak{h}$ (see Remark 3.8), Proposition 4.1 is about the conjugation action of the parabolic subgroup $P_{+}$of $G$ on $G /\left(\Lambda_{1} N_{+}\right)$, where $N_{+}$is its unipotent radical.

\subsubsection{Relation to double cosets in $G$ and the Bruhat Lemma}

Let

$$
\Pi: \mathrm{D}\left(G_{+}\right) \cong G \times\left(\left(L_{1}^{\prime} A_{1}\right) / \Lambda_{1}\right) \rightarrow G .
$$

be the projection map on the first component of $\mathrm{D}\left(G_{+}\right)$. It is clear that

$$
\Pi([(g, \tilde{l})])=G_{+} g G_{+}, \forall g \in G, \tilde{l} \in L_{1}^{\prime} A_{1}
$$

which connects $i_{+}\left(G_{+}\right)$double cosets in $\mathrm{D}\left(G_{+}\right)$with $G_{+}$double cosets in $G$.

The group $G_{+}$differs from the parabolic subgroup $P_{+}$of $G$ just on a part of the maximal torus $H$ (see (3.14) $)$. This makes the description of the $G_{+}$double cosets of $G$ an easy consequence from the one for $P_{+}$double cosets. The latter is given by the Bruhat Lemma.

For any element $w \in W$ we will denote with $\dot{w}$ a representative of it in the normalizer $N(H)$ of $H$ in $G$. The conjugation and the adjoint actions of $\dot{w}$ on $G$ and $\mathfrak{g}$ will be denoted by $\operatorname{Ad}_{\dot{w}}$. 
Lemma 4.2 (Bruhat) Let $P_{1}$ and $P_{2}$ be two parabolic subgroups of $G$, each of which contains one of the Borel subgroups $B_{+}$or $B_{-}$. If $W_{1,2}$ denote the Weyl groups of their Levi factors $L_{1,2}$ (naturally embedded in $W$ ) then

$$
G=\sqcup P_{1} \dot{w} P_{2},
$$

with $w$ running over a set of representatives in $W$ of all cosets from $W_{1} \backslash W / W_{2}$.

After commuting $\dot{w}$ with the part $Z\left(L_{2}\right)^{\circ}$ of the torus $H \subset P_{2}$, one gets

$$
G=\sqcup P_{1} \dot{w}\left(L_{2}^{\prime} \ltimes N_{2}\right)
$$

where $N_{2}$ is the unipotent radical of $P_{2}$ and the disjoint union is over the same subset of $W$ as in (4.5).

The standard length function on $W$ will be denoted by $l($.$) . In the following Lemma we$ collect some facts for minimal length representatives of double cosets of the Weyl group $W$ which will be needed later.

Lemma 4.3 In the notation of Lemma 4.9:

1. Each double coset from $W_{1} \backslash W / W_{2}$ has a unique representative $w \in W$ of minimal length. It can be equivalently described as the representative $w \in W$ of the coset for which:

(*) For all positive roots $\alpha$ of $\operatorname{Lie}\left(L_{1}\right)$ and $\beta$ of $\operatorname{Lie}\left(L_{2}\right), w^{-1}(\alpha)$ and $w(\beta)$ are positive roots of $\mathfrak{g}$.

For any such $w \in W, \mathfrak{l}^{w}=\operatorname{Lie}\left(L_{1}\right) \cap \dot{w}^{-1}\left(\operatorname{Lie}\left(L_{2}\right)\right)$ is a reductive subalgebra of $\mathfrak{g}$ generated by $\mathfrak{h}$ and root spaces of simple roots of $\mathfrak{g}$. Its Weyl group will be denoted by $W^{w}$.

2. Any element $u \in W$ can be uniquely represented as

$$
u=w_{1} w w_{2}
$$

with $w, w_{1}$ being minimal length representatives in $W, W_{1}$ of cosets from $W_{1} \backslash W / W_{2}$, $W_{1} / W^{w}$ and $w_{2} \in W_{2}$. Its length is given by

$$
l(u)=l\left(w_{1}\right)+l(w)+l\left(w_{2}\right) .
$$

3. Let $P_{3}$ be a parabolic subgroup of $G$ such that $B_{ \pm} \subset P_{3} \subset P_{1}$ and $W_{3}$ be the Weyl group of its Levi factor. Then the set of minimal length representatives in $W$ for the cosets from $W_{3} \backslash W / W_{2}$ consists of the elements $u \in W$ whose representation from part 2 has $w_{2}=\mathrm{id}$ and $w_{1}-$ minimal length representative in $W$ of a coset from $W_{3} \backslash W_{1} / W^{w}$.

Proof. In the case of $P_{1}=B_{ \pm}$(i.e. $W_{1}$ trivial) the Lemma is proved in [15], Proposition 1.10. We will use this fact and will refer to it as the single coset version of the Lemma.

Parts 1 and 2: Fix a coset from $W_{1} \backslash W / W_{2}$. Chose a representative $w$ of it in $W$ having the property $(*)$. Any minimal length representative $w_{0}$ of the double coset has this property since otherwise $l\left(s_{1} w_{0}\right)<l\left(w_{0}\right)$ or $l\left(w_{0} s_{2}\right)<l\left(w_{0}\right)$ for some simple reflections $s_{i} \in W_{i}, i=1,2$.

It is clear that any element $u \in W_{1} w W_{2}$ can be represented in the form (4.6) with $w_{i} \in W_{i}$ as in part 2 of the Lemma. We will show the uniqueness of this representation and prove (4.7). The latter implies the statement of part 1. 
Since $w_{1} \in W_{1}$ is the minimal length representative of a coset from $W_{1} / W^{w}$, it has the property that $w_{1}(\alpha)$ is a positive root of $\operatorname{Lie}\left(L_{1}\right)$ for any positive root $\alpha$ of $\mathfrak{l}^{w}$. This combined with the definition of $w$ implies that $w_{1} w$ is a minimal length representative in $W$ of the coset $w_{1} w W_{2}$. (Here we also use the fact that $w_{1}$ maps positive roots of $\mathfrak{g}$ that are not roots of $\operatorname{Lie}\left(L_{1}\right)$ into positive roots of $\mathfrak{g}$.) The single coset version of the Lemma applied to the coset $w_{1} w W_{2}$ implies that $w_{2}$ is determined uniquely from $u$ and therefore so is $w_{1}$.

The single coset version of (4.7) for the cosets $w_{1} w W_{2} \in W / W_{2}$ and $w_{1} W^{w} \in W_{1} / W^{w}$ with minimal representatives $w_{1} w$ and $w_{1}$ gives

$$
\begin{aligned}
& l\left(w_{1} w w_{2}\right)=l\left(w_{1} w\right)+l\left(w_{2}\right) \\
& l\left(w_{1} w\right)=l\left(w_{1}\right)+l(w)
\end{aligned}
$$

which together imply (4.7).

Part 3: Part 1 implies that the elements of $W$ listed in part 3 are minimal length representatives for cosets from $W_{1} \backslash W / W_{2}$. In the opposite direction, any minimal representative $u=w_{1} w w_{2} \in W$ written as in part 2 should have $w_{2}=\mathrm{id}$ and $w_{1}(\alpha)$ should be positive root of $\operatorname{Lie}\left(L_{1}\right)$ for any positive root $\alpha$ of $\mathfrak{l}^{w}$. This implies the stated property of $w_{2}$.

For the given $r$-matrix denote with $W_{1,2}$ the Weyl groups of the Levi factors $L_{1,2}$ of $P_{ \pm}$. The Bruhat Lemma and (4.4) imply that any $i_{+}\left(G_{+}\right)$double coset in $\mathrm{D}\left(G_{+}\right)$is equal to a coset $[(h \dot{w}, \tilde{l})]$ for some $h \in H, \tilde{l} \in\left(L_{1}^{\prime} A_{1}\right) / \Lambda_{1}$ and $w$ - minimal length representative in $W$ of a double coset from $W_{1} \backslash W / W_{1}$. Taking into account (4.1) we obtain:

Lemma 4.4 Each $G_{+}$double coset of $\mathrm{D}(G)$ coincides with one of the cosets $[(l \dot{w}, e)]$ for $w$ - minimal representative in $W$ of a coset from $W_{1} \backslash W / W_{1}$ and $l \in L_{1}$.

\subsubsection{Main result}

Let $V \subset W$ denotes the set of minimal length representatives of the cosets from $W / W_{1}$. According to Lemma 4.3, each such coset has a unique representative in $V$.

For an element $v \in V$ denote by $\mathfrak{g}^{v}$ the largest Lie subalgebra of $\mathfrak{l}_{1}$ that is stable under $\operatorname{Ad}_{\dot{v}}$. It does not depend on the choice of representative of $v$ in $N(H)$ and can also be defined by:

$$
\mathfrak{g}^{v}=\operatorname{span}\left\{h, \mathfrak{g}^{\alpha} \mid \text { for the roots } \alpha \text { of } \mathfrak{l}_{1} \text { such that } v^{n}(\alpha) \text { is a root of } \mathfrak{l}_{1} \forall \text { integer } n\right\} .
$$

The minimality condition on $v$ guarantees that $\mathfrak{g}^{v}$ is a reductive Lie subalgebra of $\mathfrak{g}$, generated by the Cartan subalgebra $\mathfrak{h}$ and root spaces of simple roots of $\mathfrak{g}$. This follows from the fact that for any positive root $\alpha$ of $\mathfrak{l}_{1}, v(\alpha)$ is a positive root of $\mathfrak{g}$ (cf. Lemma 1.3). Denote with $G^{v}$ the reductive subgroup of $G$ with Lie algebra $\mathfrak{g}^{v}$ and with $W^{v}$ its Weyl group. Clearly $G^{v}$ is stable under $\operatorname{Ad}_{\dot{v}}$.

The definition of $\mathfrak{g}^{v}$ combined with the minimality property of $v$ implies that for any positive root $\alpha$ of $\mathfrak{g}^{v}, v(\alpha)$ is again a positive root of $\mathfrak{g}^{v}$, and thus so is $v^{-1}(\alpha)$. From part 1 of Lemma 4.3 it follows that any $v \in V$ is the minimal length representative of the double coset $W^{v} v W_{1}$. 
The fact that $\operatorname{Ad}_{\dot{v}}$ preserves $G^{v}$ implies that it also preserves its derived group $\left(G^{v}\right)^{\prime}$ and the identity component of its center $Z\left(G^{v}\right)^{\circ}$. Thus we can define an action of $\left(G^{v}\right)^{\prime}$ on itself by

$$
T C_{g}^{\dot{v}}(f)=g^{-1} f \operatorname{Ad}_{\dot{v}}(g), g, f \in\left(G^{v}\right)^{\prime}
$$

which will be called twisted conjugation action.

The fact that $\Lambda_{1}$ is a subgroup of $Z\left(L_{1}^{\prime} A_{1}\right) \subset H$ implies $\Lambda_{1} \subset G^{v}$. Let $\Lambda_{1}^{v}$ be the subgroup of $\left(G^{v}\right)^{\prime} \times Z\left(G^{v}\right)^{\circ}$ which is the inverse image of $\Lambda_{1}$ under the multiplication map

$$
\left(G^{v}\right)^{\prime} \times Z\left(G^{v}\right)^{\circ} \rightarrow G^{v}=\left(G^{v}\right)^{\prime} Z\left(G^{v}\right)^{\circ} .
$$

Note that $\Lambda_{1}^{v} \subset Z\left(\left(G^{v}\right)^{\prime} \times Z\left(G^{v}\right)^{\circ}\right)$ and that each element of $\Lambda_{1}$ has finitely many preimages in $\Lambda_{1}^{v}$ (more precisely \# $\left.\left(G^{v}\right)^{\prime} \cap Z\left(G^{v}\right)^{\circ}\right)$.

Denote by $L^{v}$ the connected subgroup of $G^{v}$ with Lie algebra $\mathfrak{g}^{v} \cap\left(\mathfrak{l}_{1}^{\prime} \oplus \mathfrak{a}_{1}\right)$. Let $\operatorname{Cong}^{v}\left(L^{v}\right)$ be the subgroup of the abelian group $Z\left(G^{v}\right)^{\circ}$ defined by

$$
\operatorname{Cong}^{v}\left(L^{v}\right)=\left\{h^{-1} \operatorname{Ad}_{\dot{v}}(h) \mid h \in Z\left(L^{v}\right)^{\circ}\right\} .
$$

$H_{1}^{\text {ort }}$ and $\operatorname{Ad}_{\dot{v}}\left(H_{1}^{\text {ort }}\right)$ are also subgroups of $Z\left(G^{v}\right)^{\circ}$ since by the definition of $H_{1}^{\text {ort }}$, $H_{1}^{\text {ort }} \subset Z\left(L_{1}\right)^{\circ}$.

Theorem 4.5 The $i_{+}\left(G_{+}\right)$double cosets of $\mathrm{D}\left(G_{+}\right)$are classified by a minimal length representative $v$ in $W$ of a coset from $W / W_{1}$, an orbit of the twisted conjugation action (4.9) of $\left(G^{v}\right)^{\prime}$ on itself, and an element of the abelian group

$$
Z\left(G^{v}\right)^{\circ} /\left(\operatorname{Cong}^{v}\left(L^{v}\right) H_{1}^{\text {ort }} \operatorname{Ad}_{\dot{v}}\left(H_{1}^{\text {ort }}\right)\right)
$$

For a fixed $v$ this data has to be taken modulo the multiplication action of $\Lambda_{1}^{v} \subset Z\left(\left(G^{v}\right)^{\prime} \times Z\left(G^{v}\right)^{\circ}\right)$.

The dimension of the coset corresponding to the orbit through $f \in\left(G^{v}\right)^{\prime}$ is:

$\operatorname{dim} \mathfrak{g}_{+}-\operatorname{dim} \mathfrak{h}_{1}^{\text {ort }}+\operatorname{dim}\left(\mathfrak{l}_{1}^{\prime} \oplus \mathfrak{a}_{1}\right)-\operatorname{dim} \mathfrak{l}^{v}+l(v)+T C_{\left(G^{v}\right)^{\prime}}^{\dot{v}}(f)+\operatorname{dim} \operatorname{Cong}^{v}\left(L^{v}\right) H_{1}^{\text {ort }} \operatorname{Ad}_{\dot{v}}\left(H_{1}^{\text {ort }}\right)$.

(The described data corresponding to different choices of a representative in $N(H)$ of a fixed element $v \in W$ can be canonically identified.)

Proof. In the previous Subsection we showed that any $i_{+}\left(G_{+}\right)$double coset of $\mathrm{D}\left(G_{+}\right)$is equal to a coset $[(l \dot{w}, e))]$ for some $l \in L_{1}$ and $w$ minimal length representative in $W$ of a coset from $W_{1} \backslash W / W_{1}$.

Based on the elements $l$ and $\dot{w}$ we will define a sequence of reductive Lie subalgebras $\mathfrak{g}^{k}$ of $\mathfrak{g}$, elements $g^{k} \in \mathfrak{g}^{k}$, and $w^{k} \in W(k \geq 1)$. It starts with $\mathfrak{g}^{1}=\mathfrak{l}_{1}$ and $g^{1}=l, w^{1}=w$. The sequence will have the property that $\mathfrak{g}^{k}$ and

$$
\overline{\mathfrak{g}}^{k}=\operatorname{Ad}_{\left(\dot{w}^{k-1} \ldots \dot{w}^{1}\right)}\left(\mathfrak{g}^{k}\right)
$$

are reductive subalgebras of $\mathfrak{g}^{k-1} \subset \mathfrak{g}$ containing the Cartan subalgebra $\mathfrak{h}$ and generated by root spaces corresponding to simple roots of $\mathfrak{g}$. If $W^{k}$ and $\bar{W}^{k}$ are their Weyl groups considered as subgroups of $W^{k-1} \subset W, w^{k}$ will have the property that it is representative of minimal length of a coset from $W^{k} \backslash W^{k-1} / \bar{W}^{k}$. 
If $\operatorname{Ad}_{\left(\dot{w}^{k} \ldots \dot{w}^{1}\right)}$ does not preserve $\mathfrak{g}^{k}$ define $\mathfrak{g}^{k+1}$ by

$$
\mathfrak{g}^{k+1}=\mathfrak{g}^{k} \cap \operatorname{Ad}_{\left(\dot{w}^{k} \ldots \dot{w}^{1}\right)}^{-1}\left(\mathfrak{g}^{k}\right) .
$$

The minimality property of $w^{j}$ implies that $w^{k} \ldots w^{1}$ is the minimal length representative of a coset from $W^{k} \backslash W / W^{1}$ (see part 3 of Lemma 4.3) and therefore for any positive root $\alpha$ of $\mathfrak{g}^{k},\left(w^{k} \ldots w^{1}\right)(\alpha)$ and $\left(w^{k} \ldots w^{1}\right)^{-1}(\alpha)$ are positive roots of $\mathfrak{g}$. From this we get that both $\mathfrak{g}^{k+1}$ and $\overline{\mathfrak{g}}^{k+1}=\operatorname{Ad}_{\left(\dot{w}^{k} \ldots \dot{w}^{1}\right)}\left(\mathfrak{g}^{k+1}\right)$ are generated by $\mathfrak{h}$ and root spaces of simple roots of $\mathfrak{g}$.

Let $\mathfrak{n}_{+}^{k+1}$ and $\overline{\mathfrak{n}}_{+}^{k+1}$ be the two nilpotent subalgebras of $\mathfrak{g}^{k}$ spanned by the root spaces of positive roots of $\mathfrak{g}^{k}$ that are not roots of $\mathfrak{g}^{k+1}$ and $\overline{\mathfrak{g}}^{k+1}$ respectively.

The connected subgroups of $G$ with Lie algebras $\mathfrak{g}^{k+1}, \overline{\mathfrak{g}}^{k+1}, \mathfrak{n}_{+}^{k+1}$, and $\overline{\mathfrak{n}}_{+}^{k+1}$ will be denoted by $G^{k+1}, \bar{G}^{k+1}, N_{+}^{k+1}$, and $\bar{N}_{+}^{k+1}$. They give rise to two parabolic subgroups $G^{k+1} \ltimes N_{+}^{k+1}$ and $\bar{G}^{k+1} \ltimes \bar{N}_{+}^{k+1}$ of $G^{k}$. To define recursively $g^{k+1}$ and $w^{k+1}$ from $g^{k}$ and $w^{k}$ we apply the Bruhat Lemma for the above parabolic subgroups of $G^{k}$. We get that $g^{k} \in G^{k}$ can be represented as

$$
g^{k}=n^{\prime} g^{\prime} \dot{w}^{k+1} g^{\prime \prime} n^{\prime \prime}
$$

for some $w^{k+1} \in W^{k+1}$ that is the representative of minimal length of a coset from $W^{k+1} \backslash W^{k} / \bar{W}^{k+1}$ and some $g^{\prime} \in G^{k+1}, g^{\prime \prime} \in \bar{G}^{k+1}, n^{\prime} \in N_{+}^{k+1}, n^{\prime \prime} \in \bar{N}_{+}^{k+1}$. Eq. (4.14) is the defining relation for $w_{k+1}$ and $g^{k+1}$ is defined by

$$
g^{k+1}=\left(\operatorname{Ad}_{\left(\dot{w}^{k} \ldots \dot{w}^{1}\right)}^{-1}\left(g^{\prime \prime}\right)^{-1}\right) g^{\prime}
$$

Clearly the described process continues for finitely many steps $k=1, \ldots, K$ and at the end

$$
\operatorname{Ad}_{\left(\dot{w}^{K} \ldots \dot{w}^{1}\right)}\left(\mathfrak{g}^{K}\right)=\overline{\mathfrak{g}}^{K}=\mathfrak{g}^{K} .
$$

Again due to part 3 of Lemma 4.3 the minimality properties of $w^{1}, \ldots, w^{K}$ add up to

$$
v:=\left(w^{K} \ldots w^{1}\right) \in V .
$$

Further we will use the representative $\dot{v}=\dot{w}^{K} \ldots \dot{w}^{1}$ of $v$ in $N(H)$.

Observe that

$$
\mathfrak{g}^{K}=\mathfrak{g}^{v}
$$

Indeed, in view of $(4.16)$ it is sufficient to prove that $\mathfrak{g}^{v} \subset \mathfrak{g}^{K}$. One proves by induction that $\mathfrak{g}^{v} \subset \mathfrak{g}^{k}$ for $k=1, \ldots, K$. The step from $k$ to $k+1$ follows from the definition (4.13) of $\mathfrak{g}^{k+1}$ and the fact that $\left(w^{K} \ldots w^{k+2}\right) \in W^{k+1}$.

Thus we have $G^{K}=G^{v}=\left(G^{v}\right)^{\prime} Z\left(G^{v}\right)^{\circ}$ and let

$$
g^{K}=f^{K} c^{K}
$$

for some $f^{K} \in\left(G^{v}\right)^{\prime}$ and $c^{K} \in Z\left(G^{v}\right)^{\circ}$ defined uniquely modulo multiplication of $f^{K}$ and division of $c^{K}$ by an element of the finite group $\left(G^{v}\right)^{\prime} \cap Z\left(G^{v}\right)^{\circ}$. The statement from Theorem 4.5 about the set of $i_{+}\left(G_{+}\right)$double cosets follows from the following two Lemmas. 
Lemma 4.6 In the above notation $[(l \dot{w}, e)]=\left[\left(g^{K} \dot{v}, e\right)\right]$ and two double cosets as the one in the right hand side coincide if and only if they are related to one and the same $v \in V$, the twisted conjugation orbits $T C_{\left(G^{v}\right)^{\prime}}^{\dot{v}}\left(f^{K}\right)$ that correspond to them and the images of the their elements $c^{K}$ in the factor group (4.10) are equal modulo the multiplication action of $\Lambda_{1}^{v}$ on $\left(G^{v}\right)^{\prime} \times Z\left(G^{v}\right)^{\circ}$.

For Theorem 4.5 we associate to the double coset $[(l \dot{w}, e)]=\left[\left(g^{K} \dot{v}, e\right)\right]$ the $T C^{\dot{v}}$ orbit of $f^{K}$ and the image of $c^{K}$ in the abelian factor group (4.10) (recall eq. (4.18)).

Lemma 4.7 Any element of $V$ can be obtained in a unique way as a product $\left(w^{K} \ldots w^{1}\right)$ through the described iterative procedure.

We will need some more notation: let $\mathfrak{n}_{-}^{k}$ denote the nilpotent subalgebras of $\mathfrak{g}^{k-1}$ spanned by root spaces of negative roots of $\mathfrak{g}^{k-1}$ that are not roots of $\mathfrak{g}^{k}$. For convenience we set $\mathfrak{g}^{0}=\mathfrak{g}$ and define $\mathfrak{n}_{ \pm}^{k}$ and $\overline{\mathfrak{n}}_{+}^{k}$ for $k=1$ also.

Let also $\mathfrak{l}^{k}=\mathfrak{g}^{k} \cap\left(\mathfrak{l}_{1} \oplus \mathfrak{a}_{1}\right)$ and denote by $L^{k}$ the corresponding connected subgroup of $G$. Note that $L^{K}=L^{v}$.

Proof of Lemma 4.0. Our strategy in dealing with the double coset $[(l \dot{w}, e)]=\left[\left(g^{1} \dot{w}^{1}, e\right)\right]$ is to commute the right factor $i\left(G_{+}\right)$with $\dot{w}=\dot{w}^{1}$, splitting it according to how the result is related to the left factor $i_{+}\left(G_{+}\right)$. Then we continue inductively, following the described procedure. Note that $\mathfrak{l}^{1}=\mathfrak{l}_{1}^{\prime} \oplus \mathfrak{a}_{1}$ and $\mathfrak{m}_{+}$admit the following decompositions as direct sum of linear spaces, each term of which is a Lie subalgebra:

$$
\begin{aligned}
& \mathfrak{l}^{1}=\left(\mathfrak{l}^{1} \cap \operatorname{Ad}_{\dot{w}^{1}}^{-1}\left(\mathfrak{n}_{+}^{1}\right)\right) \dot{+}\left(\mathfrak{l}^{1} \cap \operatorname{Ad}_{\dot{w}^{1}}^{-1}\left(\mathfrak{g}^{1}\right)\right) \dot{+}\left(\mathfrak{l}^{1} \cap \operatorname{Ad}_{\dot{w}^{1}}^{-1}\left(\mathfrak{n}_{-}^{1}\right)\right), \\
& \mathfrak{m}_{+}=\left(\overline{\mathfrak{n}}_{+}^{1} \cap \operatorname{Ad}_{\dot{w}^{1}}^{-1}\left(\mathfrak{n}_{+}^{1}\right)\right) \dot{+}\left(\mathfrak{m}_{+} \cap \operatorname{Ad}_{\dot{w}^{1}}^{-1}\left(\mathfrak{g}^{1}\right)\right) \dot{+}\left(\overline{\mathfrak{n}}_{+}^{1} \cap \operatorname{Ad}_{\dot{w}^{1}}^{-1}\left(\mathfrak{n}_{-}^{1}\right)\right) .
\end{aligned}
$$

These formulas imply that the product

$$
\begin{aligned}
& \left(L^{1} \cap \operatorname{Ad}_{\dot{w}^{1}}^{-1}\left(N_{+}^{1}\right)\right)\left(\bar{N}_{+}^{1} \cap \operatorname{Ad}_{\dot{w}^{1}}^{-1}\left(N_{+}^{1}\right)\right)\left(L^{1} \cap \operatorname{Ad}_{\dot{w}^{1}}^{-1}\left(G^{1}\right)\right)\left(M_{+} \cap \operatorname{Ad}_{\dot{w}^{1}}^{-1}\left(G^{1}\right)\right) \times \\
& \times\left(L^{1} \cap \operatorname{Ad}_{\dot{w}^{1}}^{-1}\left(N_{-}^{1}\right)\right)\left(\bar{N}_{+}^{1} \cap \operatorname{Ad}_{\dot{w}^{1}}^{-1}\left(N_{-}^{1}\right)\right)
\end{aligned}
$$

is a dense, open subset of $G_{+}$, because the linear span of the Lie algebras of all factors is $\mathfrak{g}_{+}$. Although $\overline{\mathfrak{n}}_{+}^{1}=\mathfrak{n}_{+}^{1}\left(=\mathfrak{n}_{+}\right)$and $\bar{N}_{+}^{1}=N_{+}^{1}\left(=N_{+}\right)$, we use both notations since this is how the groups $\bar{N}_{+}^{k} \neq N_{+}^{k}$ appear at the next steps $k \geq 2$.

From this it follows that the double coset $[(l \dot{w}, e)]=\left[\left(g^{1} \dot{w}^{1}, e\right)\right]$ of $\mathrm{D}\left(G_{+}\right)$has a dense, open subset consisting of elements of the form

$$
\left(m^{\prime} l^{\prime} g^{1} \dot{w}^{1} l_{+}^{\prime \prime} n_{+}^{\prime \prime} l_{*}^{\prime \prime} m_{*}^{\prime \prime} l_{-}^{\prime \prime} n_{-}^{\prime \prime}, \mathrm{p}_{+}\left(l^{\prime}\right) \mathrm{p}_{+}\left(l_{+}^{\prime \prime}\right) \mathrm{p}_{+}\left(l_{*}^{\prime \prime}\right) \mathrm{p}_{+}\left(l_{-}^{\prime \prime}\right)\right)
$$

for some arbitrary elements $l_{+}^{\prime \prime}, n_{+}^{\prime \prime}, l_{*}^{\prime \prime}, m_{*}^{\prime \prime}, l_{-}^{\prime \prime}$, and $n_{-}^{\prime \prime}$ of the groups of the product (4.21) (taken in the same order). Note that after commuting the terms $l_{+}^{\prime \prime}$ and $n_{+}^{\prime \prime}$ with $\dot{w}^{1}$ in the first component, they will be absorbed by the term $m^{\prime} \in M_{+}$.

The group $L^{1}$ by itself has a dense, open subset

$$
\left(L^{1} \cap \operatorname{Ad}_{\dot{w}^{1}}^{-1}\left(N_{-}^{1}\right)\right)\left(L^{1} \cap \operatorname{Ad}_{\dot{w}^{1}}^{-1}\left(G^{1}\right)\right)\left(L^{1} \cap \operatorname{Ad}_{\dot{w}^{1}}^{-1}\left(N_{+}^{1}\right)\right)
$$


If the term $l^{\prime}$ from (4.22) is taken in it, after some manipulations one shows that the coset $\left[\left(g^{1} \dot{w}^{1}, e\right)\right]$ has the following dense, open subset

$$
\begin{aligned}
& \left(N_{+}^{1}, e\right)\left(\left(\mathrm{id}, \mathrm{p}_{+}\right)\left(N_{-}^{2}\right)\right)\left(e, \mathrm{p}_{+}\left(N_{+}^{2}\right)\right) \times \\
& \times\left(H_{1}^{\text {ort }} N_{+}^{2}, e\right)\left(\left(\mathrm{id}, \mathrm{p}_{+}\right)\left(L^{2}\right)\right)\left(g^{1}, e\right)\left(\left(\operatorname{Ad}_{\dot{w}^{1}}, \mathrm{p}_{+}\right)\left(L^{2}\right)\right)\left(\bar{N}_{+}^{2} \operatorname{Ad}_{\dot{w}^{1}}\left(H_{1}^{\text {ort }}\right), e\right) \times \\
& \times\left(\left(\mathrm{id}, \mathrm{p}_{+} \operatorname{Ad}_{\dot{w}^{1}}^{-1}\right)\left(\operatorname{Ad}_{\dot{w}^{1}}\left(L^{1}\right) \cap N_{-}^{1}\right)\right)\left(\operatorname{Ad}_{\dot{w}^{1}}\left(\bar{N}_{+}^{1}\right) \cap N_{-}^{1}, e\right)\left(\dot{w}^{1}, e\right) .
\end{aligned}
$$

Here for a subgroup $R_{1}$ of $R_{2}$, and two endomorphisms $\sigma_{1}, \sigma_{2}$ of $R_{2},\left(\sigma_{1}, \sigma_{2}\right)\left(R_{1}\right)$ denotes the image of the composition of the diagonal embedding of $R_{1}$ in $R_{2} \times R_{2}$ composed with $\sigma_{1} \times \sigma_{2}$.

An important point in the derivation of formula (4.23) is that $H_{1}^{\text {ort }}$ commutes with $L^{1}=L_{1}^{\prime} A_{1}$ (see (3.5)) and thus with $N_{-}^{2} \subset L^{1}$. Later this will be used for all groups $N_{-}^{k} \subset L^{1}, k=2, \ldots, K$.

Note that $\Lambda_{1} \subset Z\left(L_{1}^{\prime} A_{1}\right) \subset H$ implies $\Lambda_{1} \subset L^{k}(\forall k)$. The product on the second line of $(4.23)$ is the double - left $\left(H_{1}^{\text {ort }} N_{+}^{2}\right) L^{2}$ and right $\operatorname{Ad}_{\dot{w}^{1}}\left(L^{2}\right)\left(\bar{N}_{+}^{2} \operatorname{Ad}_{\dot{w}^{1}}\left(H_{1}^{\text {ort }}\right)\right)$ coset of $\left(g^{1}, e\right)$ in $G^{1} \times\left(L^{2} / \Lambda_{1}\right)$ where the two groups are embedded in $G^{1} \times\left(L^{2} / \Lambda_{1}\right)$ as it is shown in the formula. Using the representation (4.14) of $g^{1}$ and (4.1) one easily shows that $\left[\left(g^{1} \dot{w}^{1}, e\right)\right]=\left[\left(g^{2} \dot{w}^{2}, e\right)\right]$ and procceeds by induction on $k$.

This procedure proves that $[(l \dot{w}, e)]=\left[\left(g^{K} \dot{v}, e\right)\right]$ (recall $\left.(4.17)\right)$. To prove the rest of Lemma 1.6, let us rearrange the coset from the last step similarly to (4.2):

$$
\begin{aligned}
& \left(H_{1}^{\text {ort }} N_{+}^{K}, e\right)\left(\left(\mathrm{id}, \mathrm{p}_{+}\right)\left(L^{K}\right)\right)\left(g^{K}, e\right)\left(\left(\operatorname{Ad}_{\dot{v}}, \mathrm{p}_{+}\right)\left(L^{K}\right)\right)\left(\bar{N}_{+}^{K} \operatorname{Ad}_{\dot{v}}\left(H_{1}^{\text {ort }}\right), e\right) \\
& =\left(N_{+}^{K}, e\right)\left(\left(\mathrm{id}, \mathrm{p}_{+}\right)\left(L^{K}\right)\right)\left(\widetilde{T C}_{L^{v} \times H_{1}^{\text {ort }} \times H_{1}^{\text {ort }}}\left(g^{K}\right), e\right)
\end{aligned}
$$

where we used that $\bar{N}_{+}^{K}=N_{+}^{K}$ normalizes $\bar{G}^{K}=G^{K}$. The action $\widetilde{T C}{ }^{\dot{v}}$ of $L^{v} \times H_{1}^{\text {ort }} \times H_{1}^{\text {ort }}$ on $G^{v}$ is defined by

$$
\widetilde{T C}_{\left(l, h^{\prime}, h^{\prime \prime}\right)}^{\dot{v}}(g)=h^{\prime} l^{-1} g \operatorname{Ad}_{\dot{v}}\left(l h^{\prime \prime}\right), \text { for } g \in G^{v}, l \in L^{v}, h^{\prime}, h^{\prime \prime} \in H_{1}^{\text {ort }} .
$$

In $(4.25)$ the term $i_{+}\left(H_{1}^{\text {ort }} L^{K}\right)$ is replaced by $i_{+}\left(H_{1}^{\text {ort }}\right) i_{+}\left(L^{K}\right)$ and $\left(\operatorname{Ad}_{i} \times \mathrm{id}\right)\left(i_{-}\left(L^{K} H_{1}^{\text {ort }}\right)\right)$ by $\left(\operatorname{Ad}_{\dot{v}} \times \mathrm{id}\right)\left(i_{-}\left(L^{K}\right) i_{-}\left(H_{1}^{\text {ort }}\right)\right)$. (The intersection $H_{1}^{\text {ort }} \cap L^{K}=H_{1}^{\text {ort }} \cap L_{1}^{\prime} A_{1}$ is nontrivial in general.)

In the case when $H_{1,2}^{\text {ort }}$ are trivial, the action 4.26 gives a simpler classification of the $i_{+}\left(G_{+}\right)$double cosets (see Corollary 4.8).

Using the decomposition (4.18) of $g^{K}$ the middle set in (4.25) can be rewritten as

$$
\widetilde{T C}_{L^{v} \times H_{1}^{\text {ort }} \times H_{1}^{\text {ort }}}^{\dot{v}}\left(g^{K}\right)=T C_{\left(G^{v}\right)^{\prime}}^{\dot{v}}\left(f^{K}\right)\left(c^{K} \operatorname{Cong}^{v}\left(L^{v}\right) H_{1}^{\text {ort }} \operatorname{Ad}_{\dot{v}}\left(H_{1}^{\text {ort }}\right)\right) .
$$

The inductive argument shows that the coset $[(l \dot{w}, e)]$ has the following dense, open subset:

$$
\left(N_{+}^{1}, e\right)\left(\prod_{k=2}^{K} E^{k}\right)\left(\left(\mathrm{id}, \mathrm{p}_{+}\right)\left(L^{K}\right)\right) \times
$$




$$
\begin{aligned}
& \times\left(T C_{\left(G^{v}\right)^{\prime}}^{\dot{v}}\left(g^{K}\right)\left(c^{K} \operatorname{Cong}^{v}\left(L^{v}\right) H_{1}^{\text {ort }} \operatorname{Ad}_{\dot{v}}\left(H_{1}^{\text {ort }}\right)\right), e\right) \times \\
& \times\left(\prod_{k=K-1}^{1} F_{k}\right)\left(\dot{w}^{K} \ldots \dot{w}^{1}, e\right) .
\end{aligned}
$$

where the subsets $E^{k}$ and $F^{k}$ of $G$ are defined by

$$
E_{k}=\left(\left(\mathrm{id}, \mathrm{p}_{+}\right)\left(N_{-}^{k}\right)\right)\left(N_{+}^{k} \times \mathrm{p}_{+}\left(N_{+}^{k}\right)\right)
$$

and

$$
\begin{aligned}
F_{k}= & \left(\left(\operatorname{Ad}_{\left(\dot{w}^{K} \ldots \dot{w}^{k}\right)}, \operatorname{Ad}_{\left(\dot{w}^{K} \ldots \dot{w}^{k+1}\right)} \mathrm{p}_{+}\right)\left(L^{k} \cap \operatorname{Ad}_{\dot{w}^{k}}^{-1}\left(N_{-}^{k}\right)\right)\right) \times \\
& \times\left(\operatorname{Ad}_{\left(w^{K} \ldots w^{k+1}\right)}\left(\operatorname{Ad}_{\dot{w}^{k}}\left(\bar{N}_{+}^{k}\right) \cap N_{-}^{k}\right), e\right) .
\end{aligned}
$$

The order of the factors in the first product in $(4.28)$ is $k=2, \ldots, K$ and in the second one is $k=K-1, \ldots, 1$.

Note that $\operatorname{Ad}_{\dot{w}^{1}}\left(\overline{\mathfrak{n}}_{+}^{1}\right) \cap \mathfrak{n}_{-}^{1}$ and $\operatorname{Ad}_{\dot{w}^{1}}\left(\mathfrak{l}^{1}\right) \cap \mathfrak{n}_{-}^{1}$ are subalgebras of $\mathfrak{n}_{-}^{1}$ and thus do not intersect $\mathfrak{n}_{+}^{1}$ and $\mathfrak{g}^{1}$. Using this, the fact that $\mathrm{p}_{ \pm}$are one to one on any unipotent subgroup of $L_{1,2}^{\prime} A_{1,2}$, and the Bruhat Lemma we see that if two subsets of $G$ of the type (4.23) are equal, then they are related to one and the same $w^{1} \in W$ and the corresponding double cosets of $G^{1} \times\left(L^{2} / \Lambda_{1}\right)$ (on line two) coinside. To finish the proof of Lemma 4.6, assume that two double cosets of the type $\left[\left(g^{K} \dot{v}, e\right)\right]$ obtained from described procedure coincide. Then their dense, open subsets from (4.23) will have nontrivial intersection. Using the above argument repeatedly we get that the two cosets are related to one and the same elements $w^{K}, \ldots, w^{1} \in W$ (and thus the same $v \in V$ ) and the corresponding to them orbits $\widetilde{T C}_{L^{v} \times H_{1}^{\text {ort }} \times H_{1}^{\text {ort }}}^{\dot{v}}\left(g^{K}\right)$ in $G^{v}$ coincide. The last is equivalent to saying that the orbits $T C_{\left(G^{v}\right)^{\prime}}^{\dot{v}}\left(f^{K}\right)$ corresponding to the two double cosets and the images of $c^{K}$ in the factor group (4.10) are equal modulo the multiplication action of $\Lambda_{1}^{v}$ on $\left(G_{1}^{v}\right)^{\prime} \times Z\left(G^{v}\right)^{\circ}$.

Proof of Lemma 4.7 . Let $v^{0}$ be an arbitrary element of $V$. Decompose it as $v^{0}=v^{1} w^{1} u^{1}$ with $v^{1}, u^{1} \in W_{1}$ and $w^{1}$ the minimal length representative in $W$ of the double cosets $W_{1} v W_{1}$. Since $v^{0}$ is minimal length representative of a coset from $W / W_{1}$, using part 3 of Lemma 4.3 we get that $u_{1}$ is the identity element of $W_{1}$. Define two reductive Lie subalgebras $\mathfrak{g}^{2}$ and $\overline{\mathfrak{g}}^{2}$ of $\mathfrak{g}^{1}$ by (4.13), (4.12) with $k=1$ and let $W^{2}, \bar{W}^{2}$ be the corresponding subgroups of $W$. The minimality property of $v^{0}$ implies that for any positive root $\alpha^{1}$ of $\mathfrak{g}^{1}, v\left(\alpha^{1}\right)$ is a positive root of $\mathfrak{g}$. This means that for any positive root $\alpha^{2}$ of $\overline{\mathfrak{g}}^{2}, v^{1}\left(\alpha^{2}\right)$ is a positive root of $\mathfrak{g}^{1}$. Therefore $v^{1}$ is minimal length representative in $W^{1}$ of a coset in $W^{1} / \bar{W}^{2}$. Continuing by induction we define $w^{k}, v^{k}, \mathfrak{g}^{k}, \overline{\mathfrak{g}}^{k}, W^{k}, \bar{W}^{k}$. At some step $k=K+1$ we will have $\mathfrak{g}^{K+1}=\overline{\mathfrak{g}}^{K+1}$ which is the same as $\mathfrak{g}^{K}=\operatorname{Ad}_{\left(\dot{w}^{K} \ldots \dot{w}^{1}\right)}\left(\mathfrak{g}^{K}\right)$. As in the proof of Lemma 4.6 set

$$
v=w^{K} \ldots w^{1}
$$

and then

$$
v^{0}=v^{K} v .
$$

We already proved that $\mathfrak{g}^{v}=\mathfrak{g}^{K}$. From (4.29) it follows that $\mathfrak{g}^{v^{0}}=\mathfrak{g}^{K}$, so both $v$ and $v^{0}$ have the property that they are minimal length representatives of one and the same 
coset from $W^{K} \backslash W / W^{1}$. The uniqueness of minimal length representative implies $v^{0}=v$ (see part 1 of Lemma 4.3). Comparing this procedure with the one from the beginning of the proof of Theorem 4.5 completes the proof of the existence part of Lemma 4.6. The uniqueness part is obtained by repatedly applying part 1 of Lemma 4.3.

Proof of formula (4.11). The double coset $\left[\left(g^{K} \dot{v}, e\right)\right]$ can be considered as an orbit of an action of the $2 \operatorname{dim} \mathfrak{g}_{+}$dimensional group $G_{+} \times G_{+}$on $\mathrm{D}\left(G_{+}\right)$. At the $k$-th step the set

$$
\left(\bar{N}_{+}^{k} \cap \operatorname{Ad}_{\dot{w}^{k}}^{-1}\left(N_{+}^{k}\right), e\right)
$$

coming from the second factor is annihilated $(k=1, \ldots, K-1)$. Its dimension is

$$
\operatorname{dim} \mathfrak{n}_{+}^{k}-\operatorname{dim} \mathfrak{n}_{+}^{k+1}-l\left(w^{k}\right) .
$$

Using part 2 of Lemma 4.3 we obtain that the sum of the dimensions of the annihilated sets is

$$
\operatorname{dim} \mathfrak{n}_{+}-\sum_{k=1}^{K} l\left(w^{k}\right)=\operatorname{dim} \mathfrak{n}_{+}-l(v) .
$$

At the end one needs to subtract also the dimension of the stabilizer of $g^{K}$ of the action $\widetilde{T C}^{\dot{v}}$ of $L^{v} \times\left(H^{\text {ort }}\right)^{\times 2}$. Passing to dimensions of orbits and using formula (4.27) we see that

$\operatorname{dim} \operatorname{Stab}_{\widetilde{T C}} \dot{v}\left(g^{K}\right)=\operatorname{dim} \mathfrak{l}^{v}+2 \operatorname{dim} \mathfrak{h}_{1}^{\text {ort }}-T C_{\left(G^{v}\right)^{\prime}}^{\dot{v}}\left(f^{K}\right)-\operatorname{dim}\left(\operatorname{Cong}^{v}\left(L^{v}\right) H_{1}^{\text {ort }} \operatorname{Ad}_{\dot{v}}\left(H_{1}^{\text {ort }}\right)\right)$.

Formula (4.11) is obtained by combining the above results with the fact that two elements of the set (4.28) are equal if and only if they have equal terms in each factor of the product.

Identification of the data of Theorem 4.5 corresponding to different choices of a representative of $v \in V$ in $N(H)$ : If $\dot{v}$ and $\ddot{v}$ are two different representative of $v \in V$ in $N(H)$ then

$$
\ddot{v}=h \dot{v}, \text { for some } h \in H,
$$

since the centralizer of a maximal torus in a complex reductive group coincides with the torus. Let $h=h^{v} h^{\circ}$ for some $h^{v} \in\left(G^{v}\right)^{\prime}$ and $h^{\circ} \in Z\left(G^{v}\right)^{\circ}$. A direct computation shows that the right multiplication by $h^{v}$ in $\left(G^{v}\right)^{\prime}$ maps $T C^{\ddot{v}}$ orbits to $T C^{\dot{v}}$ orbits:

$$
T C^{\ddot{v}}\left(g h^{v}\right)=T C^{\dot{v}}(g) h^{v}, \text { for all } g \in G^{v} .
$$

Since $\Lambda_{1}^{v}$ is in the center of $\left(G^{v}\right)^{\prime} \times Z\left(G^{v}\right)^{\circ}$ the right multiplication of $h^{v}$ on the first component of $\left(G^{v}\right)^{\prime} \times Z\left(G^{v}\right)^{\circ}$ commutes with the regular action of $\Lambda_{1}^{v}$. This identifies the data in Theorem 4.5 corresponding to two different choices of representatives $\dot{v}$ and $\ddot{v}$ of $v$ in $N(H)$.

Corollary 4.8 If the Poisson structure on $G$ comes from an $r$-matrix $r$ for which $\mathfrak{g}_{+} \supset \mathfrak{h}$, the $i_{+}\left(G_{+}\right)$double cosets of $\mathrm{D}\left(G_{+}\right)$are classified by a minimal length representative $v$ of 
a coset in $W / W_{1}$ and an orbit of the twisted conjugation action of $G^{v}$ on $G^{v} / \Lambda_{1}$ defined by

$$
\widetilde{T C}_{g}^{\dot{v}}(f)=g^{-1} f \operatorname{Ad}_{\dot{v}}(g) ; g \in G^{v}, f \in G^{v} / \Lambda_{1} .
$$

The dimension of the coset corresponding to the $\widetilde{T C}^{\dot{v}}$ orbit through $f \in G^{v} / \Lambda_{1}$ is

$$
\operatorname{dim} \mathfrak{g}_{+}+\operatorname{dim} \mathfrak{l}_{1}-\operatorname{dim} \mathfrak{l}^{v}+l(v)+\widetilde{T C}_{G^{v}}^{\dot{v}}(f) .
$$

The action (4.30) is the push down of the twisted conjugation action (4.26) of $G^{v}$ on itself to the factor group $G^{v} / \Lambda_{1}$. It is correctly defined since $\Lambda_{1} \subset Z\left(G^{v}\right)$.

Proof. In the considered case the groups $H_{1,2}^{\text {ort }}$ are trivial and $L^{v}=G^{v}$ (see Remark 3.8). The statement follows directly from the proof of Theorem 4.5, in particular (4.25).

Example 4.9 The simplest coset in $W / W_{1}$ is $W_{1}$, whose minimal length representative in $W$ is the identity element id of $W$. In this example we discuss the $i_{+}\left(G_{+}\right)$double cosets corresponding to it. We will assume that id is represented in $N(H)$ by the identity element $e$ of $G$. The group $L^{\text {id }}$ is simply $L_{1}^{\prime} A_{1}$ and for any coset of this type the inductive procedure of the proof of Theorem 4.5 has only one step, i.e. $K=1$. In the product (4.21) all groups except the second, the third, and the forth are trivial. Besides this the full group $G_{+}$is represented as

$$
G_{+}=N_{+}\left(L_{1}^{\prime} A_{1}\right) H_{1}^{\text {ort }}
$$

not just a dense subset of it. (This is nothing but (3.14).) The expression (4.28) reduces to:

$$
[(f c, e)]=\left(N_{+}, e\right)\left(\left(\mathrm{id}, \mathrm{p}_{+}\right)\left(L_{1}^{\prime} A_{1}\right)\right)\left(T C_{L_{1}^{\prime}}^{e}(f)\left(c H_{1}^{\text {ort }}\right), e\right)
$$

for all $f \in L_{1}^{\prime}$ and $c \in Z\left(L_{1}\right)^{\circ}$. The action $T C^{e}$ is the usual conjugation action of $L_{1}^{\prime}$ on itself

$$
T C_{l}^{e}(f)=l^{-1} f l, l, f \in L_{1}^{\prime} .
$$

Note also that Cong ${ }^{\text {id }}\left(L^{\text {id }}\right) H_{1}^{\text {ort }} \operatorname{Ad}_{e}\left(H_{1}^{\text {ort }}\right)=H_{1}^{\text {ort }}$.

The subgroup $\Lambda_{1}^{\mathrm{id}}$ of $L_{1}^{\prime} \times Z\left(L_{1}\right)^{\circ}$ is the pull back of $\Lambda_{1}$ under the map $L_{1}^{\prime} \times Z\left(L_{1}\right)^{\circ} \rightarrow$ $L_{1}^{\prime} Z\left(L_{1}\right)^{\circ}=L_{1}$.

Thus the cosets corresponding to id $\in V$ are classified by the conjugation orbits on $L_{1}^{\prime}$ and the elements of the abelian group $Z\left(L_{1}\right)^{\circ} / H^{\text {ort }}$ taken modulo the multiplication action of $\Lambda_{1}^{\text {id }}$ on $L_{1}^{\prime} \times Z\left(L_{1}\right)^{\circ}$.

The dimension of the coset (4.32) is

$$
\operatorname{dim}\left(\mathfrak{g}_{+}\right)+\operatorname{dim}\left(T C_{L_{1}^{\prime}}^{e}(f)\right)
$$

which follows from (4.11) after some cancellations.

Example 4.10 Consider the case of the standard Poisson structure on a semisimple Lie group $G$ (see Examples 3.5 and 3.9).

The groups $W_{1,2}$ are trivial, so $W / W_{1}=W$. Any $i_{+}\left(B_{+}\right)$double coset of $D\left(B_{+}\right) \cong$ $G \times H$ is equal to a coset of the type $[(h \dot{w}, e)]$, with $w \in W, h \in H$. For all $w \in W$ the 
procedure from the proof of Theorem 4.5 again has only one step and $L^{w}=H$. All groups in the product (4.21) except the second, the third, and the sixth are trivial and it reduces to

$$
B_{+}=\left(N_{+} \cap \operatorname{Ad}_{\dot{w}}^{-1}\left(N_{+}\right)\right) H\left(N_{+} \cap \operatorname{Ad}_{\dot{w}}^{-1}\left(N_{-}\right)\right) .
$$

Since the full group $G_{+}=B_{+}$is represented in this way, using (4.28) we can get an explicit expression for any double coset of $\mathrm{D}\left(B_{+}\right)$:

$$
[(h \dot{w}, e)]=\left(N_{+}, e\right) d(H)\left(h \operatorname{Cong}^{w}(H), e\right)\left(\operatorname{Ad}_{\dot{w}}\left(N_{+}\right) \cap N_{-}, e\right)(\dot{w}, e), \forall w \in W, h \in H
$$

where

$$
\operatorname{Cong}^{w}(H)=\left\{\left(h^{\prime}\right)^{-1} \operatorname{Ad}_{\dot{w}}\left(h^{\prime}\right) \mid h^{\prime} \in H\right\}
$$

and $d(H)$ is the image of the diagonal embedding of $H$ in $G \times H$. The set $h \operatorname{Cong}^{w}(H)$ is the $\widetilde{T C}{ }_{H}^{w}$ orbit through $h$ from Corollary 4.8. The general twisted conjugation orbits in Theorem 4.5 and Corollary 4.8 can be considered as nonabelian analogs of this action.

The different cosets (4.33) are classified by the elements $w$ of the Weyl group and of the abelian group $H / \operatorname{Cong}^{w}(H)$ (or equivalently the orbits of the $\widetilde{T C} \dot{w}$ action of $H$ on itself). According to Theorem 1.10 this data classifies the symplectic leaves of $i_{-}\left(B_{-}\right) / \Sigma$.

Both (4.33) and (4.31) give

$$
\operatorname{dim}[(h \dot{w}, e)]=\operatorname{dim} \mathfrak{b}_{+}+l(w)+\operatorname{dim}\left(\operatorname{Cong}^{w}(H)\right) .
$$

The above description of $i\left(B_{+}\right)$double cosets of $\mathrm{D}\left(B_{+}\right)$(and symplectic leaves of $\left.i_{-}\left(B_{-}\right) / \Sigma_{-}\right)$for the standard Poisson-Lie structure on $G$ agrees with the results in [8, 13].

Example 4.11 The Cremmer-Gervais Poisson structure on $S L(n+1)$ was reviewed in Examples 3.6 and 3.10. In this case $W \cong S^{n+1}$ and $W_{i}$ can be identified with the subgroups $S_{i}^{n+1}$ of $S^{n+1}$ that permute the first and the last $n$ letters. Denote by $H$ the maximal torus of $S L(n+1)$ consisting of diagonal matrices. We will assume that any element of $S^{n+1}$ is represented in the normalizer of $H$ by a multiple of the corresponding standard permutation matrix. Any minimal length representative $\sigma$ in $S^{n+1}$ of a coset from $S^{n+1} / S_{1}^{n}$ should have the property that $\sigma(1), \ldots, \sigma(n)$ is an increasing sequence (see part 1 of Lemma 4.3). From this one finds that the set of such representatives consists of $\sigma_{1}^{j} \in S^{n+1}$, $j=0, \ldots, n$, defined by

$$
\sigma_{1}^{j}(p)=p, p=1, \ldots, j ; \sigma_{1}^{j}(p)=p+1, p=j+1, \ldots, n ; \sigma_{1}^{j}(n+1)=j+1 .
$$

In a compact form, they are the permutations

$$
\sigma_{1}^{j}=((j+1)(j+2) \ldots(n+1)) .
$$

The groups $G^{\dot{\sigma}_{1}^{j}}$ are

$G^{\dot{\sigma}_{1}^{j}}=\left\{\operatorname{diag}\left(A, a_{j+1}, \ldots, a_{n+1}\right) \mid A \in G L(j), a_{p} \in \mathbb{C} ; \operatorname{det}(A) a_{j+1} \ldots a_{n+1}=1\right\} \subset S L(n+1)$.

The twisted conjugation action of $G^{\sigma_{1}^{j}}$ on itself is given by

$$
\begin{aligned}
& \widetilde{T C}_{\operatorname{diag}\left(A, a_{j+1}, \ldots, a_{n+1}\right)}^{\dot{\sigma}_{1}^{j}} \operatorname{diag}\left(B, b_{j+1}, \ldots, b_{n+1}\right)= \\
& \operatorname{diag}\left(A^{-1} B A, a_{j+1}^{-1} b_{j+1} a_{\mu(j+1)}, \ldots, a_{n+1}^{-1} b_{n+1} a_{\mu(n+1)}\right)
\end{aligned}
$$


where $\mu=\left(\sigma_{1}^{j}\right)^{-1}$. To each such orbit one can associate the conjugation orbit of $G L(j)$ trough $B$. The correspondence is bijective and the difference in their dimensions is $n-j$. Note also that $l\left(\sigma_{1}^{j}\right)=n-j$.

Thus in the case of the Cremmer-Gervais structure the $i_{+}\left(G_{+}\right)$double cosets of $\mathrm{D}\left(G_{+}\right)$, or in other words the symplectic leaves of $i_{-}\left(G_{-}\right) / \Sigma_{-}$(recall Theorem 1.10) are classified by a choice of a number $j=0, \ldots, n$ and a conjugation orbit on $G L(j)$. The dimension of the corresponding double coset is

$$
n(n+1)+(n-j)(n+j+1)+\operatorname{dim}\left\{\operatorname{Ad}_{A}(B) \mid A \in G L(j)\right\} .
$$

\subsection{Structure of the group $\Sigma_{-}=i_{+}\left(G_{+}\right) \cap i_{-}\left(G_{-}\right) \subset \mathrm{D}\left(G_{+}\right)$}

Denote

$$
\Sigma_{-}=i_{+}\left(G_{+}\right) \cap i_{-}\left(G_{-}\right) \subset \mathrm{D}\left(G_{+}\right) .
$$

It is a discrete subgroup of $\mathrm{D}\left(G_{+}\right)$since $\operatorname{Lie}\left(i_{+}\left(G_{+}\right)\right) \cap \operatorname{Lie}\left(i_{-}\left(G_{-}\right)\right)=0$.

Theorem 4.12 The group $\Sigma_{-}$is the intersection of the $i_{ \pm}$images of the tori $\left(L_{i}^{\prime} \cap H\right) A_{i} \subset$ $G_{ \pm}$in the maximal torus $H \times\left(\left(L_{1}^{\prime} \cap H\right) A_{1} / \Lambda_{1}\right)$ of the reductive group $G \times\left(L_{1}^{\prime} A_{1} / \Lambda_{1}\right) \cong$ $\mathrm{D}\left(G_{+}\right)$.

Proof. Using the recursive procedure from Subsect. 3.3 we will show that the statement holds for $G^{(k)}$, assuming its validity for $G^{(k+1)}$. Note that it is trivial for $G^{(\operatorname{ord} \tau)}$ since $G^{(\operatorname{ord} \tau)}=H$. Clearly we can restrict ourselves to the step from $G^{(1)}$ to $G=G^{(0)}$.

Let

$$
l_{i} \in L_{i}^{\prime} A_{i}, i=1,2 ; m_{ \pm} \in M_{ \pm}
$$

be such that

$$
\left(l_{1} m_{+}, \mathrm{p}_{+}\left(l_{1}\right)\right)=\left(m_{-} l_{2}, \Theta^{-1} \circ \mathrm{p}_{-}\left(l_{2}\right)\right) \in i_{+}\left(G_{+}\right) \cap i_{-}\left(G_{-}\right) .
$$

From the first components we get

$$
l_{1} m_{+}=m_{-} l_{2} \in\left(\left(L_{1}^{\prime} A_{1}\right) M_{+}\right) \cap\left(\left(L_{2}^{\prime} A_{2}\right) M_{-}\right) .
$$

Let $N_{1 \pm}^{(1)}$ denote the unipotent subgroups of $L_{1}^{\prime}$ generated by one parameter subgroups of positive / negative roots of $\mathfrak{l}_{1}^{\prime}$ that are not roots of $\mathfrak{l}_{2}^{\prime}$. Similarly we define $N_{2 \pm}^{(1)}$ as the unipotent subgroups of $L_{2}^{\prime}$ corresponding to roots of $\mathfrak{l}_{2}^{\prime}$ which are not roots of $\mathfrak{l}_{1}^{\prime}$. Using the standard fact that the intersection of the two parabolic subgroups $P_{+}$and $P_{-}$of $G$ is connected we get

$$
\begin{aligned}
& m_{+} \in H_{1}^{\text {ort }} \ltimes N_{2+}^{(1)}, \\
& m_{-} \in N_{1-}^{(1)} \rtimes H_{2}^{\text {ort }},
\end{aligned}
$$

and

$$
\begin{aligned}
& l_{1} \in N_{1-}^{(1)} \rtimes\left(\left(L_{1}^{\prime} \cap L_{2}\right) A_{1}\right), \\
& l_{2} \in\left(\left(L_{2}^{\prime} \cap L_{1}\right) A_{2}\right) \ltimes N_{2+}^{(1)} .
\end{aligned}
$$


Let $m_{+}=h_{1} n_{2+}$ and $l_{1}=n_{1-} l_{1}^{(1)}$ be the decompositions of $m_{+}$and $l_{1}$ according to the semidirect products (4.38) and (4.40). Eq. (4.37) implies that the decompositions of $m_{-}$and $l_{2}$ according to (4.39) and (4.41) should be $m_{-}=n_{1-} h_{2}, l_{2}=k_{2}^{(1)} n_{2+}$ for some $h_{2} \in H_{2}^{\text {ort }}$ and $k_{2}^{(1)} \in\left(L_{2}^{\prime} \cap L_{1}\right) A_{2}$ such that

$$
l_{1}^{(1)} h_{1}=h_{2} k_{2}^{(1)} .
$$

Observe that

$$
\begin{aligned}
& \left(L_{1}^{\prime} \cap L_{2}\right) A_{1}=L_{1}^{(1)}, N_{2+}^{(1)}=N_{+}^{(1)} \\
& \theta\left(\operatorname{Lie}\left(\left(L_{1}^{\prime} \cap L_{2}\right) A_{1}\right)\right)=\operatorname{Lie}\left(L_{2}^{(1)} A_{2}^{(1)}\right), \theta\left(\operatorname{Lie}\left(N_{1-}^{(1)}\right)\right)=\operatorname{Lie}\left(N_{-}^{(1)}\right) .
\end{aligned}
$$

Since $N_{-}^{(1)}$ is a unipotent subgroup of $L_{2}^{\prime} A_{2}$ and the kernel $\Lambda_{2}$ of the projection p - : $L_{2}^{\prime} A_{2} \rightarrow L_{2}^{\prime} A_{2} / \Lambda_{2}$ consists of semisimple elements, eqs. (4.44) imply the existence of elements $n_{-}^{(1)} \in N_{-}^{(1)}$ and $l_{2}^{(1)} \in\left(L^{(1)}\right)^{\prime} A^{(1)}$ such that

$$
\begin{aligned}
& \Theta \circ \mathrm{p}_{+}\left(n_{1-}\right)=\mathrm{p}_{-}\left(n_{-}^{(1)}\right), \\
& \Theta \circ \mathrm{p}_{+}\left(l_{1}^{(1)}\right)=\mathrm{p}_{-}\left(l_{2}^{(1)}\right) .
\end{aligned}
$$

Comparing the second components of $(4.36)$ we get $\Theta \circ \mathrm{p}_{+}\left(n_{1-} l_{1}^{(1)}\right)=\mathrm{p}_{-}\left(k_{2}^{(1)} n_{2+}\right)$. Combining this equation with (4.45) and (4.46) gives

$$
\mathrm{p}_{-}\left(n_{-}^{(1)} l_{2}^{(1)}\right)=\mathrm{p}_{-}\left(k_{2}^{(1)} n_{2+}\right) .
$$

By modifying $l_{2}^{(1)} \in\left(L^{(1)}\right)^{\prime} A^{(1)}$ with an element of $\Lambda_{2} \subset\left(L^{(1)}\right)^{\prime} A^{(1)}$ one can make it satisfy

$$
n_{-}^{(1)} l_{2}^{(1)}=k_{2}^{(1)} n_{2+}
$$

while keeping (4.46). Expressing $k_{2}^{(1)}$ in terms $l_{1}^{(1)}$ from (4.42) and substituting it in (4.47) gives

$$
h_{2} n_{-}^{(1)} l_{2}^{(1)}=l_{1}^{(1)} h_{1} n_{2+} .
$$

Because $l_{1}^{(1)} \in\left(L_{1}^{\prime} \cap L_{2}\right) A_{2}=L_{1}^{(1)}$ and $n_{2+} \in N_{2+}^{(1)}=N_{+}^{(1)}$ eqs. (4.48) and (4.46) imply that

$$
\left(l_{1}^{(1)} h_{1} n_{2+}, \mathrm{p}_{+}\left(l_{1}^{(1)}\right)\right)=\left(h_{2} n_{-}^{(1)} l_{2}^{(1)}, \Theta^{-1} \circ \mathrm{p}_{-}\left(l_{2}^{(1)}\right)\right) \in i_{+}^{(1)}\left(G_{+}^{(1)}\right) \cap i_{-}^{(1)}\left(G_{-}^{(1)}\right) \subset \mathrm{D}\left(G_{+}^{(1)}\right) .
$$

Using the inductive assumption for validity of the statement of the Lemma for $G^{(1)}$, we get that $l_{1}^{(1)} \in H, n_{2+}=e$ and therefore $\left(l_{1} m_{+}, l_{1}\right) \in H \times\left(\left(L_{1}^{\prime} \cap H\right) \times A_{1}\right)$.

As a consequence of Theorem 4.12 we also obtain that the $\Sigma_{-}-$groups associated to the Poisson-Lie groups $G, G^{(1)}, \ldots, G^{(\text {ord } \tau)}$ are naturally isomorphic.

From Theorem 4.12 one easily derives an explicit formula for $\Sigma_{-}$in terms of the lattices of kernels of exponential maps for certain tori. We will restrict ourselves to the case of an $r$-matrix for which $\mathfrak{h}_{i}^{\text {ort }}$ are trivial since in the general case the formula is too cumbersome.

Denote by ker and ker' the kernels of the exponential maps for the tori $H$ and $H / \Lambda_{2}$. 
Corollary 4.13 In the case when the $r$-matrix $r$ is such that $\mathfrak{g}_{+} \supset \mathfrak{h}$, the group $\Sigma_{-}$is isomorphic to

$$
\operatorname{ker}^{\prime} /\left(\operatorname{ker}^{\prime} \cap(1-\theta) \text { ker }\right) .
$$

If in addition the groups $\Lambda_{i}$ are trivial, then

$$
\Sigma_{-}=\{(h, h) \mid h \in H, \Theta(h)=h\} .
$$

One easily deduces from the assumption $\mathfrak{g}_{+} \supset \mathfrak{h}$ and the fact $\mathrm{D}\left(\mathfrak{g}_{+}\right)=i_{+}\left(\mathfrak{g}_{+}\right) \dot{+} i_{-}\left(\mathfrak{g}_{-}\right)$, that $1-\theta$ is a linear automorphism of $\mathfrak{h}$. This implies that $(1-\theta)^{-1} \operatorname{ker}^{\prime}$ is a lattice in $\mathfrak{h}$. The quotient (4.49) of the two lattices is not a finite group in general.

Proof of Corollary 4.13. According to Theorem $4.12 \Sigma_{-}$is the subgroup of $H \times\left(H / \Lambda_{1}\right)$ consisting of elements

$$
\left(l_{1}, \mathrm{p}_{+}\left(l_{1}\right)\right)=\left(l_{2}, \Theta^{-1} \circ \mathrm{p}_{-}\left(l_{2}\right)\right)
$$

for some $l_{i} \in H$. Let $l_{1}=\exp (x)$ for some $x \in \mathfrak{h}$. The second component of eq. (4.51) gives $l_{2}=\exp (\theta x) \lambda_{2}$ for some $\lambda_{2} \in \Lambda_{2}$. From the first component we obtain

$$
\exp ((1-\theta) x)=\lambda_{2} \in \Lambda_{2}
$$

and thus $x \in(1-\theta)^{-1}$ ker' $^{\prime}$. Taking the quotient

$$
(1-\theta)^{-1} \operatorname{ker}^{\prime} /\left((1-\theta)^{-1} \operatorname{ker}^{\prime} \cap \operatorname{ker}\right)
$$

cancels the elements $x \in \mathfrak{h}$ for which $\exp (x)=e$. The final answer is obtained after applying $(1-\theta)$ to 4.52$)$.

Eq. (4.50) follows directly from (4.51).

Example 4.14 Consider the case of the standard Poisson structure on a complex semisimple Lie group $G$. Then $\Lambda_{2}$ is trivial and ker $^{\prime}=$ ker . Corollary 4.13 combined with the facts that the Cayley transform $\theta: \mathfrak{h} \rightarrow \mathfrak{h}$ is $-\left.\mathrm{id}\right|_{\mathfrak{h}}$ and $\Lambda_{2}$ is trivial gives

$$
\Sigma_{-} \cong \operatorname{ker} /(2 \text { ker }) \cong \mathbb{Z}^{\text {rankg }} /\left(2 \mathbb{Z}^{\text {rankg }}\right) \cong \mathbb{Z}_{2}^{\text {rankg }}
$$

or equivalently

$$
\Sigma_{-}=\left\{(h, h) \mid h \in H, h^{2}=e\right\} .
$$

This coincides with the results in [8, 13].

Example 4.15 For the Cremmer-Gervais structure on $S L(n+1)$ the groups $\Lambda_{i}$ are trivial. The group $\Sigma_{-}$is explicitly given by

$$
\begin{aligned}
\Sigma_{-} & =\left\{(h, h) \mid h=\operatorname{diag}\left(h_{1}, \ldots, h_{n+1}\right) ; h_{i+1}=h_{i}, i=1, \ldots, n\right\} \\
& =\left\{(\epsilon E, \epsilon E) \mid \epsilon \in \mathbb{C}, \epsilon^{n+1}=1\right\} \cong \mathbb{Z}_{n+1}
\end{aligned}
$$

where $E$ denotes the identity matrix of size $n+1$. This can also be proved directly from (4.49) using the facts that the kernel of the exponential map on $\mathfrak{h}$ is

$$
\text { ker }=2 \pi i \mathbb{Z} \alpha_{1} \oplus \cdots \oplus 2 \pi i \mathbb{Z} \alpha_{n}
$$

and the Cayley transform on Lie algebra level acts by $\theta\left(\alpha_{j}\right)=\alpha_{j+1}$ for $j=1, \ldots, n-1$ and $\theta\left(\alpha_{n}\right)=-\alpha_{1}-\cdots-\alpha_{n}$. 


\subsection{Symplectic leaves of $G_{-}$}

In this Subsection we will summarize the results for the symplectic leaves of $G_{-}$and $i_{-}\left(G_{-}\right) / \Sigma_{-}$obtained by combining the results from Subsections 4.1, 4.2 and Theorems $1.9,1.10$.

Theorem 4.16 1. The symplectic leaves of $i_{-}\left(G_{-}\right) / \Sigma_{-}$are classified by the data from Theorem 4.5: An element $v \in V$, an orbit $T C^{\dot{v}}(f)$ in $\left(G^{v}\right)^{\prime}$, and an element of the abelian factor group $Z\left(G^{v}\right)^{\circ} /\left(\mathrm{Cong}^{v}\left(L^{v}\right) H_{1}^{\text {ort }} \mathrm{Ad}_{\dot{v}}\left(H_{1}^{\text {ort }}\right)\right)$, all taken modulo the multiplication action of $\Lambda_{1}^{v}$. The dimension of the leaf corresponding to this data is:

$$
\operatorname{dim}\left(\mathfrak{l}_{1}^{\prime} \oplus \mathfrak{a}_{1}\right)-\operatorname{dim} \mathfrak{l}^{v}-\operatorname{dim} \mathfrak{h}_{1}^{\text {ort }}+l(v)+\operatorname{dim} T C_{\left(G^{v}\right)^{\prime}}^{\dot{v}}(f)+\operatorname{dim} \operatorname{Cong}^{v}\left(L^{v}\right) H_{1}^{\text {ort }} \operatorname{Ad}_{\dot{v}}\left(H_{1}^{\text {ort }}\right) .
$$

2. The leaves of $G_{-}$are coverings of the leaves of $i_{-}\left(G_{-}\right) / \Sigma_{-}$under the projection map $G_{-} \rightarrow i_{-}\left(G_{-}\right) / \Sigma_{-}$. The discrete group $\Sigma_{-}$is characterized in Theorem 4.19. $G_{-}$.

In a special case Corollary 4.8 gives a simpler description of the symplectic leaves of

Corollary 4.17 In the case when $\mathfrak{g}_{+} \supset \mathfrak{h}$ the symplectic leaves of $i_{-}\left(G_{-}\right) / \Sigma_{-}$are in one to one correspondence with the elements $v \in V$ and the orbits of the action $\widetilde{T C}$ of $G^{v}$ on $G^{v} / \Lambda_{1}$. (see (4.30)). The dimension of the leaf corresponding to the orbit $\widetilde{T C}^{i}(f)$ is

$$
\operatorname{dim} \mathfrak{l}_{1}-\operatorname{dim} \mathfrak{g}^{v}+l(v)+\operatorname{dim} \widetilde{T C}_{G^{v}}^{\dot{v}}(f) .
$$

The group $\Sigma_{-}$is explicitly computed in Corollary 4.13.

Note also that the results from Sect. 4.1-4.2 give some information for the structure of the symplectic leaves of $i_{-}\left(G_{-}\right) / \Sigma_{-}$. In particular, the leaf corresponding to the choice of the data specified there has a dense, open subset which is the intersection of the set (4.28) (for $f^{K}=f, c^{K}=c$ ) with $i_{-}\left(G_{-}\right) i_{+}\left(G_{+}\right)$projected onto $i_{-}\left(G_{-}\right) i_{+}\left(G_{+}\right) / i_{+}\left(G_{+}\right)$.

\section{Symplectic leaves of $G$}

This Section describes the set of symplectic leaves of the full group $G$. The proofs of all results will only be sketched since they go along the lines of the proofs of the previous Section. We will restrict ourselves to the case when the derived subgroup $G^{\prime}$ of $G$ is simply connected. This is not an essential restriction since any factorizable reductive Poisson-Lie group $R$ has a covering which is a Poisson-Lie group $G$ of the considered type. The leaves of $R$ are simply projections of the ones of $G$.

The above assumption implies in particular that $L_{i}^{\prime}$ are also simply connected and the restriction of the map $\theta$ to $\mathfrak{l}_{1}^{\prime}$ can be lifted to an isomorphism

$$
\Theta^{\prime}: L_{1}^{\prime} \rightarrow L_{2}^{\prime}
$$

It clearly satisfies

$$
\Theta \circ \mathrm{p}_{+}(l)=\mathrm{p}_{-} \circ \Theta^{\prime}(l), \forall l \in L_{1}^{\prime} .
$$




\section{1 $G^{r}$ double cosets of $\mathrm{D}(G)$}

The double coset of an element $\left(g_{1}, g_{2}\right) \in G \times G \cong \mathrm{D}(G)$ will be denoted by $\left[\left(g_{1}, g_{2}\right)\right]$ :

$$
\left[\left(g_{1}, g_{2}\right)\right]:=G^{r}\left(g_{1}, g_{2}\right) G^{r} \subset G \times G \text {. }
$$

Similarly to (4.1) one has

$$
\left[\left(g_{1} l, g_{2}\right)\right]=\left[\left(g_{1}, g_{2} \Theta^{\prime}\left(l^{-1}\right)\right)\right],\left[\left(l g_{1}, g_{2}\right)\right]=\left[\left(g_{1}, \Theta^{\prime}\left(l^{-1}\right) g_{2}\right)\right], \forall l \in L_{1}^{\prime} .
$$

As in Subsect. 4.1.1 it can be shown that any $G^{r}$ double coset of $G \times G$ is equal to a coset of the form

$$
\left[\left(l_{1} \dot{w}_{1}, \dot{w}_{2} l_{2}\right)\right]
$$

where $l_{i} \in L_{i}$ and $w_{i}$ are representatives in $W$ of cosets from $W_{i} \backslash W / W_{i}, i=1,2$. For simplicity of notation it will be more convenient to have the cosets written as

$$
\left[\left(l_{1} \dot{w}_{1}, \dot{w}_{2}^{-1} l_{2}\right)\right]
$$

where $l_{i}$ and $w_{i}$ belong to the same sets as above.

\subsubsection{Description of the set of double cosets}

The map $\tau$ from the Belavin-Drinfeld triple $\left(\Gamma_{1}, \Gamma_{2}, \tau\right)$ associated to the $r$-matrix $r$ will be assumed to be extended to a linear bijection between the set of roots of $\mathfrak{l}_{1}$ and $\mathfrak{l}_{2}$.

Define the set $V$ as the subset of $W \times W$ consisting of pairs of elements $\left(v_{1}, v_{2}\right)$ of $W$ which are representatives of minimal length in $W$ of cosets from $W / W_{i}, i=1,2$. For each such pair $\left(v_{1}, v_{2}\right)$ let $\mathfrak{g}_{1}^{v_{1}, v_{2}}$ be the subalgebra of $\mathfrak{l}_{1}$ containing $\mathfrak{h}$ whose set of roots is stable under $v_{1} \tau^{-1} v_{2} \tau$ :

$$
\begin{aligned}
\mathfrak{g}_{1}^{v_{1}, v_{2}}=\operatorname{span}\left\{h, \mathfrak{g}^{\alpha} \mid \quad\right. & \text { for the roots } \alpha \text { of } \mathfrak{l}_{1} \text { such that }\left(v_{1} \tau^{-1} v_{2} \tau\right)^{n}(\alpha) \text { is well defined } \\
& \text { and is a root of } \left.\mathfrak{l}_{1} \forall \text { integer } n\right\} .
\end{aligned}
$$

Note that $v_{1} \tau^{-1} v_{2} \tau$ is not defined on all root spaces $\mathfrak{g}^{\alpha}$ of $\mathfrak{l}_{1}$ but only on those for which $v_{2} \tau(\alpha)$ is a root of $\mathfrak{l}_{2}$.

In this notation the maximal subalgebra of $\mathfrak{l}_{2}$ containing $\mathfrak{h}$ whose set of roots is preserved by $v_{2} \tau v_{1} \tau^{-1}$ is

$$
\mathfrak{g}_{2}^{v_{1}, v_{2}}=\operatorname{Ad}_{\dot{v}_{2}} \theta\left(\mathfrak{g}_{1}^{v_{1}, v_{2}}\right)^{\prime}+\mathfrak{h} .
$$

The minimality property of $v_{i}$ implies that $\mathfrak{g}_{i}^{v_{1}, v_{2}}$ are reductive Lie subalgebras of $\mathfrak{l}_{i}$ which are generated by $\mathfrak{h}$ and root spaces of simple roots of $\mathfrak{g}$. Denote by $G_{i}^{v_{1}, v_{2}}$ the connected subgroups of $G$ with Lie algebras $\mathfrak{g}_{i}^{v_{1}, v_{2}}$ and by $W_{i}^{v_{1}, v_{2}}$ their Weyl groups, $i=1,2$. Similarly to Sect. 4.1.2 one shows that $v_{i}$ are minimal length representatives for the double cosets $W_{i}^{v_{1}, v_{2}} v_{i} W_{i}$. Clearly $\operatorname{Ad}_{\dot{v}_{1}}\left(\Theta^{\prime}\right)^{-1} \operatorname{Ad}_{\dot{v}_{2}} \Theta^{\prime}$ is an automorphism of $\left(G_{1}^{v_{1}, v_{2}}\right)^{\prime}$.

The connected subgroups of $G_{i}^{v_{1}, v_{2}}$ with Lie algebras $\mathfrak{g}_{i}^{v_{1}, v_{2}} \cap\left(\mathfrak{l}_{i}^{\prime} \oplus \mathfrak{a}_{i}\right)$ and $\mathfrak{g}_{i}^{v_{1}, v_{2}} \cap \operatorname{Ad}_{\dot{v}_{i}}\left(\mathfrak{l}_{i}^{\prime} \oplus \mathfrak{a}_{i}\right)$ will be denoted by $L_{i}^{v_{1}, v_{2}}$ and $\bar{L}_{i}^{v_{1}, v_{2}}$. Note that

$$
\begin{aligned}
& \operatorname{Ad}_{\dot{v}_{2}} \theta: \operatorname{Lie}\left(Z\left(L_{1}^{v_{1}, v_{2}}\right)\right) \rightarrow \operatorname{Lie}\left(Z\left(\bar{L}_{2}^{v_{1}, v_{2}}\right)\right) \text { and } \\
& \theta \operatorname{Ad}_{\dot{v}_{1}}^{-1}: \operatorname{Lie}\left(Z\left(\bar{L}_{1}^{v_{1}, v_{2}}\right)\right) \rightarrow \operatorname{Lie}\left(Z\left(L_{2}^{v_{1}, v_{2}}\right)\right)
\end{aligned}
$$


are linear isomorphisms. For any $\left(v_{1}, v_{2}\right) \in V$ we will denote by $Z_{i}^{v_{1}, v_{2}}$ the following subgroups of the abelian group $Z\left(G_{1}^{v_{1}, v_{2}}\right)^{\circ} \times Z\left(G_{2}^{v_{1}, v_{2}}\right)^{\circ}$ :

$$
\begin{aligned}
& Z_{1}^{v_{1}, v_{2}}=\left\{(x, y) \in Z\left(L_{1}^{v_{1}, v_{2}}\right)^{\circ} \times Z\left(\bar{L}_{2}^{v_{1}, v_{2}}\right)^{\circ} \mid \Theta \mathrm{p}_{+}(x)=\mathrm{p}_{-} \operatorname{Ad}_{\dot{v}_{2}}^{-1}(y)\right\}^{\circ}, \\
& Z_{2}^{v_{1}, v_{2}}=\left\{(x, y) \in Z\left(\bar{L}_{1}^{v_{1}, v_{2}}\right)^{\circ} \times Z\left(L_{2}^{v_{1}, v_{2}}\right)^{\circ} \mid \mathrm{p}_{+} \operatorname{Ad}_{\dot{v}_{1}}^{-1}(x)=\Theta^{-1} \mathrm{p}_{-}(y)\right\}^{\circ} .
\end{aligned}
$$

Define also the following subgroup of $Z\left(G_{1}^{v_{1}, v_{2}}\right)^{\circ} \times Z\left(G_{2}^{v_{1}, v_{2}}\right)^{\circ}$

$$
Z^{v_{1}, v_{2}}=Z_{1}^{v_{1}, v_{2}} Z_{2}^{v_{1}, v_{2}}\left(H_{1}^{\text {ort }} \operatorname{Ad}_{\dot{v}_{1}}\left(H_{1}^{\text {ort }}\right) \times H_{2}^{\text {ort }} \operatorname{Ad}_{\dot{v}_{2}}\left(H_{2}^{\text {ort }}\right)\right) .
$$

Theorem 5.1 The $G^{r}$ double cosets of $G \times G$ are classified by pairs $\left(v_{1}, v_{2}\right)$ of minimal length representatives in $W$ of cosets from $W / W_{i}$, an orbit of the twisted conjugation action $T C^{\dot{v}_{1}, \dot{v}_{2}}$ of $\left(G_{1}^{v_{1}, v_{2}}\right)^{\prime}$ on itself defined by

$$
T C_{g}^{\dot{v}_{1}, \dot{v}_{2}}(f)=g^{-1} f \operatorname{Ad}_{\dot{v}_{1}}\left(\Theta^{\prime}\right)^{-1} \operatorname{Ad}_{\dot{v}_{2}} \Theta^{\prime}(g) ; g, f \in\left(G_{1}^{v_{1}, v_{2}}\right)^{\prime},
$$

and an element of the abelian factor group

$$
\left(Z\left(G_{1}^{v_{1}, v_{2}}\right)^{\circ} \times Z\left(G_{2}^{v_{1}, v_{2}}\right)^{\circ}\right) / Z^{v_{1}, v_{2}} .
$$

This data has to be taken modulo the action of the finite group

$$
\left(\left(G_{1}^{v_{1}, v_{2}}\right)^{\prime} \cap Z\left(G_{1}^{v_{1}, v_{2}}\right)^{\circ}\right) \times\left(\left(G_{1}^{v_{1}, v_{2}}\right)^{\prime} \cap Z\left(G_{2}^{v_{1}, v_{2}}\right)^{\circ}\right)
$$

on $\left(G_{1}^{v_{1}, v_{2}}\right)^{\prime} \times Z\left(G_{1}^{v_{1}, v_{2}}\right)^{\circ} \times Z\left(G_{2}^{v_{1}, v_{2}}\right)^{\circ}$ where an element $\left(q_{1}, q_{2}\right)$ of (5.8) acts on the direct product by multiplication by $\left(q_{1}^{-1} \operatorname{Ad}_{\dot{v}_{1}}\left(\Theta^{\prime}\right)^{-1}\left(q_{2}\right), q_{1}, q_{2}\right)$. (The choice of representatives of $v_{i}$ in the normalizer of the maximal torus $H$ of $G$ is not essential.)

An inductive procedure: Let $\left[\left(l_{1} \dot{w}_{1}, \dot{w}_{2}^{-1} l_{2}\right)\right]$ be an arbitrary $G^{r}$ double coset of $G \times G$, where $l_{i} \in L_{i}$ and $w_{i}$ are minimal length representatives of cosets from $W_{i} \backslash W / W_{i}, i=1,2$.

We define two sequences of reductive Lie subalgebras $\mathfrak{g}_{i}^{k}$ of $\mathfrak{g}$, elements $g_{i}^{k} \in \mathfrak{g}_{i}^{k}$, and $w_{i}^{k} \in W(k \geq 1, i=1,2)$. Set $\mathfrak{g}_{i}^{1}=\mathfrak{l}_{i}, g_{i}^{1}=l_{i}, w_{i}^{1}=w_{i}$. For convenience we also define $\mathfrak{g}_{i}^{0}=\mathfrak{g}$ and extend $\theta$ to a linear isomorphism between $\mathfrak{l}_{1}$ and $\mathfrak{l}_{2}$.

As in the proof of Theorem 4.5 the defined objects will have the following two properties:

(1) $\mathfrak{g}_{1}^{k}$ and

$$
\overline{\mathfrak{g}}_{1}^{k}:=\operatorname{Ad}_{\left(\dot{w}_{1}^{k-1} \ldots \dot{w}_{1}^{1}\right)} \theta^{-1}\left(\mathfrak{g}_{2}^{k}\right)
$$

are reductive subalgebras of $\mathfrak{g}_{1}^{k-1} \subset \mathfrak{g}$ generated by the Cartan subalgebra $\mathfrak{h}$ and by root spaces of simple roots of $\mathfrak{g}$. The same property have the subalgebras $\mathfrak{g}_{2}^{k}$ and

$$
\overline{\mathfrak{g}}_{2}^{k}=\operatorname{Ad}_{\left(\dot{w}_{2}^{k-1} \ldots \dot{w}_{2}^{1}\right)} \theta\left(\mathfrak{g}_{1}^{k}\right)
$$

of $\mathfrak{g}_{2}^{k-1}$.

Let $G_{i}^{k}, \bar{G}_{i}^{k}$ be the connected subgroups of $G$ with Lie algebras $\mathfrak{g}_{i}^{k}, \overline{\mathfrak{g}}_{i}^{k}$ and $W_{i}^{k}, \bar{W}_{i}^{k}$ be their Weyl groups. 
(2) $w_{i}^{k}$ are representatives of minimal length in $W_{i}^{k-1}$ of cosets from $W_{i}^{k} \backslash W_{i}^{k-1} / \bar{W}_{i}^{k}$. If $\mathfrak{g}_{1}^{k+1} \neq \mathfrak{g}_{1}^{k}$ or $\mathfrak{g}_{2}^{k+1} \neq \mathfrak{g}_{2}^{k}$ we define $\mathfrak{g}_{1}^{k+1}$ and $\mathfrak{g}_{2}^{k+1}$ by

$$
\begin{aligned}
& \mathfrak{g}_{1}^{k+1}:=\mathfrak{g}_{1}^{k} \cap \theta^{-1} \operatorname{Ad}_{\left(\dot{w}_{2}^{k} \ldots \dot{w}_{2}^{1}\right)}^{-1}\left(\mathfrak{g}_{2}^{k}\right)=\theta^{-1} \operatorname{Ad}_{\left(\dot{w}_{2}^{k-1} \ldots \dot{w}_{2}^{1}\right)}^{-1}\left(\overline{\mathfrak{g}}_{2}^{k} \cap \operatorname{Ad}_{\dot{w}_{2}^{k}}^{-1}\left(\mathfrak{g}_{2}^{k}\right)\right), \\
& \mathfrak{g}_{2}^{k+1}:=\mathfrak{g}_{2}^{k} \cap \theta \operatorname{Ad}_{\left(\dot{w}_{1}^{k} \ldots \dot{w}_{1}^{1}\right)}^{-1}\left(\mathfrak{g}_{1}^{k}\right)=\theta \operatorname{Ad}_{\left(\dot{w}_{1}^{k-1} \ldots \dot{w}_{1}^{1}\right)}^{-1}\left(\overline{\mathfrak{g}}_{1}^{k} \cap \operatorname{Ad}_{\dot{w}_{1}^{k}}^{-1}\left(\mathfrak{g}_{1}^{k}\right)\right) .
\end{aligned}
$$

Denote with $N_{i \pm}^{k+1}$ and $\bar{N}_{i \pm}^{k+1}$ the unipotent subgroups of $G_{i}^{k}$ generated by one parameter subgroups of positive / negative roots of $\mathfrak{g}_{i}^{k}$ that are not roots of $\mathfrak{g}_{i}^{k+1}$ and $\overline{\mathfrak{g}}_{i}^{k+1}$. Set $\epsilon_{k}=(-1)^{k}$. Let us apply the Bruhat Lemma to $g_{1}^{k} \in G_{1}^{k}, g_{2}^{k} \in G_{2}^{k}$ and the following pairs of parabolic subgroups: $N_{1 \epsilon_{k+1}^{k+1}} \rtimes G_{1}^{k+1}, G_{1}^{k+1} \ltimes \bar{N}_{1+}^{k+1}$ of $G_{1}^{k}$ and $\bar{N}_{2-}^{k+1} \rtimes G_{2}^{k+1}, G_{2}^{k+1} \ltimes N_{2 \epsilon_{k}}^{k+1}$ of $G_{2}^{k}$. We obtain

$$
\begin{aligned}
& g_{1}^{k}=n_{1}^{\prime} g_{1}^{\prime} \dot{w}_{1}^{k+1} g_{1}^{\prime \prime} n_{1}^{\prime \prime}, \\
& g_{2}^{k}=n_{2}^{\prime} g_{2}^{\prime}\left(\dot{w}_{2}^{k+1}\right)^{-1} g_{2}^{\prime \prime} n_{2}^{\prime \prime}
\end{aligned}
$$

for some $g_{1}^{\prime} \in G_{1}^{k+1}, g_{1}^{\prime \prime} \in\left(\bar{G}_{1}^{k+1}\right)^{\prime} g_{2}^{\prime} \in\left(\bar{G}_{2}^{k+1}\right)^{\prime}, g_{2}^{\prime \prime} \in G_{2}^{k+1}, n_{1}^{\prime} \in N_{1 \epsilon_{k+1}}^{k+1}, n_{1}^{\prime \prime \prime} \in \bar{N}_{1+}^{k+1}$, $n_{1}^{\prime \prime} \in \bar{N}_{2-}^{k+1}$, and $n_{2}^{\prime \prime} \in N_{2 \epsilon_{k}}^{k+1}$. The elements $w_{i}^{k+1}$ are minimal length representatives in $W_{i}^{k}$ of cosets from $\bar{W}_{i}^{k+1} \backslash W / W_{i}^{k+1}$. Eqs. (5.11), (5.12) define them uniquely. The elements $g_{i}^{k+1} \in G_{i}^{k+1}$ are defined by

$$
\begin{aligned}
& g_{1}^{k+1}=\left(\left(\Theta^{\prime}\right)^{-1} \operatorname{Ad}_{\left(\dot{w}_{2}^{k} \ldots \dot{w}_{2}^{1}\right)}^{-1} g_{2}^{\prime}\right) g_{1}^{\prime}, \\
& g_{2}^{k+1}=g_{2}^{\prime \prime}\left(\Theta^{\prime} \operatorname{Ad}_{\left(\dot{w}_{1}^{k} \ldots \dot{w}_{1}^{1}\right)}^{-1} g_{1}^{\prime \prime}\right)
\end{aligned}
$$

(see (5.1) and (4.1)). The described procedure has finitely many steps $k=1, \ldots, K$. Denote $v_{i}=w_{i}^{K} \ldots w_{i}^{1}, i=1,2$. For their representatives in $N(H)$ we set $\dot{v}_{i}=\dot{w}_{i}^{K} \ldots \dot{w}_{i}^{1}$. Similarly to the proof of Lemma 4.6 one shows that

$$
\left(v_{1}, v_{2}\right) \in V
$$

and that any pair $\left(v_{1}^{0}, v_{2}^{0}\right) \in V$ can be obtained through the described procedure. At the last step of the procedure

$$
\mathfrak{g}_{i}^{K}=\mathfrak{g}_{i}^{v_{1}, v_{2}}
$$

We will also need the connected subgroups of $G_{i}^{k}$ and $\bar{G}_{i}^{k}$ with Lie algebras $\mathfrak{g}_{i}^{k} \cap\left(\mathfrak{l}_{i}^{\prime} \oplus \mathfrak{a}_{i}\right)$ and $\overline{\mathfrak{g}}_{i}^{k} \cap \operatorname{Ad}_{\left(\dot{w}_{i}^{k-1} \ldots \dot{w}_{i}^{1}\right)}\left(\mathfrak{l}_{i}^{\prime} \oplus \mathfrak{a}_{i}\right)$. They will be denoted by $L_{i}^{k}$ and $\bar{L}_{i}^{k}$. Let $G_{1}^{k, r}$ and $G_{2}^{k, r}$ denote the subgroups of $G_{1}^{k-1} \times G_{2}^{k-1}$ defined by

$$
\begin{aligned}
G_{1}^{k, r}=\quad & \left\{(x, y) \in L_{1}^{k} \times \bar{L}_{2}^{k} \mid \Theta \mathrm{p}_{+}(x)=\mathrm{p}_{-} \operatorname{Ad}_{\left(\dot{w}_{2}^{k-1} \ldots \dot{w}_{2}^{1}\right)}^{-1}(y)\right\}^{\circ} \times \\
& \left(H_{1}^{\text {ort }} N_{1 \epsilon_{k-1}} \times \operatorname{Ad}_{\left(\dot{w}_{2}^{k-1} \ldots \dot{w}_{2}^{1}\right)}\left(H_{2}^{\text {ort }}\right) \bar{N}_{2-}^{k}\right)
\end{aligned}
$$

and

$$
\begin{aligned}
G_{2}^{k, r}=\quad & \left\{(x, y) \in \bar{L}_{1}^{k} \times L_{2}^{k} \mid \mathrm{p}_{+} \operatorname{Ad}_{\left(\dot{w}_{1}^{k-1} \ldots \dot{w}_{1}^{1}\right)}^{-1}(x)=\Theta^{-1} \mathrm{p}_{-}(y)\right\}^{\circ} \times \\
& \left(\operatorname{Ad}_{\left(\dot{w}_{2}^{k-1} \ldots \dot{w}_{2}^{1}\right)}\left(H_{1}^{\text {ort }}\right) \bar{N}_{1+}^{k} \times H_{2}^{\text {ort }} N_{1 \epsilon_{k}}\right) .
\end{aligned}
$$


Similarly to the proof of $(3.20)$, using the fact $\Lambda_{i} \subset H$, one shows that the groups $G_{i}^{k, r}$ are connected. They will play the role of $G^{r}$ (as left and right factor) at the $k$-th step of the procedure. In particular $G_{i}^{1, r}=G^{r}, i=1,2$.

Proof of Theorem 5.1. As in the proof of Theorem 4.5 we construct inductively a sequence of dense, open subsets of the double coset $\left[\left(l_{1} \dot{w}_{1},\left(\dot{w}_{2}\right)^{-1} l_{2}\right)\right]$. The set from the first step consists of the following elements of $G \times G$

$$
\begin{aligned}
& \left(m_{+}\left(\left(\Theta^{\prime}\right)^{-1} l_{+}^{\prime \prime}\right)\left(\mathrm{p}_{+}^{-1} \Theta^{-1} \mathrm{p}_{-}\left(l_{2}^{\prime \prime}\right)\right)\left(\left(\Theta^{\prime}\right)^{-1} l_{-}^{\prime \prime}\right) l_{1} \dot{w}_{1} l_{+}^{\prime} n_{+}^{\prime} l_{*}^{\prime} m_{*}^{\prime} l_{-}^{\prime} n_{-}^{\prime},\right. \\
& \left.n_{+}^{\prime \prime} l_{+}^{\prime \prime} m_{*}^{\prime \prime} l_{*}^{\prime \prime} n_{+}^{\prime \prime} l_{+}^{\prime \prime}\left(\dot{w}_{2}\right)^{-1} l_{2}\left(\Theta^{\prime} l_{+}^{\prime}\right)\left(\mathrm{p}_{-}^{-1} \Theta \mathrm{p}_{+}\left(l_{*}^{\prime}\right)\right)\left(\Theta^{\prime} l_{-}^{\prime}\right) m_{-}\right)
\end{aligned}
$$

where the elements $l_{+}^{\prime}, n_{+}^{\prime}, l_{*}^{\prime}, m_{*}^{\prime}, l_{-}^{\prime}, n_{-}^{\prime}$ belong to the factors of the following dense, open subset of $G_{+}$:

$$
\begin{aligned}
& \left(L_{1}^{1} \cap \operatorname{Ad}_{\dot{w}_{1}^{1}}^{-1}\left(N_{1+}^{1}\right)\right)\left(N_{1+}^{1} \cap \operatorname{Ad}_{\dot{w}_{1}^{1}}^{-1}\left(N_{1+}^{1}\right)\right)\left(L_{1}^{1} \cap \operatorname{Ad}_{\dot{w}_{1}^{1}}^{-1}\left(G_{1}^{1}\right)\right) \times \\
& \times\left(M_{+} \cap \operatorname{Ad}_{\dot{w}_{1}^{1}}^{-1}\left(G_{1}^{1}\right)\right)\left(L_{1}^{1} \cap \operatorname{Ad}_{\dot{w}_{1}^{1}}^{-1}\left(N_{1-}^{1}\right)\right)\left(N_{1+}^{1} \cap \operatorname{Ad}_{\dot{w}_{1}^{1}}^{-1}\left(N_{1-}^{1}\right)\right) .
\end{aligned}
$$

Similarly $n_{+}^{\prime \prime}, l_{+}^{\prime \prime}, m_{*}^{\prime \prime}, l_{*}^{\prime \prime}, n_{+}^{\prime \prime}, l_{+}^{\prime \prime}$, belong to the factors of the following dense, open subset of $G_{-}$

$$
\begin{aligned}
& \left(\bar{N}_{2-}^{1} \cap \operatorname{Ad}_{\dot{w}_{2}^{1}}^{-1}\left(N_{2+}^{1}\right)\right)\left(L_{2}^{1} \cap \operatorname{Ad}_{\dot{w}_{2}^{1}}^{-1}\left(N_{2+}^{1}\right)\right)\left(M_{-} \cap \operatorname{Ad}_{\dot{w}_{2}^{1}}^{-1}\left(G_{2}^{1}\right)\right) \times \\
& \times\left(L_{2}^{1} \cap \operatorname{Ad}_{\dot{w}_{2}^{1}}^{-1}\left(G_{2}^{1}\right)\right)\left(\bar{N}_{2-}^{1} \cap \operatorname{Ad}_{\dot{w}_{2}^{1}}^{-1}\left(N_{2-}^{1}\right)\right)\left(L_{2}^{1} \cap \operatorname{Ad}_{\dot{w}_{2}^{1}}^{-1}\left(N_{2-}^{1}\right)\right) .
\end{aligned}
$$

The two sets (5.18) and $(5.19)$ are constructed according to how $\operatorname{Ad}_{\dot{w}_{1}^{1}}\left(G_{+}\right)$intersects the dense, open subset $N_{1+}^{1} G_{1}^{1} N_{1-}^{1}$ of $G$ and similarly how $\operatorname{Ad}_{\dot{w}_{2}^{1}}\left(G_{-}\right)$intersects $N_{2+}^{1} G_{2}^{1} N_{2-}$. The set (5.17) is obtained by replacing the first component of the right factor $G^{r}$ with (5.18) and the second component of the left factor $G^{r}$ with the set (5.19).

Similarly to (4.23) one shows that the set of elements (5.17) can be represented as

$$
\begin{aligned}
& \left(e,\left(\dot{w}_{2}^{1}\right)^{-1}\right)\left(N_{1+}^{1} \times\left(\operatorname{Ad}_{\dot{w}_{2}^{1}}^{1}\left(\bar{N}_{2-}^{1}\right) \cap N_{2+}^{1}\right)\right)\left(\left(\left(\Theta^{\prime}\right)^{-1} \operatorname{Ad}_{\dot{w}_{2}^{1}}^{-1}, \mathrm{id}\right)\left(\operatorname{Ad}_{\dot{w}_{2}^{1}}\left(\bar{L}_{1}^{2}\right) \cap N_{2+}^{1}\right)\right) \times \\
& \times G_{1}^{2, r}\left(g_{1}^{1}, g_{2}^{1}\right) G_{2}^{2, r} \times \\
& \times\left(\left(\mathrm{id}, \Theta^{\prime} \operatorname{Ad}_{\dot{w}_{1}^{1}}^{-1}\right)\left(\operatorname{Ad}_{\dot{w}_{1}^{1}} \bar{L}_{1}^{1} \cap N_{1-}^{1}\right)\right)\left(\left(\operatorname{Ad}_{\dot{w}_{1}^{1}}\left(\bar{N}_{1+}^{1}\right) \cap N_{1-}^{1}\right) \times N_{2-}^{1}\right)\left(\dot{w}_{1}^{1}, e\right)
\end{aligned}
$$

(recall eqs. (5.15), (5.16) for the definitions of $\left.G_{i}^{2, r}\right)$. One proceeds with the double left $G_{1}^{2, r}$ and right $G_{1}^{2, r}$ coset in $G_{1}^{1} \times G_{2}^{1}$ in the same way as in the proof of Theorem 4.5. Unfortunately the unipotent radicals of the projections of $G_{1}^{k, r}$ and $G_{2}^{k, r}$ on the first component (and also on the second) correspond to roots of different sign for even $k$ and to roots of the same sign for odd $k$. (Compare $N_{1 \epsilon_{k+1}}^{k}, \bar{N}_{1+}^{k}$ and $\bar{N}_{2-}^{k}, N_{2 \epsilon_{k}}^{k}$.) This does not allow obtaining a closed formula for the dimensions of the cosets. The necessary modifications will be made in the next Subsection.

At the last step of the procedure we get the double coset

$$
G_{1}^{K+1, r}\left(g_{1}^{K}, g_{2}^{K}\right) G_{2}^{K+1, r}
$$


in $G_{1}^{v_{1}, v_{2}} \times G_{2}^{v_{1}, v_{2}}$. The groups $N_{i \pm}^{K+1}$ and $\bar{N}_{i \pm}^{K+1}$ are trivial and

$$
\begin{aligned}
& G_{1}^{K+1, r}=\left(H_{1}^{\text {ort }} \times \operatorname{Ad}_{\dot{v}_{2}}\left(H_{2}^{\text {ort }}\right)\right)\left(\left(\mathrm{id}, \operatorname{Ad}_{\dot{v}_{2}} \Theta^{\prime}\right)\left(G_{1}^{v_{1}, v_{2}}\right)^{\prime}\right) Z_{1}^{v_{1}, v_{2}}, \\
& G_{2}^{K+1, r}=\left(\operatorname{Ad}_{\dot{v}_{1}}\left(H_{1}^{\text {ort }}\right) \times H_{2}^{\text {ort }}\right)\left(\left(\operatorname{Ad}_{\dot{v}_{1}}\left(\Theta^{\prime}\right)^{-1}, \mathrm{id}\right)\left(G_{2}^{v_{1}, v_{2}}\right)^{\prime}\right) Z_{2}^{v_{1}, v_{2}} .
\end{aligned}
$$

This is a consequence from

$$
\mathrm{p}_{ \pm}\left(Z\left(L_{i}^{v_{1}, v_{2}}\right)^{\circ}\right)=Z\left(L_{i}^{v_{1}, v_{2}} / \Lambda_{i}\right)^{\circ}
$$

which can be proved as follows. The group on the left is a connected subgroup of the one on the right and the two have one and the same tangent Lie algebras, so they coincide.

Decompose $g_{i}^{K} \in G_{i}^{v_{1}, v_{2}}$ as

$$
g_{i}^{K}=f_{i}^{K} c_{i}^{K}, f_{i}^{K} \in\left(G_{i}^{v_{1}, v_{2}}\right)^{\prime}, c_{i}^{K} \in Z\left(G_{i}^{v_{1}, v_{2}}\right)^{\circ} .
$$

Each pair $\left(f_{i}^{K}, c_{i}^{K}\right)$ is only defined modulo multiplication of $f_{i}^{K}$ and division of $c_{i}^{K}$ by an element from the finite abelian group $\left(G_{i}^{v_{1}, v_{2}}\right)^{\prime} \cap Z\left(G_{i}^{v_{1}, v_{2}}\right)^{\circ}, i=1,2$. Using eqs. (5.22) and (5.23) one rewrites (5.21) as

$$
\left(T C_{\left(G^{\left.v_{1}, v_{2}\right)^{\prime}}\right.}^{\dot{v}_{1}, \dot{v}_{2}}\left(f_{1}^{K} \operatorname{Ad}_{\dot{v}_{1}}\left(\Theta^{\prime}\right)^{-1}\left(f_{2}^{K}\right)^{-1}\right), e\right)\left(\left(c_{1}^{K}, c_{2}^{K}\right) Z^{v_{1}, v_{2}}\right)\left(\left(\mathrm{id}, \Theta^{\prime} \operatorname{Ad}_{\dot{v}_{1}}^{-1}\right)\left(G_{1}^{v_{1}, v_{2}}\right)^{\prime}\right)
$$

(recall the definition (5.5) of the group $Z^{v_{1}, v_{2}}$ ).

The final result of the procedure is

$$
\left[\left(l_{1} \dot{w}_{1}, \dot{w}_{2}^{-1} l_{2}\right)\right]=\left[\left(f_{1}^{K}\left(\operatorname{Ad}_{\dot{v}_{1}}\left(\Theta^{\prime}\right)^{-1}\left(f_{2}^{K}\right)^{-1}\right) c_{1}^{K} \dot{v}_{1}, \dot{v}_{2}^{-1} c_{2}^{K}\right)\right]
$$

and that this coset has the following dense, open subset

$$
\begin{aligned}
& \left(e, \dot{v}_{2}^{-1}\right)\left(\prod_{k=1}^{K} E^{k}\right) \times \\
& \times\left(T C_{\left(G_{1}^{v_{1}, v_{2}}\right)^{\prime}}^{\dot{v}_{1}, f_{1}}\left(f_{1}^{K} \operatorname{Ad}_{\dot{v}_{1}}\left(\Theta^{\prime}\right)^{-1}\left(f_{2}^{K}\right)^{-1}\right), e\right)\left(\left(c_{1}^{K}, c_{2}^{K}\right) Z^{v_{1}, v_{2}}\right)\left(\left(\mathrm{id}, \Theta^{\prime} \operatorname{Ad}_{\dot{v}_{1}}^{-1}\right)\left(G_{1}^{v_{1}, v_{2}}\right)^{\prime}\right) \times \\
& \times\left(\prod_{k=K}^{1} F^{k}\right)\left(\dot{v}_{1}, e\right)
\end{aligned}
$$

where the subsets $E^{k}$ and $F^{k}$ of $G \times G$ are defined by

$$
\begin{aligned}
E^{k}= & \left(N_{1 \epsilon_{k+1}}^{k} \times \operatorname{Ad}_{\left(\dot{w}_{2}^{K} \ldots \dot{w}_{2}^{k+1}\right)}\left(\operatorname{Ad}_{\dot{w}_{2}^{k}}\left(N_{2-}^{k}\right) \cap N_{2 \epsilon_{k}}\right)\right) \times \\
& \times\left(\left(\left(\Theta^{\prime}\right)^{-1} \operatorname{Ad}_{\dot{w}_{2}^{k}}^{-1}, \operatorname{Ad}_{\left(\dot{w}_{2}^{K} \ldots \dot{w}_{2}^{k+1}\right)}\right)\left(\operatorname{Ad}_{\dot{w}_{2}^{k}}\left(\bar{L}_{2}^{k}\right) \cap N_{2 \epsilon_{k+1}}^{k}\right)\right),
\end{aligned}
$$

and

$$
\begin{aligned}
F^{k}= & \left(\left(\operatorname{Ad}_{\left(\dot{w}_{1}^{K} \ldots \dot{w}_{1}^{k+1}\right)}, \Theta^{\prime} \operatorname{Ad}_{\dot{w}_{1}^{k}}^{-1}\right)\left(\operatorname{Ad}_{\dot{w}_{1}^{k}}\left(\bar{L}_{1}^{k}\right) \cap\left(N_{1 \epsilon_{k}}^{k}\right)\right)\right) \times \\
& \times\left(\operatorname{Ad}_{\left(\dot{w}_{1}^{K} \ldots \dot{w}_{1}^{k+1}\right)}\left(\operatorname{Ad}_{\dot{w}_{1}^{k}}\left(\bar{N}_{1+} \cap N_{1 \epsilon_{k}}^{k}\right) \times N_{2, \epsilon_{k}}^{k}\right)\right) .
\end{aligned}
$$


(Here as before $\epsilon_{k}=(-1)^{k}$.)

To the coset (5.25) one associates the pair $\left(v_{1}, v_{2}\right) \in V$, the $T C_{\left(G^{v_{1}, v_{2}}\right)^{\prime}}^{\dot{v}_{1} \dot{v}_{2}}$ orbit of $f_{1}^{K} v_{1}\left(\Theta^{\prime}\right)^{-1}\left(f_{2}^{K}\right) \in G^{v_{1}, v_{2}}$, and the coset

$$
\left(c_{1}^{K}, c_{2}^{K}\right) Z^{v_{1}, v_{2}} \in\left(Z\left(G_{1}^{v_{1}, v_{2}}\right)^{\circ} \times Z\left(G_{2}^{v_{1}, v_{2}}\right)^{\circ}\right) / Z^{v_{1}, v_{2}} .
$$

This is only defined modulo the action of the finite group (5.7) due to the freedom in the definition of $f_{i}^{K}$ and $c_{i}^{K}$.

Analogously to the proof of Theorem 4.5 one shows that two sets of the type (5.26) intersect if and only if they correspond to one and the same pair $\left(v_{1}, v_{2}\right) \in V$, orbit of $T C^{\dot{v}_{1}, \dot{v}_{2}}$, and cosets of the abelian factor group (5.7), modulo the action of the group (5.8). The equivalence relation respects the nonuniqueness in the definition of $c_{i}^{K}$ and $f_{i}^{K}$.

From this proof one also derives:

Corollary 5.2 If the Poisson structure on the group $G$ comes from an $r$-matrix for which $\mathfrak{g}_{ \pm} \supset \mathfrak{h}$ and the groups $\Lambda_{i}$ are trivial then the $G^{r}$ double cosets of $G \times G$ are classified by a pair of minimal representatives $\left(v_{1}, v_{2}\right)$ in $W$ of cosets from $W / W_{i}$ and an orbit of the twisted conjugation action of $G_{1}^{v_{1}, v_{2}}$ on itself defined by

$$
\widetilde{T C}_{g}^{\dot{v}_{1}, \dot{v}_{2}}(f)=g^{-1} f\left(\operatorname{Ad}_{\dot{v}_{1}} \Theta^{-1} \operatorname{Ad}_{\dot{v}_{2}} \Theta(g)\right) ; f, g \in G_{1}^{v_{1}, v_{2}}
$$

(Simply connectedness for $G^{\prime}$ is not assumed.)

\subsubsection{Another view on $G^{r}$ double cosets and a dimension formula}

The goal of this Subsection is to prove a formula for the dimensions of the $G^{r}$ double cosets of $G \times G$. The idea is to conjugate the group $G^{r}$ by some elements of $N(H) \times N(H)$ in such a way that the iterative procedure of the proof of Theorem 5.1 is still applicable to the cosets of the resulting subgroups of $G \times G$. In the same time the corresponding groups $G_{1}^{k, r}$ and $G_{2}^{k, r}$ from the iteration will have unipotent radicals of their first and second components, of opposite sign (independent of the parity of $k$ ).

Let $w^{\max }$ and $w_{i}^{\max }$ be the elements of maximal length in the Weyl groups $W$ and $W_{i}, i=1,2$. Denote with $u_{i}^{\max }$ the representatives of minimal length in $W$ of the cosets $w^{\max } W_{i} \in W / W_{i}$. More explicitly:

$$
u_{i}^{\max }=w^{\max }\left(w_{i}^{\max }\right)^{-1} .
$$

Denote

$$
\begin{aligned}
\widehat{G}_{1}^{r} & =\left(\mathrm{id} \times \operatorname{Ad}_{\dot{u}_{2}^{\max }}\right) G^{r} \subset G \times G, \\
\widehat{G}_{2}^{r} & =\left(\operatorname{Ad}_{\dot{u}_{1}^{\max }} \times \mathrm{id}\right) G^{r} \subset G \times G .
\end{aligned}
$$

The double - left $\widehat{G}_{1}^{r}$ and right $\widehat{G}_{2}^{r}$ cosets of $G \times G$ will be called for simplicity $\widehat{G}_{1}^{r}-\widehat{G}_{2}^{r}$ cosets of $G \times G$. There is a simple connection between them and the $G^{r}$ double cosets of $G \times G$ :

$$
\left[\left(g_{1}, g_{2}\right)\right]=\left(e,\left(\dot{u}_{2}^{\max }\right)^{-1}\right)\left(\widehat{G}_{1}^{r}\left(g_{1}\left(\dot{u}_{1}^{\max }\right)^{-1}, \dot{u}_{2}^{\max } g_{2}\right) \widehat{G}_{2}^{r}\right)\left(\dot{u}_{1}^{\max }, e\right) .
$$


Theorem 5.3 1. Any $\widehat{G}_{1}^{r}-\widehat{G}_{2}^{r}$ coset of $G \times G$ is of the form

$$
\widehat{G}_{1}^{r}\left(f c_{1} \dot{v}_{1}\left(\dot{u}_{1}^{\max }\right)^{-1},\left(\dot{v}_{2}\left(\dot{u}_{2}^{\max }\right)^{-1}\right)^{-1} c_{2}\right) \widehat{G}_{2}^{r}
$$

where $v_{i}$ are minimal length representatives in $W$ of cosets from $W / W_{i}, f \in\left(G_{1}^{v_{1}, v_{2}}\right)^{\prime}$, and $c_{i} \in Z\left(G_{i}^{v_{1}, v_{2}}\right)^{\circ}$. All such cosets are classified by the same data as in Theorem 5.1 and are related to the $G^{r}$ double cosets of $G \times G$ via

$$
\left[\left(f c_{1} \dot{v}_{1}, \dot{v}_{2}^{-1} c_{2}\right)\right]=\left(e,\left(\dot{u}_{2}^{\max }\right)^{-1}\right)\left(\widehat{G}_{1}^{r}\left(f c_{1} \dot{v}_{1}\left(\dot{u}_{1}^{\max }\right)^{-1}, \dot{u}_{2}^{\max } \dot{v}_{2}^{-1} c_{2}\right) \widehat{G}_{2}^{r}\right)\left(\dot{u}_{1}^{\max }, e\right) .
$$

2. The dimension of the cosets (5.30) and (5.31) is

$$
2 \operatorname{dim} \mathfrak{g}-2 \operatorname{dim} \mathfrak{n}_{+}-\operatorname{dim}\left(\mathfrak{g}_{1}^{v_{1}, v_{2}}\right)^{\prime}+l\left(v_{1}\right)+l\left(v_{2}\right)+\operatorname{dim} T C_{\left(G_{1}^{v_{1}, v_{2}}\right)^{\prime}}^{\dot{v}_{1}, \dot{2}_{2}}(f)-\operatorname{dim} Z^{v_{1}, v_{2}} .
$$

Proof. The images of $\mathfrak{l}_{i}, L_{i}, A_{i}, \mathfrak{m}_{ \pm}$, and $M_{ \pm}$under conjugation with $\dot{u}_{i}^{\max }$ will be denoted by $\widehat{\mathfrak{l}}_{i}, \widehat{L}_{i}, \widehat{A}_{i}, \widehat{\mathfrak{m}}_{\mp}$, and $\widehat{M}_{\mp}$.

Since $w^{\max }, w^{\max }$ are the elements of maximal length in $W, W_{i}$, they map (simple) positive roots of $\mathfrak{g}, \mathfrak{l}_{i}$ into (simple) negative roots of the same algebras. Formula (5.28) for $u_{i}^{\max }$ implies that $\operatorname{Ad}_{\dot{u}_{i}^{\max } \text { maps }}$

(1) (simple) positive roots of $\mathfrak{l}_{i}$ into (simple) positive roots of $\widehat{\mathfrak{l}}_{i}$, and of $\widehat{\mathfrak{l}}_{i}$.

(2) positive roots of $\mathfrak{g}$ that are not roots of $\mathfrak{l}_{i}$ into negative roots of $\mathfrak{g}$ that are not roots

From (1) we get that $\widehat{\mathfrak{l}}_{i}$ are reductive subalgebras of $\mathfrak{g}$ generated by $\mathfrak{h}$ and root spaces of simple roots of $\mathfrak{g}$. Property (2) implies that the Lie algebras $\widehat{\mathfrak{m}}_{ \pm}$are spanned by negative root spaces of $\mathfrak{g}$ and $\left(u_{i}^{\max }\right)^{-1}\left(\mathfrak{h}_{i}^{\text {ort }}\right)$.

The groups $G_{i}^{r}$ can be more explicitly written as

$$
\begin{aligned}
& \widehat{G}_{1}^{r}=\left(\widehat{M}_{-}, M_{+}\right)\left\{(x, y) \in\left(\widehat{L}_{1}^{\prime} \widehat{A}_{1}\right) \times\left(L_{2}^{\prime} A_{2}\right) \mid \Theta \mathrm{p}_{+} \operatorname{Ad}_{\dot{u}_{1}^{\max }}^{-1}(x)=y\right\}, \\
& \widehat{G}_{2}^{r}=\left(M_{-}, \widehat{M}_{+}\right)\left\{(x, y) \in\left(L_{1}^{\prime} A_{1}\right) \times\left(\widehat{L}_{2}^{\prime} \widehat{A}_{2}^{\prime}\right) \mid \Theta \mathrm{p}_{+}(x)=\mathrm{p}_{-} \operatorname{Ad}_{\dot{u}_{2}^{\max }}^{-1}(y)\right\} .
\end{aligned}
$$

The two groups are of the same type as $G^{r}$. On Lie algebra level the role of the Cayley transformation $\theta: \mathfrak{l}_{1}^{\prime} \oplus \mathfrak{a}_{1} \rightarrow \mathfrak{l}_{2}^{\prime} \oplus \mathfrak{a}_{2}$ is played by the map

$$
\theta \operatorname{Ad}_{\dot{u}_{1}^{\max }}^{-1}: \widehat{\mathfrak{l}}_{1}^{\prime} \oplus \widehat{\mathfrak{a}}_{1} \rightarrow \mathfrak{l}_{2}^{\prime} \oplus \mathfrak{a}_{2}
$$

for the first group, and by

$$
\operatorname{Ad}_{\dot{u}_{2}^{\max }} \theta: \mathfrak{l}_{1}^{\prime} \oplus \mathfrak{a}_{1} \rightarrow \widehat{\mathfrak{l}}_{2} \oplus \widehat{\mathfrak{a}}_{2}
$$

for the second one.

The procedure from the proof of Theorem 5.1 can be applied to describe the set of $\widehat{G}_{1}^{r}-\widehat{G}_{2}^{r}$ cosets of $G \times G$. It starts with $\mathfrak{g}_{1}^{1}=\widehat{\mathfrak{l}}_{1}, \overline{\mathfrak{g}}_{1}^{1}=\mathfrak{l}_{1}, \mathfrak{g}_{2}^{1}=\mathfrak{l}_{2}$, and $\overline{\mathfrak{g}}_{2}^{1}=\widehat{\mathfrak{l}}_{2}$. The final result depends on a choice of a pair $\left(\widehat{v}_{1}, \widehat{v}_{2}\right)$ of minimal length representatives in $W$ of cosets from $W / \widehat{W}_{i}$. Denote the set of such pairs by $\widehat{V}$ and recall the definition of the set $V$ from Sect. 5.1.1. Properties (1)-(2) of the elements $u_{i}^{\max } \in W$ imply

$$
\left(v_{1}, v_{2}\right) \in V \text { if and only if }\left(v_{1}\left(u_{1}^{\max }\right)^{-1}, v_{2}\left(u_{2}^{\max }\right)^{-1}\right) \in \widehat{V} \text {. }
$$


For any $\left(v_{1}, v_{2}\right) \in V$ we will use the representatives $\dot{v}_{1}\left(\dot{u}_{1}^{\max }\right)^{-1}, \dot{v}_{2}\left(\dot{u}_{2}^{\max }\right)^{-1}$ of $v_{1}\left(u_{1}^{\max }\right)^{-1}$, $v_{2}\left(u_{2}^{\max }\right)^{-1}$ in $N(H)$. As in Theorem 5.1 the procedure for the $\widehat{G}_{1}^{r}-\widehat{G}_{2}^{r}$ cosets of $G \times G$ gives that any such coset is equal to a coset of the form (5.30) for some pair $\left(v_{1} u_{1}^{\max }, v_{2} u_{2}^{\max }\right) \in \widehat{V}$ (i.e. $\left.\left(v_{1}, v_{2}\right) \in V\right)$.

The important point now is that at each step of the procedure the unipotent radicals of the first and second components of the (new) groups $G_{1}^{k, r}$ and $G_{2}^{k, r}$ have opposite signs. This gives a closed formula for the dimension of the coset (5.30):

$$
2 \operatorname{dim} \mathfrak{g}-\operatorname{dim}\left(\mathfrak{g}_{1}^{v_{1}, v_{2}}\right)^{\prime}-l\left(v_{1}\left(u_{1}^{\max }\right)^{-1}\right)-l\left(v_{2}\left(u_{2}^{\max }\right)^{-1}\right)+\operatorname{dim} T C_{\left(G_{1}^{v_{1}, v_{2}}\right)^{\prime}}^{\dot{v}_{1} \dot{v}_{2}}(f)-\operatorname{dim} Z^{v_{1}, v_{2}} .
$$

(The proof is identical to the one of the dimension formula (4.11).) Formula (5.32) follows from it and

$$
l\left(v_{i} u_{i}^{\max }\right)=\operatorname{dim} \mathfrak{n}_{+}-l\left(v_{i}\right)
$$

The last equation is a consequence from properties (1)-(2) of $u_{i}^{\max }$ and the fact that the length of $w \in W$ is equal to the number of positive roots $\alpha$ of $\mathfrak{g}$ for which $w(\alpha)$ is a negative root.

\subsection{The group $d(G) \cap G^{r}$}

Let $\Sigma$ denote the group $d(G) \cap G^{r}$. It is a discrete subgroup of $G \times G$ since $d(\mathfrak{g}) \cap \mathfrak{g}^{r}=0$.

If $(x, y) \in \Sigma$ then $y=x$ and $\mathrm{p}_{+}(x)=\mathrm{p}_{-}(x)$. So $\left(x, \mathrm{p}_{+}(x)\right) \in \Sigma_{-}$and one can define a map

$$
\phi: \Sigma \rightarrow \Sigma_{-} \text {, by } \phi(x, y)=\left(x, \mathrm{p}_{+}(x)\right)
$$

(recall the notation from Sect. 4.2). It is an isomorphism because of (3.20).

Proposition 5.4 For a Poisson structure on $G$ for which $G^{r}$ is a closed subgroup of $G \times G$ the groups $\Sigma$ and $\Sigma_{-}$are canonically isomorphic via (5.33). For any factorizable Poisson structure on $G$

$$
\Sigma=i(H) \cap G^{r}
$$

In the general case (5.34) is proved in the same way as Theorem 4.12 .

Note that all points of $\Sigma$ are fixed under the dressing action of $G^{r}$ on $G$ (i.e. they are 0 dimensional leaves of $G$ ). Thus (5.34) can be viewed as a dressing analog of the classical fact that the center of a reductive group $R$ belongs to any maximal torus of $R$. The theorem deals with a fixed torus because the Poisson structure depends on the choice of the torus.

\subsection{Symplectic leaves of $\mathrm{G}$}

As a direct consequence from the results of the previous Subsections and Theorems 1.9 and 1.10, we obtain the following classification result for the set of symplectic leaves on the Poisson-Lie group $G$.

Theorem 5.5 Let $G$ be a complex reductive Poisson-Lie group with simply connected derived subgroup $G^{\prime}$. 
1. The symplectic leaves in $d(G) / \Sigma$ are classified by the data of Theorem 5.1. The dimension of the leaf corresponding to the pair of minimal length representatives $\left(v_{1}, v_{2}\right)$ of cosets from $W / W_{i}$ and the $T C^{\dot{v}_{1}, \dot{v}_{2}}$ orbit through $f \in\left(G_{1}^{v_{1}, v_{2}}\right)^{\prime}$ is

$$
\operatorname{dim}\left(\mathfrak{l}_{1}^{\prime} \oplus \mathfrak{a}_{1}\right)+2 \operatorname{dim} \mathfrak{h}_{1}^{\text {ort }}-\operatorname{dim}\left(\mathfrak{g}_{1}^{v_{1}, v_{2}}\right)^{\prime}+l\left(v_{1}\right)+l\left(v_{2}\right)+\operatorname{dim} T C_{\left(G_{1}^{v_{1}, v_{2}}\right)^{\prime}}^{\dot{v}_{1}, \dot{2}_{2}}\left(f_{1}\right)-\operatorname{dim} Z^{v_{1}, v_{2}} .
$$

2. The symplectic leaves of $G$ are coverings of the ones of $d(G) / \Sigma$ under the projection $G \rightarrow d(G) / \Sigma$. The group $\Sigma$ can be computed explicitly from Proposition 5.4.

In a particular (but important) case this Theorem simplifies a lot (see Corollaries 5.2 and 4.13).

Corollary 5.6 Let $G$ be a factorizable complex reductive Poisson-Lie group for which the associated r-matrix satisfies $\mathfrak{g}_{ \pm} \supset \mathfrak{h}$ and the groups $\Lambda_{i}$ are trivial.

Then the symplectic leaves of $d(G) / \Sigma$ are classified by a pair of minimal length representatives $\left(v_{1}, v_{2}\right)$ of cosets from $W / W_{i}$ and an orbit of the twisted conjugation action $\widetilde{T C}^{\dot{v}_{1}, \dot{v}_{2}}$ of $G_{1}^{v_{1}, v_{2}}$ on itself defined in (5.27).

The dimension of the leaf that corresponds to this data is

$$
\operatorname{dim} \mathfrak{l}_{1}-\operatorname{dim} \mathfrak{g}_{1}^{v_{1}, v_{2}}+\operatorname{dim} T C_{G_{1}^{v_{1}, v_{2}}}^{\dot{v}_{1}, \dot{v}_{2}}(f)+l\left(v_{1}\right)+l\left(v_{2}\right) .
$$

The group $\Sigma$ is explicitly given by

$$
\Sigma=\{(h, h) \mid h \in H, \Theta(h)=h\} \cong \operatorname{ker} /(1-\theta)(\text { ker })
$$

where ker denotes the kernel of the exponential map for the maximal torus $H$.

Remark 5.7 Consider the special choice of the data of Theorem 5.5 and Corollary 5.6 when $v_{2}$ is taken the identity element id of $W$ and only a subgroup $F_{1}$ of the abelian factor group $\left(Z\left(G_{1}^{v_{1}, \text { id }}\right)^{\circ} \times Z\left(G_{2}^{v_{1}, \text { id }}\right)^{\circ}\right) / Z^{v_{1} \text {,id }}$ is considered. It is defined as the image of the projection of the subtorus $Z\left(G_{1}^{v_{1} \text {,id }}\right)^{\circ} \times e$ of $Z\left(G_{1}^{v_{1}, \text { id }}\right)^{\circ} \times Z\left(G_{2}^{v_{1}, \text { id }}\right)^{\circ}$. The simplectic leaves of $G$ corresponding to this special choice of the data are the symplectic leaves of the Poisson subgroup $G_{-}$of $G$.

The connection with Theorem 4.16 and Corollary 4.17 is as follows. For any minimal representative $v_{1}$ in $W$ of a coset from $W / W_{1}$ one has $G_{1}^{v_{1} \text {,id }}=G^{v_{1}}$ and the twisted conjugation actions $T C^{\dot{v}_{1}, e}$ and $T C^{\dot{v}_{1}}$ are the same. Using

$$
Z_{1}^{v_{1}, \mathrm{id}}=\left\{(x, y) \in Z\left(L_{1}^{v_{1}, \mathrm{id}}\right)^{\circ} \times Z\left(L_{2}^{v_{1}, \mathrm{id}}\right)^{\circ} \mid \Theta \mathrm{p}_{+}(x)=\mathrm{p}_{-}(y)\right\}^{\circ}
$$

one identifies the part of the data of Theorems 4.5 and 5.1 coming from the abelian factor groups

$$
F_{1} \cong Z\left(G^{v_{1}}\right)^{\circ} /\left(\operatorname{Cong}^{v_{1}}\left(L^{v_{1}}\right) H_{1}^{\text {ort }} \operatorname{Ad}_{\dot{v}_{1}}\left(H_{1}^{\text {ort }}\right)\right) .
$$

The fact that the groups $L_{i}^{\prime}$ are simply connected implies $\Lambda_{i} \in Z\left(L_{i}\right)^{\circ}$. From it one also gets

$$
\Lambda_{1}^{v_{1}}=d\left(\left(G^{v_{1}}\right)^{\prime} \cap Z\left(G^{v_{1}}\right)^{\circ}\right)\left(e, \Lambda_{1}\right) \subset\left(G^{v_{1}}\right)^{\prime} \times Z\left(G^{v_{1}}\right)^{\circ}
$$


where $d($.$) stays for the diagonal embedding. This gives the connection between the$ "modulo" parts of Theorems 4.5 and 5.1.

The iterative procedures from the proofs of these Theorems are also related. Let us start with the $G^{r}$ double coset of the element $\left(l_{1} \dot{w}_{1}, e\right) \in G \times G$ and the $i_{+}\left(G_{+}\right)$double coset of the same element but considered in $G \times\left(\left(L_{1}^{\prime} \times A_{1}\right) / \Lambda_{1}\right)$. The reductive Lie algebras $\mathfrak{g}^{k}, \overline{\mathfrak{g}}^{k}$ and $\mathfrak{g}_{1}^{k}, \overline{\mathfrak{g}}_{1}^{k}$ from these proofs are related by

$$
\begin{aligned}
& \mathfrak{g}_{1}^{2 k}=\mathfrak{g}^{k}, \\
& \overline{\mathfrak{g}}_{1}^{2 k-1}=\overline{\mathfrak{g}}^{k} .
\end{aligned}
$$

At odd (even) steps $k$ the algebras $\mathfrak{g}_{1}^{k}$ (respectively $\overline{\mathfrak{g}}_{1}^{k}$ ) are kept unchanged.

Example 5.8 If the complex semisimple group $G$ is equipped with the standard Poisson structure then any double $G^{r}$ coset of $G \times G$ is equal to a coset of the form $\left[\left(h_{1} \dot{v}_{1}, \dot{v}_{2}^{-1} h_{2}\right)\right]$ for some $h_{i} \in H$ and $v_{i} \in W$. The procedure from the proof of Theorem 5.1 has only one step and it produces the following representation of the above coset (see also Corollary 5.2)

$$
\begin{aligned}
& \left(\dot{v}_{2}^{-1}, e\right)\left(N_{+} \times\left(\operatorname{Ad}_{\dot{v}_{2}}\left(N_{-}\right) \cap N_{+}\right)\right)\left(\widetilde{T C} \dot{v}_{H}^{\dot{v}_{1}} \dot{v}_{2}\left(h_{1} \operatorname{Ad}_{\dot{v}_{1}}\left(h_{2}\right)\right), e\right) \times \\
& \times\left\{\left(h^{-1}, \operatorname{Ad}_{\dot{v}_{1}}(h)\right) \mid h \in H\right\}\left(\left(\operatorname{Ad}_{\dot{v}_{1}}\left(N_{+}\right) \cap N_{-}\right) \times N_{-}\right)\left(\dot{v}_{1}, e\right),
\end{aligned}
$$

where

$$
\widetilde{T C}_{h}^{\dot{v}_{1}, \dot{v}_{2}}\left(h^{\prime}\right)=h^{-1} h^{\prime} \operatorname{Ad}_{\dot{v}_{1} \dot{v}_{2}}(h) .
$$

All such double cosets and thus all symplectic leaves of $d(G) / \Sigma$ are classified by a pair $\left(v_{1}, v_{2}\right) \in W$ and an orbit of the action (5.36). Both Corollary 5.6 and (5.35) imply that the dimension of the corresponding symplectic leaf is

$$
\operatorname{dim} \widetilde{T C}_{H}^{\dot{v}_{1}, \dot{v}_{2}}(h)+l\left(v_{1}\right)+l\left(v_{2}\right)
$$

The group $\Sigma$ is isomorphic to the group $\Sigma_{-}$from Example 4.14 (see (4.53) and (4.54)). This Example agrees with the results in [10, 13].

Example 5.9 Here we discuss the set of symplectic leaves of the group $S L(n+1)$ equipped with Cremmer-Gervais Poisson structure.

The first step is the classification of the $S L(n+1)^{r}$ double cosets of $\mathrm{D}(S L(n+1))$. Recall from Example 4.11 that the minimal length representatives in $W \cong S^{n+1}$ of the cosets from $W / W_{1} \cong S^{n+1} / S_{1}^{n}$ are the permutations $\sigma_{1}^{j}, j=0, \ldots, n$ defined in (4.34). In the same way one finds that the minimal length representatives for the cosets from $W / W_{2} \cong S^{n+1} / S_{2}^{n}$ are the permutations

$$
\sigma_{2}^{k}=(1 \ldots(n+1-k))^{-1}, k=0, \ldots, n .
$$

As in Example 4.11 we will assume that all elements of the Weyl group of $S L(n+1)$ are represented in $N(H)$ by multiples of permutation matrices. 
(1) Case $j+k \geq n$. The stable subgroup $G_{1}^{\dot{\sigma}_{1}^{j}, \dot{\sigma}_{2}^{k}}$ is given by

$G_{1}^{\dot{\sigma}_{1}^{j}, \dot{\sigma}_{2}^{k}}=\left\{\operatorname{diag}\left(a_{1}, \ldots, a_{n-k}, A, a_{j+1}, \ldots, a_{n+1}\right) \mid a_{p} \in \mathbb{C}, A \in G L(j+k-n), \operatorname{det} A \prod_{p} a_{p}=1\right\}$.

Under the twisting conjugation action $T C^{\dot{\sigma}_{1}^{j}, \dot{\sigma}_{2}^{k}}$ of this this group on itself the element $\operatorname{diag}\left(a_{1}, \ldots, a_{n-k}, A, a_{j+1}, \ldots, a_{n+1}\right)$ acts as follows

$$
\begin{aligned}
& \operatorname{diag}\left(b_{1}, \ldots, b_{n-k}, B, b_{j+1}, \ldots, b_{n+1}\right) \mapsto \\
& \operatorname{diag}\left(a_{1}^{-1} b_{1} a_{\mu(1)}, \ldots, a_{n-k}^{-1} b_{n-k} a_{\mu(n-k)}, A^{-1} B A, a_{j+1}^{-1} b_{j+1} a_{\mu(j+1)}, \ldots, a_{n+1}^{-1} b_{n+1} a_{\mu(n+1)}\right)
\end{aligned}
$$

where the permutation $\mu \in S^{n+1}$ is given by

$$
\mu=((j+1) \ldots(n+1)(n-k) \ldots 1) .
$$

There is a one to one correspondence between the set of $T C^{\dot{\sigma}_{1}^{j}, \dot{\sigma}_{2}^{k}}$ orbits on $G_{1}^{\dot{\sigma}_{1}^{j}, \dot{\sigma}_{2}^{k}}$ and the conjugation orbits of $G L(j+k-n)$. To the orbit of the element (5.37) of $G_{1}^{\dot{\sigma}_{1}^{j}, \dot{\sigma}_{2}^{k}}$ is associated the conjugation orbit of $B \in G L(j+k-n)$.

Therefore the $S L(n+1)^{r}$ double cosets of $S L(n+1)^{\times 2} \cong \mathrm{D}(S L(n+1))$ and the symplectic leaves of $S L(n+1) / \Sigma$ corresponding to the permutations $\sigma_{1}^{j}, \sigma_{2}^{k}$ with $j+k \geq n$ are classified by the conjugation orbits of $G L(j+k-n)$ on itself. The dimension of the leaf corresponding of the orbit trough $B \in G L(j+k-n)$ is

$$
(2 n-j-k)(j+k+1)+\operatorname{dim}\left\{\operatorname{Ad}_{A} B \mid A \in G L(j+k-n)\right\} .
$$

The group $\Sigma$ coincides with the group $\Sigma_{-}$from Example 4.11 (see (4.55)) and the symplectic leaves in $S L(n+1)$ are coverings of the ones of $S L(n+1) / \Sigma$ under the covering $\operatorname{map} S L(n+1) \rightarrow S L(n+1) / \Sigma$.

(2) Case $j+k \leq n-1$. Then the stable subgroup $G_{1}^{\dot{\sigma}_{1}^{j}, \dot{\sigma}_{2}^{k}}$ is $\left\{\operatorname{diag}\left(a_{1}, \ldots, a_{j+1}, A, a_{n-k+1}, \ldots, a_{n+1}\right) \mid a_{p} \in \mathbb{C}, A \in G L(n-j-k-1), \operatorname{det} A \prod_{p} a_{p}=1\right\}$.

The $T C^{\dot{\sigma}_{1}^{j}, \dot{\sigma}_{2}^{k}}$ action of the element $\operatorname{diag}\left(a_{1}, \ldots, a_{j+1}, A, a_{n-k+1}, \ldots, a_{n+1}\right)$ on $\operatorname{diag}\left(b_{1}, \ldots, b_{j+1}, B, b_{n-k+1}, \ldots, b_{n+1}\right)$ gives the element

$\operatorname{diag}\left(a_{1}^{-1} b_{1} a_{\mu(1)}, \ldots, a_{j+1}^{-1} b_{j+1} a_{\mu(j+1)}, A^{-1} B A, a_{n-k+1}^{-1} b_{n-k+1} a_{\mu(n-k+1)}, \ldots, a_{n+1}^{-1} b_{n+1} a_{\mu(n+1)}\right)$

where

$$
\mu=(1 \ldots(j+1))^{-1}((n-k+1) \ldots(n+1)) \in S^{n+1} .
$$

The symplectic leaves of $S L(n+1) / \Sigma$ corresponding to $\sigma_{1}^{j}, \sigma_{2}^{k}$ in the case $j+k \leq n-1$ are classified by the conjugation orbits of $G L(n-j-k-1)$ on itself and a complex nonzero number. (It comes from the fact that the product of the entries $a_{1}, \ldots, a_{j+1}$ is invariant under the twisting conjugation action.) The dimension of the leaf corresponding to the orbit through $B \in G L(n-j-k+1)$ is

$$
(2 n-j-k-2)(j+k+1)+2 n+\operatorname{dim}\left\{\operatorname{Ad}_{A} B \mid A \in G L(n-j-k-1)\right\} .
$$

The group $\Sigma$ is the same as in case (1) and the symplectic leaves of $S L(n+1)$ are recovered in the same way. 


\section{References}

[1] Belavin, A. A. and Drinfeld, V. G.: Triangle equations and simple Lie algebras. Math. Phys. Rev. 4, 93-165 (1984)

[2] Chari, V. and Pressley, A.: A guide to quantum groups. Cambridge Univ. Press, 1994

[3] Cremmer, E., and Gervais, J.-L.: The quantum group structure associated to non-linearly extended Virasoro algebras. Commun. Math. Phys. 134, 619-632 (1990)

[4] Dixmier, J.: Enveloping algebras. AMS Graduate Studies in Mathematics, 1996 edition

[5] Duflo, M.: Théorie de Mackay pour les groupes de Lie algébriques. Acta Math. 149, 153-213 (1982)

[6] Etingof, P., Schedler, T., and Schiffmann, O.: Explicit quantization of dynamical $r$-matrices for finite dimensional semisimple Lie algebras. preprint math.QA/9912009 (1999)

[7] Etingof, P. and Shiffmann, O.: Lectures on Quantum groups. International Press, 1998

[8] De Concini, C., Kac, V. G., and Procesi, C.: Some quantum analogs of solvable Lie groups. In: Geometry and analysis. Papers presented at the Bombay colloquium, India, January 6-14, 1992, pp. 41-65.

[9] Hodges, T. J.: On the Cremmer-Gervais quantization of $S L(n)$. IMRN 1995, No. 10, 465-481.

[10] Hodges, T. J. and Levasseur, T.: Primitive ideals of $C_{q}[S L(3)]$. Commun. Math. Phys. 156, 581-605 (1993)

[11] Hodges, T. J. and Levasseur, T.: Primitive ideals of $C_{q}[S L(n)]$. J. Algebra 168, 455-468 (1994); Primitive ideals of $C_{q}[G]$. preprint (1992)

[12] Hodges, T. J., Levasseur, T., and Toro, M.: Algebraic structure of multiparameter quantum groups. Adv. Math. 126, 52-92 (1997)

[13] Hoffmann, T., Kellendonk, J., Kutz, N., and Reshetikhin, N.: Factorizational dynamics and Coxeter-Toda lattices. preprint solv-int/9906013 (1999)

[14] Humphreys, J.: Linear algebraic groups. Graduate Text in Mathematics V. 21. Springer-Verlag, 1975

[15] Humphreys, J.: Reflection groups and Coxeter groups. Cambridge Univ. Press, 1990

[16] Joseph, A.: Primitive ideals in enveloping algebras. ICM Proceedings. Warsaw 1983 
[17] Joseph, A.: Idéaux premiers et primitifs de l'algèrbre des fonctions sur un groupe quantique. C. R. Acad. Sci. Paris 316 I, 1139-1142 (1993); On the prime and primitive spectrum of the algebra of functions on a quantum group. J. Algebra 169, 441-511 (1994)

[18] Joseph, A.: Quantum groups and their primitive ideals. Springer Verlag, 1995

[19] Korogodski, L. I. and Soibelman, Y. S.: Algebras of functions on quantum groups: Part I. AMS Math. Surveys and Monographs V. 56, 1998

[20] Levendorskii, S. L. and Soibelman, Y. S.: Algebras of functions on compact quantum groups, Schubert cells, and quantum tori. Commun. Math. Phys. 139 141-170 (1991)

[21] Lu, J.-H., Weinstein, A.: Poisson Lie groups, dressing transformations and Bruhat decompositions. J. Diff. Geom. 31, 501-526 (1990)

[22] Reshetikhin, N. Yu.: Lectures notes on completely integrable systems and Poisson-Lie groups, Berkeley 1998

[23] Reshetikhin, N. Yu. and Semenov-Tian-Shansky, M.: Quantum R-matrices and factorization problems in quantum groups. J. Geom and Phys. 5, 533-550 (1988)

[24] Semenov-Tian-Shansky, M.: What is a classical $r$-matrix? Funct. Anal. Appl. 17 259-272 (1983)

[25] Semenov-Tian-Shansky, M.: Dressing transformations and Poisson group actions. Publ. RIMS, Kyoto Univ., 21, 1237-1260 (1985)

[26] Soibelman, Y. S.: The algebra of functions on a compact quantum group and its representations. Leningrad Math. J. 2 193-225 (1990)

[27] Yakimov, M.: Symplectic leaves of Poisson homogeneous spaces of complex reductive Poisson-Lie groups. in preparation 